\title{
Horizontal Directional Spectrum Estimation of the Heard Island Transmissions
}

\author{
by Peter Traykovski
}

\author{
B.S. Mechanical Engineering \\ Duke University (1988)
}

Submitted in Partial fulfillment of the requirements for the degree of

OCEAN ENGINEER

at the

MASSACHUSETTS INSTITUTE OF TECHNOLOGY

and

WOODS HOLE OCEANOGRAPHIC INSTITUTION

January (1994)

(C) Peter Traykovski (1994), All rights reserved.

\begin{tabular}{c} 
MARINE \\
BIOLOGICAL \\
LABORATORY \\
\hline LIBRARY \\
\hline $\begin{array}{c}\text { WOODS HOLE, MASS. } \\
\text { W. H. O I. }\end{array}$ \\
\hline
\end{tabular}

The author herby grants to MIT, WHOI and the U.S. Government permission to reproduce and to distribute copies of this thesis document in whole or in part.

Author

Department of Ocean Engineering, Massachusetts Institute of Technology, and the Joint Program in Oceanography and Oceanographic Engineering, Massachusetts Ifistitute of Technology/ Woods Hole Oceanographic Institution

- January 24,1994

Supervised by

Prof. Arthur B. Baggeroer Ford Professor of Engineering, Dept. of Ocean Engineering, and Dept. of Electrical Engineering and Computer Science
Massachusetts Institute of Technology
Thesis Supervisor

Certified by

Prof. Arthur B. Baggeroer

Chairman, Joint Committee for Applied Ocean Science and Engineering Massachusetts Institute of Technology/WWoods Hole Oceanographic Institution

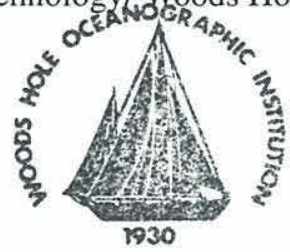





\begin{abstract}
:
In 1991 the Heard Island Feasibility Test demonstrated that it is possible to transmit coded acoustic signals nearly half way around the world. One of the key issues in the feasibility test was to determine the spatial structure of the received transmissions. In this thesis, data from the Canadian Defense Research Establishment Pacific horizontal line array is used to form an estimate of the directional power spectrum. This spectrum determines if any horizontal multipath is detectable. The preliminary signal conditioning, including frequency spectrum estimation and demodulation required before beamforming is described. Conventional and adaptive beamforming methods are examined with synthetic data to demonstrate the limitations on the directional spectrum results. The principle result of this work is that no stable horizontal multipath is evident. The mean arrival angle for the five hours of data analyzed is $212^{\circ} \pm 1.5^{\circ}$.
\end{abstract}





\section{Acknowledgments:}

I wish to thank E. Scheer at WHOI for the use of his signal processing software, and for maintaining the system that was used for this thesis. The Office of Naval Research provided funding for the author under the ONR fellowship program. This project was made possible by horizontal line array data acquired by G. Heard at Defense Research Establishment Pacific, Canada. Lastly I would like to thank my advisor Prof. A. B. Baggeroer for his direction and insight into the spatial signal processing used in this thesis. 



\section{Table of Contents:}

CHAPTER 1 Introduction 9

1.1 Experiment overview 9

1.2 Horizontal multipath 10

1.3 Detecting horizontal multipath 11

1.4 Overall processing flow 12

CHAPTER 2 Preliminary Signal Conditioning 15

2.1 Signals 15

2.2 Records 15

2.5 Decimation and demodulation 16

2.3 Phase coherence 22

CHAPTER 3 Beamforming Methods and Limitations 25

3.1 Array geometry 25

3.2 Conventional processing 26

3.3 Sample covariance matrix formation 30

3.4 Adaptive processing 32

3.5 Array geometry sensitivity 43

CHAPTER 3 Appendix A: Adaptive Processor Graphic Interpretation 51

CHAPTER 4 Directional Spectrum Estimation Results 61

4.1 Conventional medium resolution processing 62

4.2 Conventional high resolution processing 67

4.3 Compensating for array geometry variations 68

4.4 High resolution adaptive processing 74

4.5 Beamforming conclusions 87 



\subsection{Experiment overview}

In January of 1991 the Heard Island Feasibilty Test (HIFT) demonstrated it was possible to transmit phase coded acoustic transmissions over global ranges. The acoustic signals were transmitted from the source ship located at $54^{\circ} \mathrm{S}, 74^{\circ} \mathrm{E}$ in the southern Indian Ocean near Heard Island and were received even at the most distant sites off the east and west coasts of North America (figure 1.1.)

The motivation for the test was that since the propagation speed of acoustic waves is dependent on water temperature the change in the travel time of the signals can be used to detect changes in the ocean temperature averaged over the paths of the signals. One of the central questions in the Feasibility Test was can phase coherent processing be exploited at these ranges? If this was possible it was expected that the travel time resolution required to measure small temperature changes could be achieved (Munk et al.(1993), [1].)

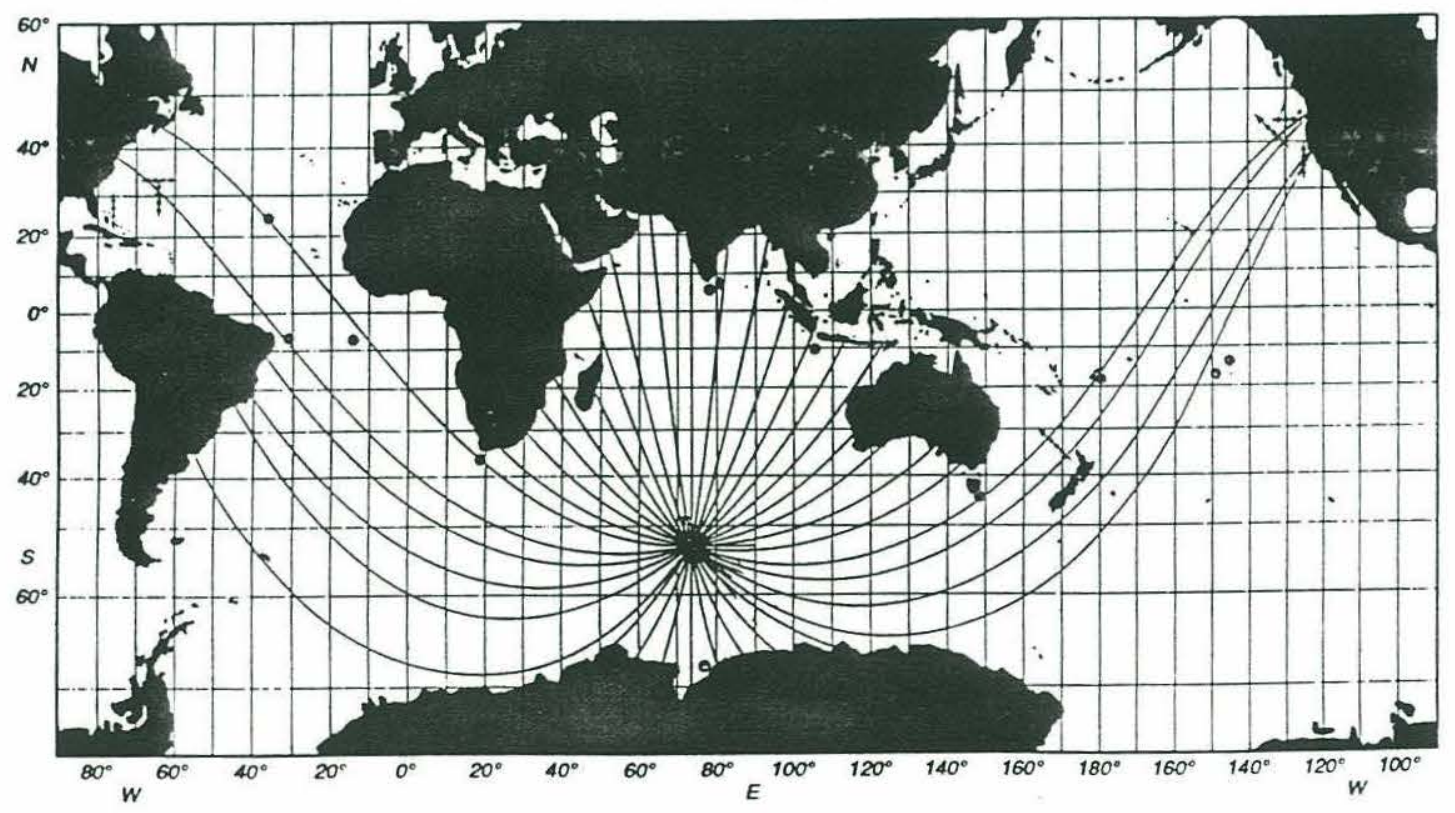




\subsection{Horizontal multipath}

The other key issue of the HIFT was to determine the spatial characteristics and the stability of these characteristics for long range transmissions. The spatial characteristics have a profound impact on travel time estimation. If multiple paths connect the source and receiver, they may have different path lengths and/or travel times. This leads to multiple estimates for arrival time. Figure 1.1 demonstrates a possible scenario where two horizontal multipath connect the source at Heard Island with a receiver off the west coast of North America.

The spatial characteristics of the signal are determined by the dependencies of the sound speed on temperature and pressure which cause sound waves to refract. On the vertical axis this leads to well known waveguide effects such as the formation of rays and modes of propagation in the waveguide. On the horizontal axis refraction causes the signal to deviate from the great circle path which is the shortest path between two points on a spherical earth, and to travel along a refracted geodesic path. The geodesic path differs from the great circle in that the geodesic path accounts for the polar flattening of the earth. The refraction from the geodesic path has been demonstrated experimentally in the analysis of the 1960 Perth-Bermuda transmissions (Heaney et al. (1991),[2]). In this analysis travel times were compared to model results based on horizontally refracted adiabatic modes. The multiple arrival times were explained by two widely separated horizontal multipaths. The implications of multiple arrival times are motivating factors for this work.

Along a geodesic from Heard Island to the west coast of the United States the sound waves encountered strong horizontal temperature gradients, such as those associated with the Antarctic circumpolar current. Variable bathymetry also plays an important role in horizontal refraction as sound waves are refracted away from shallower water. Given the correct refractive conditions the transmissions could have travelled along multiple horizontal paths from Heard Island to the receiving stations. McDonald et al. predicted horizontal multipath consisting of two eigenray bundles reaching the receiving station [3]. The mean predicted horizontal arrival angles for these two bundles were $214.2^{\circ}$ and $219.3^{\circ}$ with respect to true north. The estimated bottom losses for these paths were approximately $-10 \mathrm{~dB}$ and $-50 \mathrm{~dB}$ respectively. Consequently, the path arriving at $219.3^{\circ}$ may have been very difficult to detect if it existed. Within each of these eigenray bundles the individual modes of propagation had horizontal arrival angles differing by a tenth of a degree to half a degree. 


\subsection{Detecting horizontal multipath}

During the HIFT the Canadian Defense Research Establishment Pacific (DREP) Vessel CFAV ENDEAVOUR towed a horizontal array. In this thesis the data from this array is beamformed and an estimate is formed of the angular dependence of the incoming power from the Heard Island source. Both conventional and adaptive beamforming methods are used. The central questions this thesis addresses are:

- Can horizontal mutlipath be detected at a horizontal receiving array?

- What were the arrival angles of the paths?

Multipath with power sufficiently above the noise level to allow detection and separated by a resolvable angle was not detected. Low power level mutlipath may exist. If horizontal multipath exists it could lead to multiple estimates for arrival time and thus difficulty in detecting a climate signal.

Doppler shifts can also be used to estimate launch and arrival angles. In the case of the HIFT the source is moving so if the receiver is stationary only a launch angle can be estimated. If the receiver is also moving both a launch angle and a receiver angle may be estimated. This technique is used with limited success in this thesis. The primary technique of horizontal arrival angle determination is horizontal beamforming.

If the multipath are consistently distinguishable they can be used to generate multiple estimates for travel time trends. Depending on how the multipath sample the oceanographic mesoscale random processes these estimates may be independent. Simple circular arc geometry in figure 1.2 shows that paths separated by $\theta=0.5^{\circ}$ degrees in arrival angle are separated by $\delta \sim 80 \mathrm{~km}$ at half range of $9000 \mathrm{~km}$ and arrivals separated by $\theta=5^{\circ}$ are separated by $\delta \sim 400 \mathrm{~km}$ at half range. While this simple geometric argument ignores the realities of the actual refracted paths it indicates that the paths would encounter different oceanographic conditions and bathymetric features.

FIGURE 1.2

Horizontal Refraction model

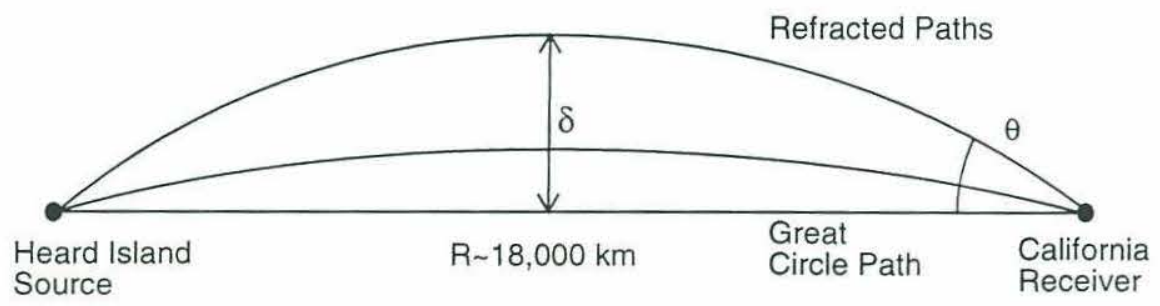




\subsection{Overall processing flow}

The following flow chart shows the sequence of steps in forming an estimate of the angular power spectrum. Appropriate diagrams and descriptions of the individual steps are found in the indicated chapters.

\section{FIGURE 1.3}

Overall Processing Flow Chart

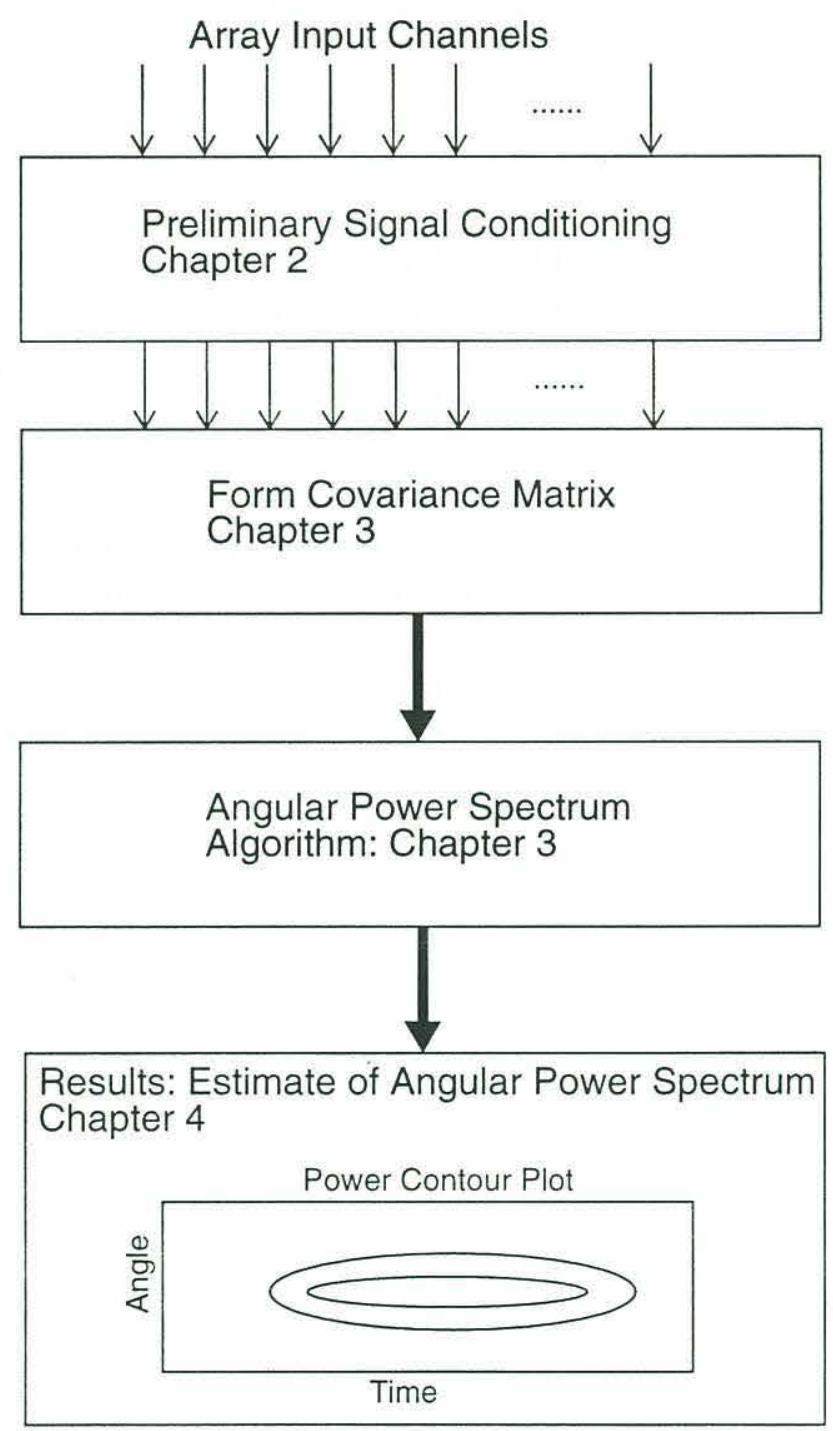


[1] W.H. Munk, R. C. Spindel, A. B. Baggeroer, and T. G. Birdsall (1993), "The Heard Island Feasibilty Test," J. Acoust. Soc. Am. (special issue.)

[2] K.D. Heaney, W. A. Kuperman, and B. E. McDonald (1991), "Perth-Bermuda sound propagation (1960): Adiabatic mode interpretation," J. Acoust. Soc. Am. 90(5), November 1991, p.2586

[3] B. E. McDonald, M. D. Collins, W. A. Kuperman and K. D. Heaney (1993),"Comparison of Data and Model Predictions For Heard Island Acoustic Transmissions," $J$. Acoust. Soc. Am. (HIFT special issue.)

[4]W.H. Munk and Forbes, A.M.G. (1990), "Global Ocean Warming: An Acoustic Measure," J. Phs. Oceanogr: 19, p. 1765-1778 (1989). 
CHAPTER 2

\section{Preliminary Signal Conditioning}

\section{$2.1 \quad$ Signals}

Three types of signals were used in the HIFT. (Munk et al.(1993)[1]) The first type of signal was a simple continuous wave (CW) signal. It is ideal for angular power spectrum estimation since it has the highest signal to noise ratio as all the energy is concentrated in one frequency. The second type of signal was a phase shifted pentaline $(\mathrm{P})$ signal. This signal has five tonal components each spaced $1.9 \mathrm{~Hz}$ apart. This type of signal is used for angular power spectrum estimation since the tonals can be narrow bandpass filtered and the angular power spectrum estimates for different frequency bands can be compared. The third type of signal was a pseudo-random phase shift signal with a bandwidth of $10 \mathrm{~Hz}$. This signal has a impulse-like auto-covariance structure and is ideal for time delay estimation. The carrier frequency was chosen to be $57 \mathrm{~Hz}$. This was low enough so absorption was not a problem on the $18,000 \mathrm{~km}$ path, and the 50 and $60 \mathrm{~Hz}$ power frequencies were avoided. Lower frequencies were not optimum because of increased bottom interaction. The following table summarizes the transmissions used:

TABLE 2.2

\begin{tabular}{llll}
\hline Event Name & $\begin{array}{c}\text { Transmission Time } \\
\text { (dd/hnmm Z) }\end{array}$ & Signal Type & $\begin{array}{l}\text { Receive Time } \\
\text { (Tape Start, dd/hhmm Z) }\end{array}$ \\
\hline Event 15 (E15) & $29 / 1500$ & $\mathrm{P}$ & $29 / 1815$ \\
Event 18 (E18) & $30 / 0000$ & $\mathrm{CW}$ & $30 / 0312$ \\
Event 19 (E19) & $30 / 0300$ & $\mathrm{P}$ & $30 / 0613$ \\
Event 22 (E22) & $30 / 1200$ & $\mathrm{CW}$ & $30 / 1517$ \\
Event 23 (E23) & $30 / 1500$ & $\mathrm{P}$ & $30 / 1813$ \\
& & & \\
\hline
\end{tabular}

\subsection{Records}

After $3 \mathrm{hr} .17 \mathrm{~min}$. acoustic travel times the signals were received by the DREP array, the Canadian Ocean Acoustic Measurement System (COAMS). The array was located in a region approximately $420 \mathrm{~km}$ west of Los Angeles. Figure 2.1 shows the location of each reception superimposed on contours of the angle of declination of the earth's magnetic field.(NOAA chart 18020 [2]) This information is important in chapter 4 because 
the heading sensors measure the array direction with respect to magnetic north and must be referenced to true north. The figure also shows a compass with the predicted arrival direction of $214^{\circ} \mathrm{T}$ (with respect to true north) marked.

\section{FIGURE 2.1}

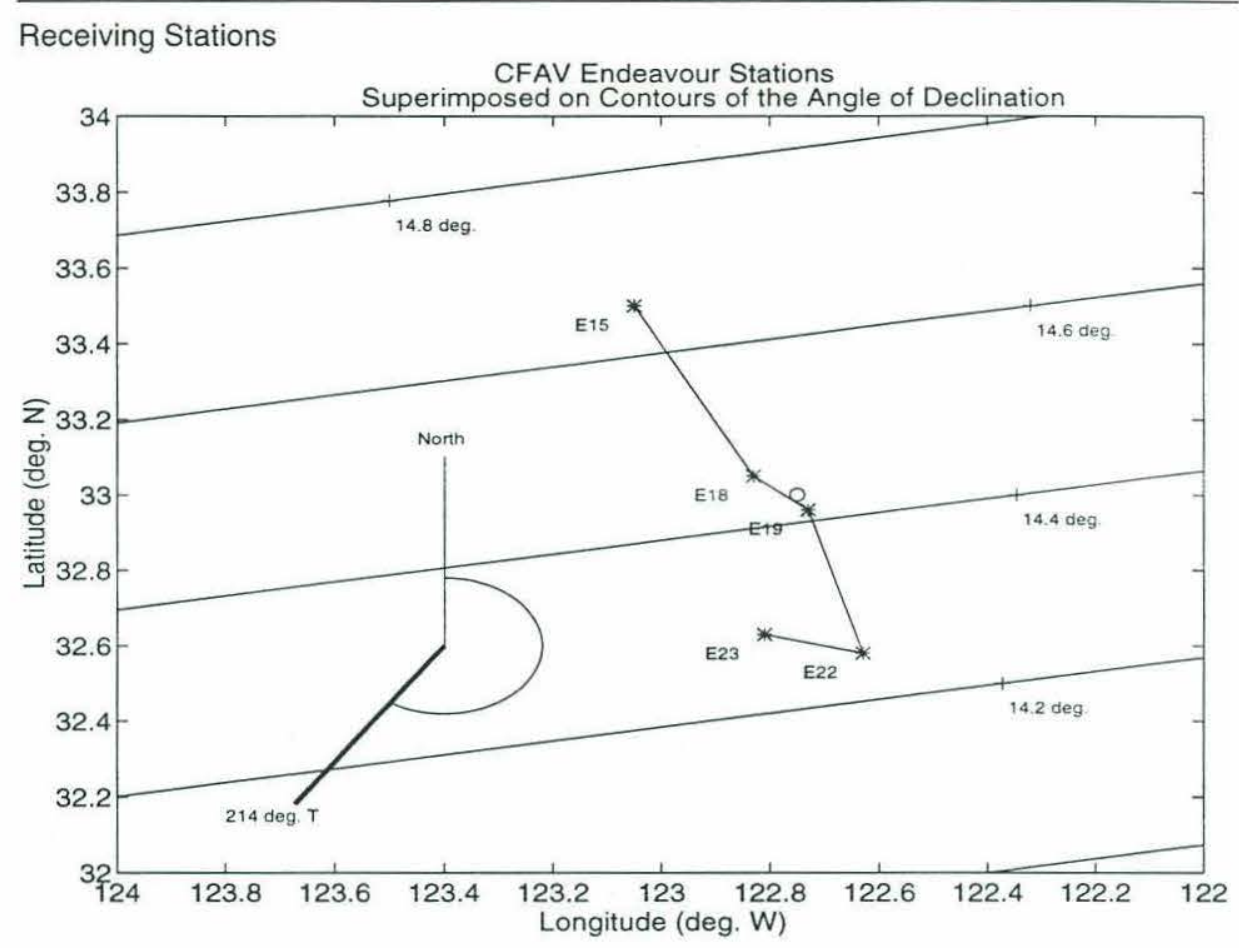

The receiving array contained 128 channels. Digitized data for the five specified transmissions were recorded on $8 \mathrm{~mm}$ tape and sent to the Woods Hole Oceanographic Institution. The time series in each channel are approximately $1 \mathrm{hr}$. $15 \mathrm{~min}$. long, sampled at a frequency of $699.05 \mathrm{~Hz}$. This creates 800 Mbyte data files per event when stored in short integer format. Decimation is required to reduce the data to a more practical size for signal processing.

\subsection{Decimation and demodulation}

The processes of decimation and demodulation occur in two stages. The first stage of demodulation removes the carrier frequency and allows baseband decimation. The next stage demodulates any Doppler shift caused by source and receiver relative motions. This requires spectral analysis to determine the Doppler shift. Figure 2.2 summarizes the preliminary signal conditioning performed separately on each channel. 


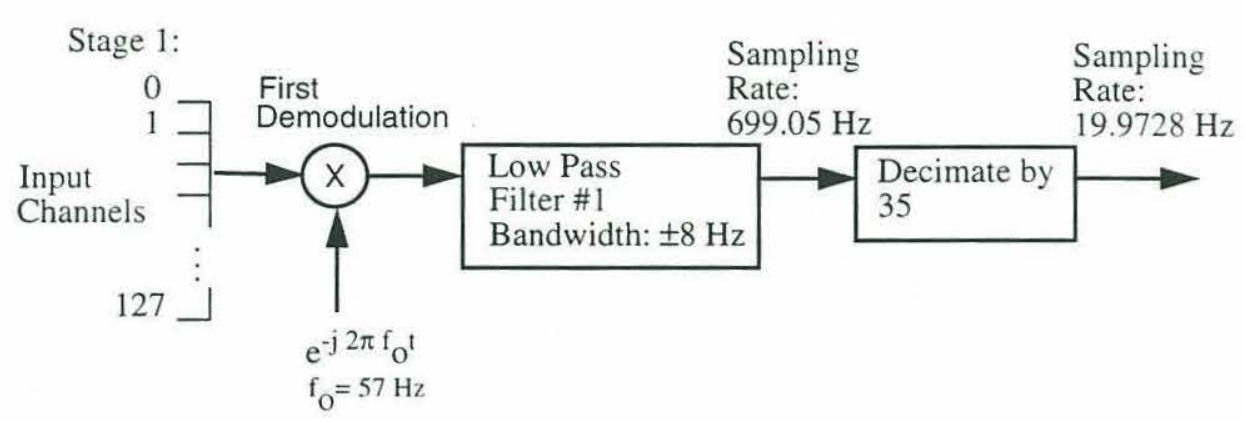

Stage 2:

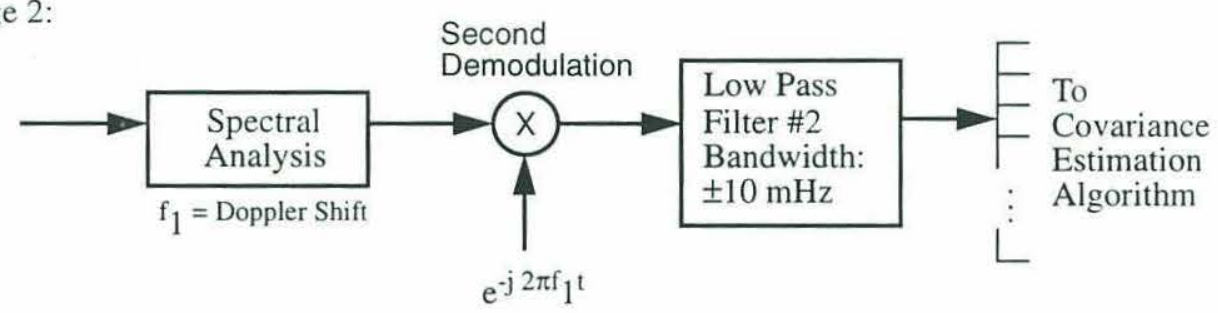

\subsubsection{Stage 1: Decimation}

The signals are demodulated to remove the $57 \mathrm{~Hz}$ carrier frequency. This creates a complex signal centered at $0 \mathrm{~Hz}$ plus any Doppler frequency shift. A low pass filter (lpf. \#1) designed using the Parks-McClellan algorithm with a passband of $\pm 8 \mathrm{~Hz}$, stop band starting at $9.5 \mathrm{~Hz}$, and rejection of $62 \mathrm{~dB}$ is used to remove frequency components greater than the Nyquist for decimation. The frequency response $\mathrm{H}(\mathrm{f})$ for this filter is shown in figure 2.3. Since the widest band signal that is analyzed is the pentaline with a bandwidth of $1.9 \mathrm{~Hz} \times 4= \pm 3.8 \mathrm{~Hz}$ a decimation factor of 35 is chosen. The sampling rate is reduced from 699.05 to $19.9728 \mathrm{~Hz}$ by decimation. The effective Nyquist frequency is now 9.986 which adequately represents the highest components of the pentaline signal. The decimation uses the overlap-save method to accommodate the large data block size. In figure 2.3 the unwrapped phase for low pass filter \#1 is displayed. The group delay in the pass band is given by:

$$
\mathrm{T}_{\mathrm{E}}=\frac{1}{2 \pi} \frac{\mathrm{d} \phi}{\mathrm{df}}=2.14 \mathrm{~s},
$$

which is half of the filter impulse response length of $4.29 \mathrm{~s}$ as expected. The actual implementation of the processing is programmed in the C-language code dem.c (E. Scheer [3]) with modifications to cancel the filter delay. 


\section{FIGURE 2.5}

FIGURE 2.6

\section{Filter Frequency Response}
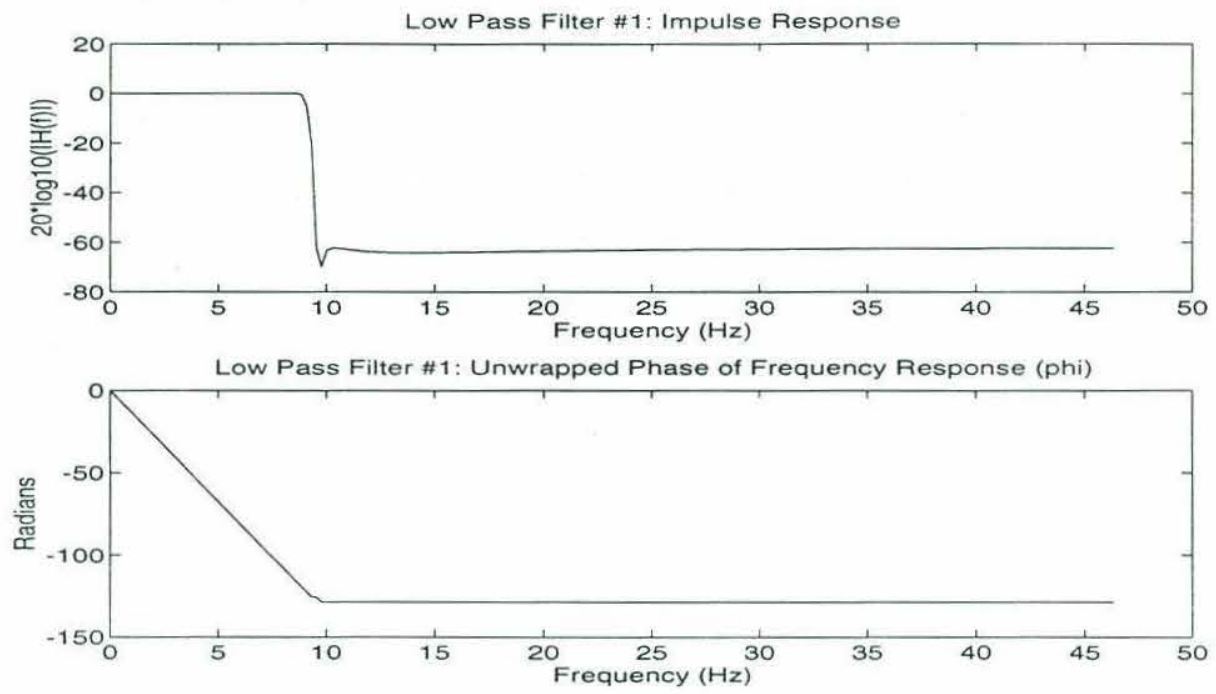

\subsubsection{Spectral analysis}

Once the data is decimated and demodulated to remove the carrier the relative power in the frequency components are examined vs. time to resolve the Doppler shift. As shown in figure 2.4 conventional methods with a sin squared time window are used to estimate the relative power in the spectral components on one channel. A channel towards the end of the array farthest from the tow ship is used to minimize effects of ship noise.

\section{Spectral Estimation Algorithm}

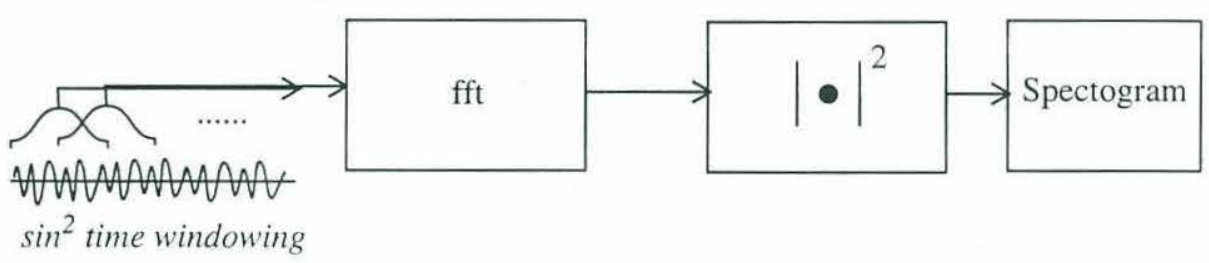

Time windows are 410 seconds long with half window overlaps. The frequency resolution for this time window length is approximately $(1 / 410 \mathrm{sec}$. $)=2.5 \mathrm{mHz}$. No averaging is used in the spectral estimation algorithm. Using a definition of signal to noise ratio (SNR) of

$$
\text { SNR }=10 \log _{10}\left(\frac{\int \Psi_{\mathrm{s}}(\mathrm{f})|\mathrm{H}(\mathrm{f})|^{2} \mathrm{df}}{\int \Psi_{\mathrm{n}}(\mathrm{f})|\mathrm{H}(\mathrm{f})|^{2} \mathrm{df}}\right)
$$

where $\Psi_{\mathrm{s}}$ is the power spectral density of the signal, $\Psi_{\mathrm{n}}$ is the power spectral density of the noise, and $\mathrm{H}(\mathrm{f})$ is the frequency response of each frequency bin in the fft operation, the gain in SNR for a tonal component vs. a white noise process is determined for the spectogram algorithm. This gives $10 \log _{10}(1 / .0025 \mathrm{~Hz})=26 \mathrm{~dB}$ of gain vs. white noise in 
each frequency bin which for most of the records is enough to resolve the signal from the noise. This is adequate to resolve the Doppler shift which is typically 30 to $70 \mathrm{mHz}$. A spectogram for event 18 is shown in figure 2.5.

FIGURE 2.7

\subsubsection{Features of spectogram}

- The signal length on the spectogram is one hour long beginning roughly two minutes into the recorded data. This corresponds to a $3 \mathrm{hr}$. $17 \mathrm{~min}$. travel time.

- The noise floor is 15 to $18 \mathrm{~dB}$ down from the peak signal level. With the $26 \mathrm{~dB}$ processing gain this corresponds to a SNR of approximately -8 to $-11 \mathrm{~dB}$ on one hydrophone. This agrees with the results of G. Heard shown in table 2.2 (Heard and Chapman, (1993)[4]).

- The magnitude of the received signal fluctuates by $15 \mathrm{~dB}$ in four major arrival groups. The temporal distribution of the magnitude fluctuations is not consistent between events, but the fluctuations are consistently observable. The exact reasons for these fluctuations are not known.

- The mean Doppler shift of $-23 \mathrm{mHz}$ is clearly visible on the spectogram. Since the signal has been demodulated to remove the carrier frequency the Doppler shift is measured relative to $0 \mathrm{mHz}$. There are some fluctuations about this mean due to variations in the relative velocities of the source and receiver, but these are within \pm 10 $\mathrm{mHz}$ of the mean. 


\subsubsection{Doppler analysis}

Spectograms for the other events were also plotted to resolve Doppler shifts. The following table summarizes the Doppler shifts for each event:

TABLE 2.2

\begin{tabular}{|c|c|c|c|c|c|}
\hline Event Name & $\begin{array}{c}\text { Predicted Source } \\
\text { only Doppler } \\
(\mathrm{mHz})\end{array}$ & $\begin{array}{c}\text { Predicted Total } \\
\text { Doppler } \\
(\mathrm{mHz})\end{array}$ & $\begin{array}{c}\text { Predicted Total } \\
\text { Upper Bound } \\
\text { Lower Bound } \\
(\mathrm{mHz})\end{array}$ & $\begin{array}{c}\text { Measured Doppler } \\
\text { from Spectogram } \\
(\mathrm{mHz})\end{array}$ & $\begin{array}{c}\text { Input SNR } \\
(\text { Heard[4]) } \\
(\mathrm{dB})\end{array}$ \\
\hline \hline Event15 & 48 & 88 & $\begin{array}{c}103 \\
74\end{array}$ & 71 & -14.6 \\
\hline Event18 & -51 & -20 & -4 & -23 & -10.4 \\
\hline Event19 & -59 & -22 & -34 & -35 & -15.1 \\
\hline Event22 & -56 & -41 & -26 & -65 & -16.3 \\
\hline Event23 & -41 & -15 & -52 & -36 & -28 \\
\hline
\end{tabular}

The total predicted Doppler shift is computed using a signal arrival angle of $212^{\circ} \mathrm{T}$ from the horizontal beamforming results of chapter 4 , and a launch angle of $135^{\circ} \mathrm{T}$. The launch angle is determined from Doppler shifts with a moving source and a stationary receiving array located off of Monterey (Sperry, (1994)[5].) This launch angle gives excellent agreement between predicted and measured Doppler shifts on the Monterey array. The velocities of both source and receivers are taken from ships' logs of the R/V CORY CHOUEST and CFAV ENDEAVOUR respectively. The following equation gives the total predicted Doppler shift for the towed horizontal array where both the source and receiver are moving:

FIGURE 2.8

$$
\begin{gathered}
\Delta \mathrm{f}=\frac{\mathrm{f}}{\mathrm{C}} \cdot\left(\mathrm{V}_{\mathrm{s}} \cdot \cos \theta_{\mathrm{s}}+\mathrm{V}_{\mathrm{r}} \cdot \cos \theta_{\mathrm{r}}\right) \\
\Delta \mathrm{f}=\text { Doppler Shift, } \mathrm{C}=\text { Sound Speed, } \mathrm{f}=\text { Carrier Frequency }
\end{gathered}
$$

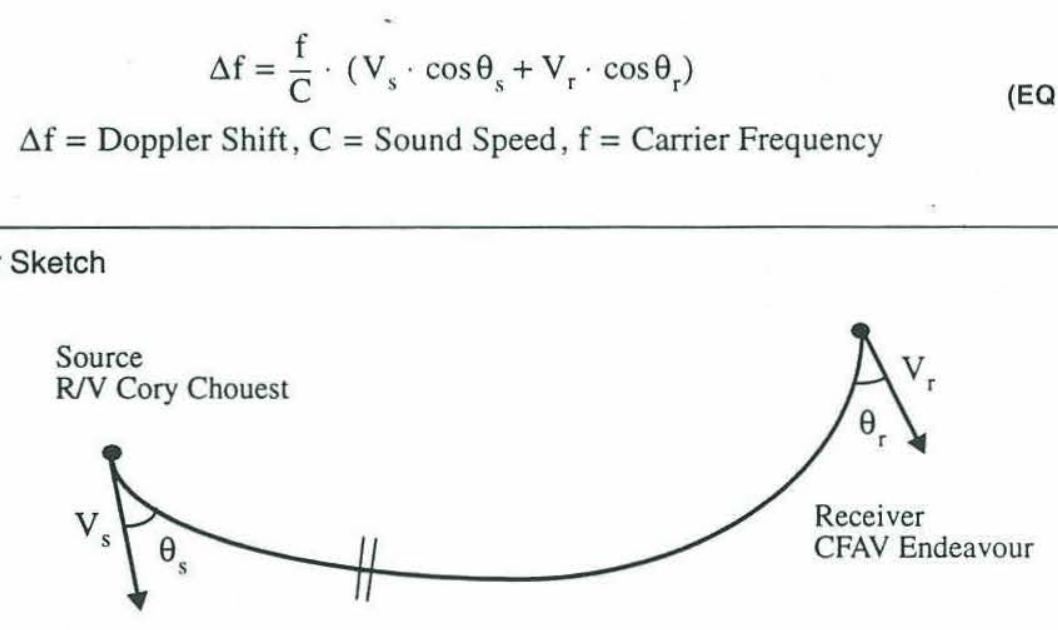

Doppler Sketch

As shown in table 2.2 the measured and total predicted Doppler shifts are not in very good agreement. An error in any of the four variables shown in figure 2.6 could cause a 
difference. The source velocity and angle are assumed to be fairly accurate due to the fact that Doppler launch angle estimation with stationary receivers is accurate. The Doppler shifts due to source motions only are displayed in the second column of table 2.2 .

The primary reason for the difference between the measured and predicted Doppler shifts is uncertainty in source and receiver relative motions. Part of this uncertainty is due to array deformation. The receiver angle $\left(\theta_{\mathrm{r}}\right.$ in figure 2.6) is the difference between the arrival angle of the signal and the angle of the receiving elements velocity vector. Although the arrival angle $\left(212^{\circ} \mathrm{T}\right)$ is well known from the beamforming results of chapter 4 , the velocity of the receiving element may be inaccurate in both magnitude and direction. The alignment of the array is used as the receiving elements direction, but this may not be the actual direction of the receiving element. Estimated errors of up to \pm 0.5 knots in speed or \pm 10 degrees in direction are used to determine the upper and lower bound for the predicted total Doppler shift. The error only effects the predicted Doppler shift due to receiver motions since the source Doppler shift is accurate. The measured Doppler is only within the error bounds for events 18 and 19, suggesting the estimates for velocity errors are low. On the other events the measured Doppler is almost within the error bounds, but errors of \pm 20 degrees in direction and \pm 1 knot in speed are required to encompass the measured results within the error bounds of the predicted results. When the amount of curvature and rate of turning of the array are examined in detail in chapter 4 , it is shown that for events 18 and 19 the array is closest to linear.

A secondary reason for the difference between measured and predicted Doppler shifts is the measured shift may not be entirely accurate due to low SNR. The low SNR requires a long time window to resolve the mean Doppler shift. Since the mean Doppler shift is resolved clearly on the spectograms with a long enough time window, this is used to determine the demodulation frequency rather than using the predicted Doppler shifts.

\subsubsection{Second demodulation}

The Doppler shift for each event is removed by a second demodulation. The signal is then filtered with a very narrow band filter (lpf\#2 in figure 2.2) to create a narrow band signal for beamforming and to remove noise in other irrelevant frequency bands. The filter is designed using the Parks-McCellan algorithm. It has a pass band cutoff at $\pm 10 \mathrm{mHz}$ and a stop band beginning at $\pm 20 \mathrm{mHz}$ with a rejection of $69 \mathrm{~dB}$. The frequency response is shown in figure 2.7. This filter is designed to be as narrow as possible without filtering any of the desired signal. The group delay of this filter is half the filter length as is case with low pass filter \#1. This delay is cancelled by a time index shift in the processing code dem.c.

As mentioned previously the Doppler shift is not constant over the one hour event duration, but fluctuates slightly due to changes in transmitter velocity. A heading sensor on the receiving array changes its alignment up to 8 degrees over the course of one hour. If 
the most sensitive case (in terms of a change in Doppler shift due a change in course) of $\theta_{\mathrm{r}}$ close to $90^{\circ}$ occurs the Doppler shift is $\pm 10 \mathrm{mHz}$. for a $10^{\circ}$ direction change. This is calculated with source and receiver speeds both equal to 3 knots. Thus the fluctuations in Doppler shift remain within the bounds of the low pass filter passband of $\pm 10 \mathrm{mHz}$. With the low SNR on one hydrophone, a very long (approximately $1000 \mathrm{sec} .+$ ) time window is required to resolve the signal clearly enough to track Doppler fluctuations around their one hour mean shift. The time scale of the fluctuations are less than this window length thus making this method inappropriate. As seen on the spectogram the mean shift is clearly identified, and within the observational limits it did not appear to fluctuate beyond the bandwidth of the low pass filter used.

\section{FIGURE 2.9}

\section{Filter Frequency Response}
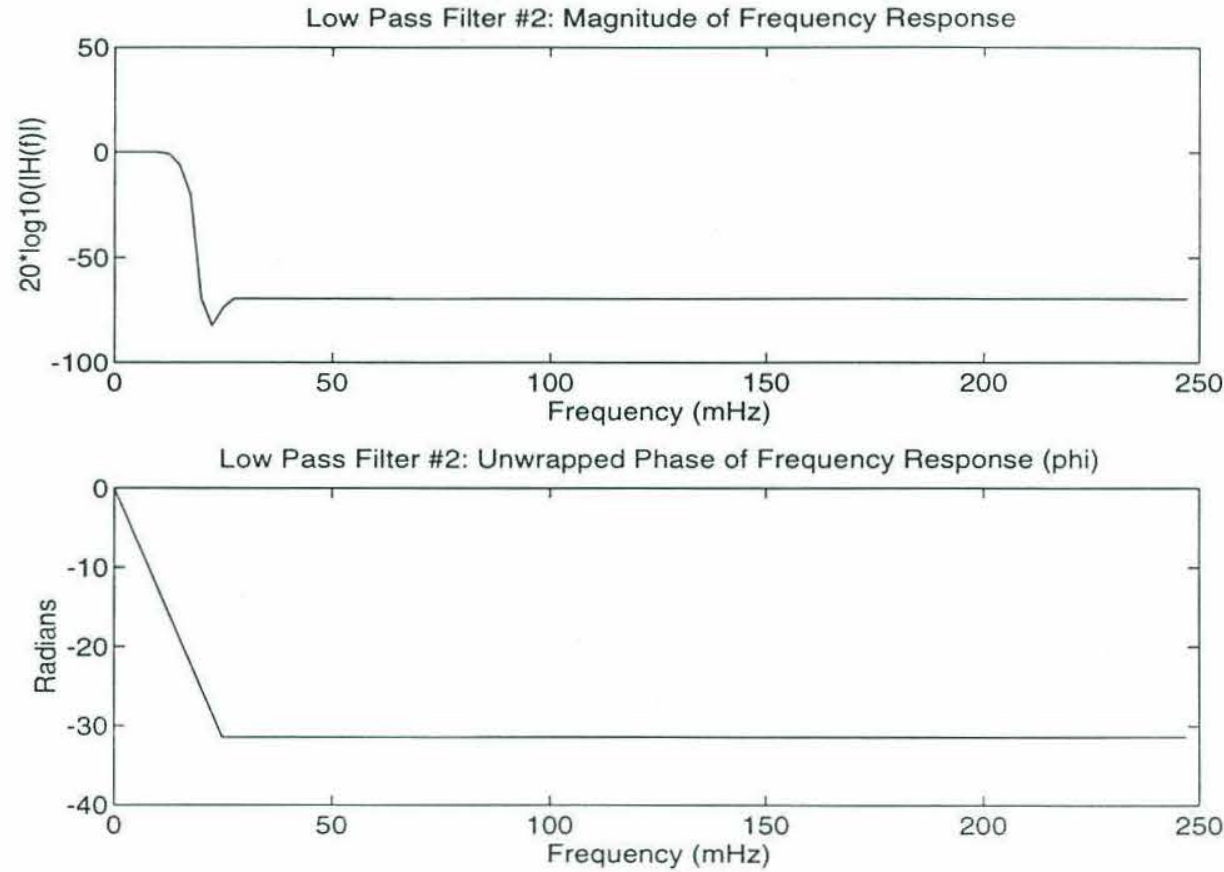

\subsection{Phase coherence}

Since beamforming operations rely on a phase delay between elements of the array a certain degree of phase coherence is required along the array. One simple way to investigate this before beginning beamforming operations is to multiply one channel times the complex conjugate of an adjacent channel. As seen in equation 2.3 this causes the carrier frequency time dependence $\left(\mathrm{f}_{0} \mathrm{t}\right)$ to cancel out and only leave a constant $\left(\mathrm{T}_{72^{-}}\right.$ $\mathrm{T}_{64}$ ) due to inter element delay in the phase term. The time dependent amplitude of the received signal for channel $n$ is denoted $A_{n}(t)$.

$$
A_{64}(t) e^{-j 2 \pi f_{11}\left(t+T_{64}\right)} \cdot A_{72}(t) e^{j 2 \pi f_{0}\left(t+T_{72}\right)}=A_{64}(t) \cdot A_{72}(t) e^{j f_{10} 2 \pi\left(T_{72}-T_{64}\right)}
$$


The magnitude and phase of this operation for channels 64 and 72 of event 18 are plotted in figure 2.8 .
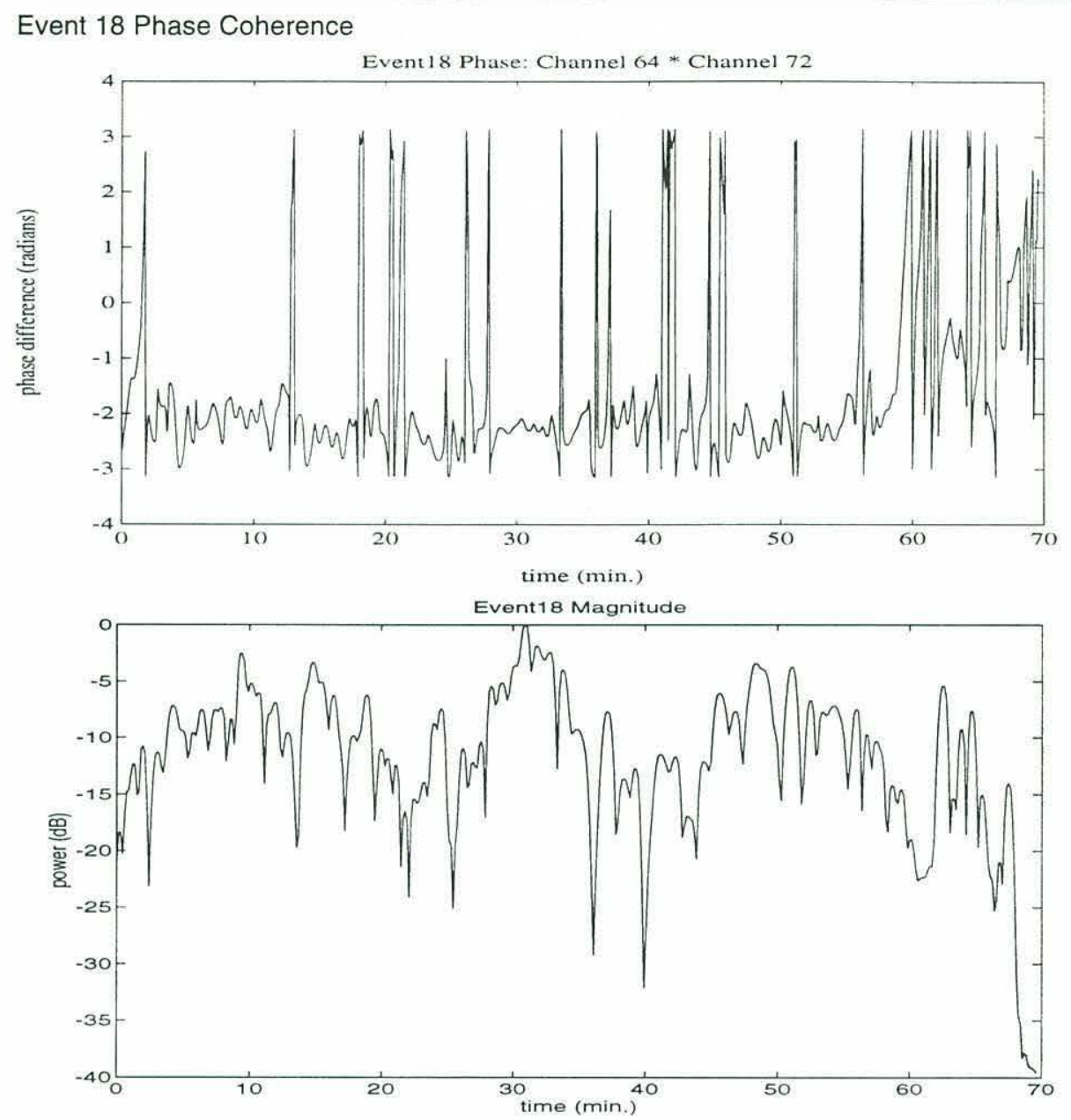

The phase difference between the two channels has some variance about its mean, but the mean is relatively constant at approximately 2.2 radians from $3 \mathrm{~min}$. to $57 \mathrm{~min}$. The variance is due to noise and its effects should be reduced when more than two channels are used. The regions where the phase is not relatively stable are often due to low signal power as seen in the plot of signal power vs. time. The $\mathrm{dB}$ scale for the signal power is normalized so that the maximum power is $0 \mathrm{~dB}$. 
[1] W.H. Munk, R. C. Spindel, A. B. Baggeroer, and T. G. Birdsall (1993), "The Heard Island Feasibility Test," J. Acoust. Soc. Am. (special issue.)

[2] U.S. Dept. of Commerce, NOAA. Nat. Ocean Service Chart 18020, San Diego to Cape Medocino (1987).

[3] Eddie Scheer, Woods Hole Oceanographic Institution, Personal Communication.

[4] G. J. Heard and N. R. Chapman (1992). "The Heard Island Feasibility trial: Analysis of Pacific path data obtained with a horizontal line array," J. Acoust. Soc. Am. (special issue.)

[5] Sperry, B. (1993) "Masters Thesis on Vertical Mode Structure" 


\section{CHAPTER 3}

\section{Beamforming Methods and Limitations}

In this chapter the beamforming methods used to estimate the angular power spectrum of the data received on the COAMS array during the HIFT are discussed. This is not intended to be a general discussion of beamforming techniques, but is intended to provide motivation for the processing techniques that are used. The interpretations of the beamformers presented in this chapter help in understanding the results and the limitations on the results of chapter 4 .

\subsection{Array geometry[1]}

The geometry of the COAMS array is used to generate synthetic data. The spatial sampling as determined by the array geometry determines many of the characteristics of the angular power estimation results.

\section{FIGURE 3.1}

\section{Array Geometry: Side View}

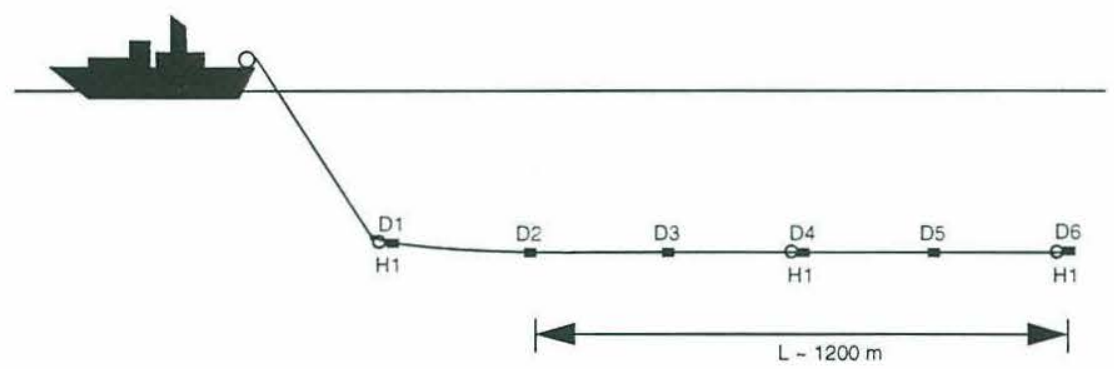

With the nested subarray architecture of the COAMS array it is possible to form many different arrays. The following table summarizes the inter-element spacing, the number of elements $\mathrm{N}$, and the length $\mathrm{L}$ of three different arrays that are used for conventional and adaptive array processing. 


\begin{tabular}{|c|c|c|c|}
\hline Array Name & $\begin{array}{c}\text { Inter- } \\
\text { element } \\
\text { distance } \Delta \mathrm{x}\end{array}$ & $\begin{array}{c}\text { Number of } \\
\text { elements } \\
\mathrm{N}\end{array}$ & $\begin{array}{c}\text { Length } \\
\text { L }\end{array}$ \\
\hline \hline $\begin{array}{c}\text { Conventional Medium Resolu- } \\
\text { tion Array (CMRA) }\end{array}$ & $9.525 \mathrm{~m}$. & 32 & $600.1 \mathrm{~m}$. \\
\hline $\begin{array}{c}\text { Conventional High Resolution } \\
\text { Array (CHRA) }\end{array}$ & $38.1 \mathrm{~m}$. & 32 & $1181.1 \mathrm{~m}$. \\
\hline $\begin{array}{c}\text { Adaptive High Resolution } \\
\text { Array (AHRA) }\end{array}$ & $152.4 \mathrm{~m}$. & 8 & $1066.8 \mathrm{~m}$. \\
\hline
\end{tabular}

The signals at the 8 elements of the AHRA are formed by summing signals from subarrays within the CHRA array (section 3.3.1.3). As shown in figure 3.1 the depth sensors display a upward tilt on the forward section of the array so this part of the array is not used for the beamforming operations.

\section{FIGURE 3.2}

Array Geometry: Top View

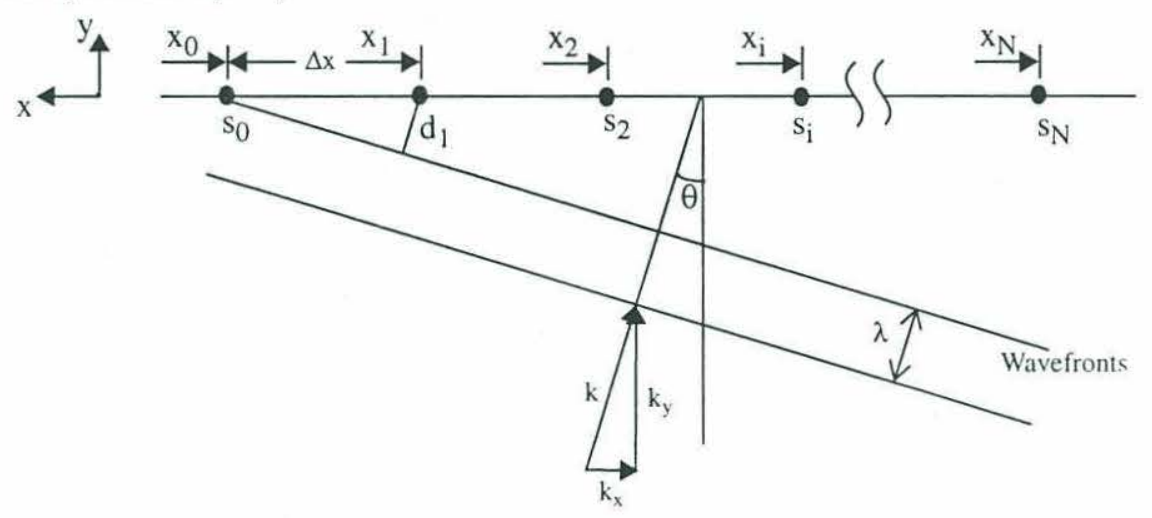

\subsection{Conventional processing}

The signal received at the ith element of the array is denoted as $\mathrm{s}_{\mathrm{i}}$. For the synthetic plane wave model as shown in figure $3.2 \mathrm{~s}_{\mathrm{i}}$ has the form

$$
S_{i}=e^{j \omega\left(t-\frac{d_{1}}{C}\right)}=e^{j \omega\left(t-\frac{x_{1} \cdot \sin (\theta)}{C}\right)}
$$

where $d_{i}$ is the distance from the ith element to the wavefront intersecting the zeroth element, and $\mathrm{C}$ is the phase propagation speed of the wave. 
Demodulation removes the carrier time dependence. Using the definition of wavenumber $\mathrm{k}=\frac{\omega}{\mathrm{C}}$, and its projection along the array axis $\mathrm{k}_{\mathrm{x}}=\mathrm{k} \cdot \sin \theta, \mathrm{s}_{\mathrm{i}}$ is written as: $s_{i}=e^{j k_{x} x_{1}}$. A column vector containing wavenumber representations of the received signals is denoted as $\mathbf{S}$ and for the synthetic plane wave model it has the form:

$$
s=\left[\begin{array}{c}
e^{j k_{x} x_{1}} \\
e^{j k_{x} x_{2}} \\
e^{j k_{x} x_{1}} \\
\cdots \\
j k_{x} x_{N}
\end{array}\right]
$$

In conventional beamforming a steering vector is chosen so that the phases cancel when the wavenumber projection of the steering vector on the array axis $\left(k_{x}^{d}\right)$ matches the wavenumber projection on the array axis $\left(\mathrm{k}_{\mathrm{x}}\right)$ of the incoming plane wave. Thus the ith element of the steering vector $d(\theta)$ is denoted $d_{i}(\theta)$ and has the form $d_{i}=e^{j k_{x}^{d} x_{1}}$. Conventional beamforming can be written in terms of a normalized inner product $\mathrm{N}^{-1}\left(\mathbf{d}^{\mathrm{H}} \mathbf{s}\right)$ where $\mathbf{d}^{\mathrm{H}}$ is the complex conjugate (Hermitian) transpose of the column vector $\mathbf{d}$ and $\mathrm{N}$ is the number of array elements.

An estimate of the power from a given direction is given by the magnitude squared of the inner product:

$$
\hat{\mathrm{P}}(\theta)=\left|\frac{\mathrm{d}^{\mathrm{H}} \mathbf{s}}{\mathrm{N}}\right|^{2}=\frac{\mathrm{d}^{\mathrm{H}} \mathbf{s s}^{\mathrm{H}} \mathbf{d}}{\mathrm{N}^{2}} .
$$

The outer product $\hat{\mathbf{K}}=\mathbf{s s}^{\mathrm{H}}$ in the power expression is the sample covariance matrix, and is discussed in section 3.4 on adaptive processing methods. ${ }^{1}$

For a linear equally spaced array the inner product operation $\mathbf{d}^{\mathrm{H}} \mathbf{s}$ forms a discrete fourier transform. If the elements of $\mathbf{d}$ are uniformly weighted, the output of the inner product in response to a plane wave with $\mathrm{k}_{\mathrm{x}}=0$ results in a sinc function as the steering vector sweeps through the range of possible steering wavenumbers. The denominator of the sinc function in equation 3.4 controls the spatial aliasing rate.

$$
\hat{\mathrm{P}}(\theta)=\frac{\operatorname{sinc}\left(\mathrm{k}_{\mathrm{x}} \cdot \frac{\mathrm{L}}{2}\right)}{\operatorname{sinc}\left(\mathrm{k}_{\mathrm{x}} \cdot \frac{\Delta \mathrm{x}}{2}\right)}
$$

If the element spacing $(\Delta x)$ is greater than half the wavelength $(\lambda / 2=13 \mathrm{~m})$ at $57 \mathrm{~Hz}$, spatial aliasing may cause an aliased main lobe to appear in the angle space of the beam

1. Lower case bold letters are used for vectors and upper case bold letters are used for matrices. 
pattern. This occurs with both the high resolution arrays, as shown in figure 3.4. The aliasing lobes are $43^{\circ}$ from the main lobe in the CHRA. This is usually not a problem since the signal must be in a direction roughly towards Heard Island. A possible problem could occur if there is a equally powerful source at $57 \mathrm{~Hz}$ located in an aliased main lobe. The CMRA is designed with $\Delta x$ sufficiently less than $\lambda / 2$ so the aliased main lobe is not in the angle space of the beamformer. This array is used for an initial examination of the angular distribution of the signal power. It is also used to determine if secondary sources could present a problem for the aliased higher resolution arrays. A plot of the entire angle space of the CMRA with a Hamming taper is displayed in figure 3.3. The signal in this plot is a synthetic plane wave from $212^{\circ}$. The second main lobe in this figure is not due to aliasing, but to the symmetry of the array response about its axis.

\section{FIGURE 3.3}

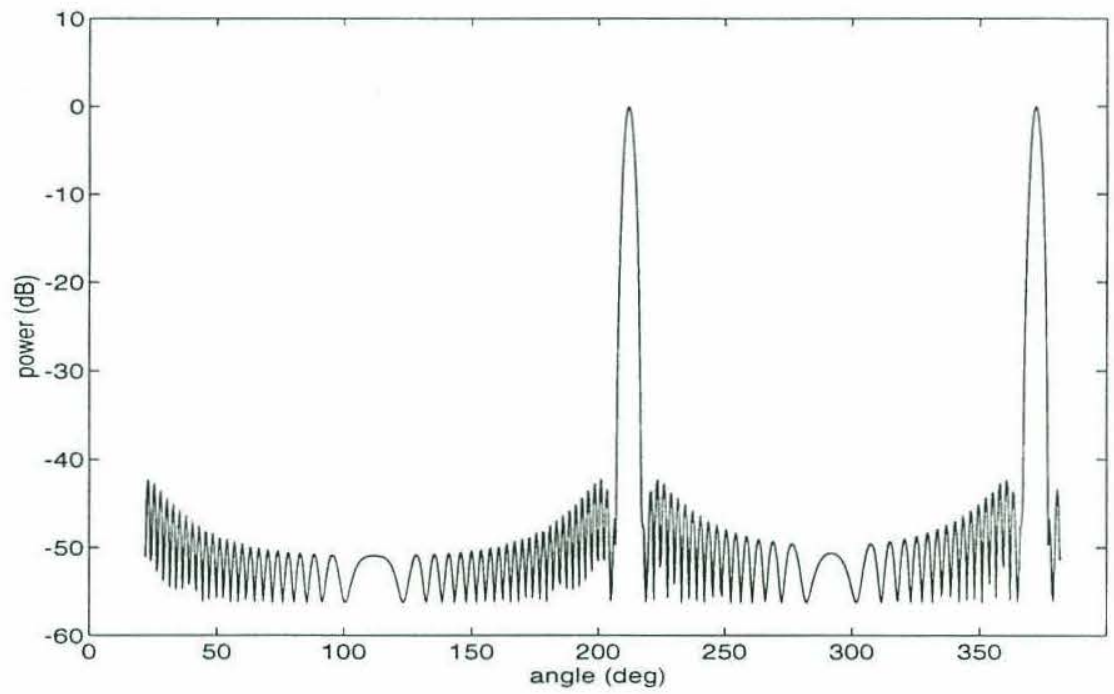

Many different weights for the steering vector are available in the literature (Harris (1978)[2]) to give better sidelobe properties at the expense of a lower angular resolution. The Hamming taper is used in conventional processing. The form of the Hamming weights applied to each element at location $x_{i}$ is:

$$
\mathrm{W}\left(\mathrm{x}_{\mathrm{i}}\right)=0.54-0.46 \cdot \cos \left(2 \pi \frac{\mathrm{x}_{\mathrm{i}}}{\mathrm{L}}\right) \quad \mathrm{x}_{\mathrm{i}}=0, \mathrm{i} \Delta \mathrm{x}, \ldots \ldots, \mathrm{L}
$$

Figure 3.4 shows the response of the $\mathrm{CHRA}$ to a plane wave at $\theta=10^{\circ}$ with respect to broadside $\left(\theta=212^{\circ} \mathrm{T}\right.$ in the absolute coordinate system) for both the uniformly weighted (rectangular) and Hamming taper. Note the highest sidelobes of $-13.7 \mathrm{~dB}$ and $-41.8 \mathrm{~dB}$ respectively. The resolution, defined by the $-3 \mathrm{~dB}$ points on the main lobe, for the uniform taper is $1.1^{\circ}$ while the Hamming taper only achieves $1.6^{\circ}$. With the shorter CMRA, the Hamming taper has a resolution of $3.23^{\circ}$. Because the Hamming taper has lower sidelobes it is used for an initial determination of the direction 
of the signal, while the uniform taper with its higher resolution is used for discriminating multipath signals.

In figures $3.3,3.4$, and in all other beampatterns using synthetic data the angle $202^{\circ}$ is broadside to the array. In analyzing the data recorded off the array the beamformer power output is plotted vs. angle with respect to true north. The received signals have a maximum angle of $40^{\circ}$ with respect to broadside. As seen in figure 3.4, endfire effects are not significant at these angles.

FIGURE 3.4

CHRA Beampattern

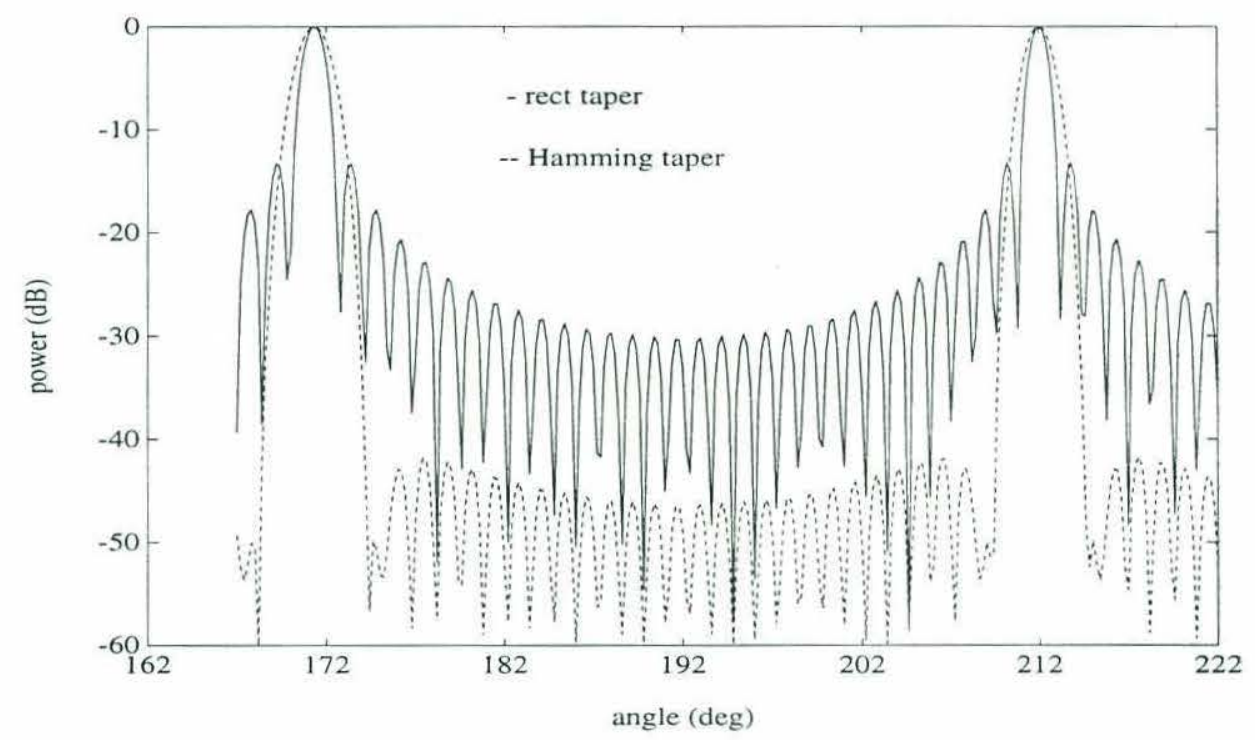




\subsubsection{Multiple plane wave resolution}

One of the key issues in evaluating array performance in detecting multipath is to determine how close can two plane waves be together in angle and still be resolved from each other. This type of performance is evaluated with both taper functions on the CHRA. Figure 3.5 shows how close two plane waves can be while maintaining a $-6 \mathrm{~dB}$ minima between the two peaks to resolve the separate signals. The two plane waves have variable relative power. As the relative power difference increases, the signals must be further apart in angle to be resolved. Table 3.1 summarizes the minimum signal separations for the two plane waves.

TABLE 3.2

Minimum Signal Angular Separations using the CHRA

\begin{tabular}{|c|c|c|c|}
\hline Relative Power & $0 \mathrm{~dB}$ & $-6 \mathrm{~dB}$ & $-12 \mathrm{~dB}$ \\
\hline \hline Uniform Taper & $1.55^{\circ}$ & $1.6^{\circ}$ & $?$ \\
\hline Hamming Taper & $2.5^{\circ}$ & $2.8^{\circ}$ & $3.4^{\circ}$ \\
\hline
\end{tabular}

If the signals differ by $12 \mathrm{~dB}$ or greater it is difficult to distinguish the lower power signal from a sidelobe of the higher power signal with a uniform taper. These minimum signal distances can be reduced by using adaptive beamforming techniques.

FIGURE 3.5

\section{Plane Wave Resolution}

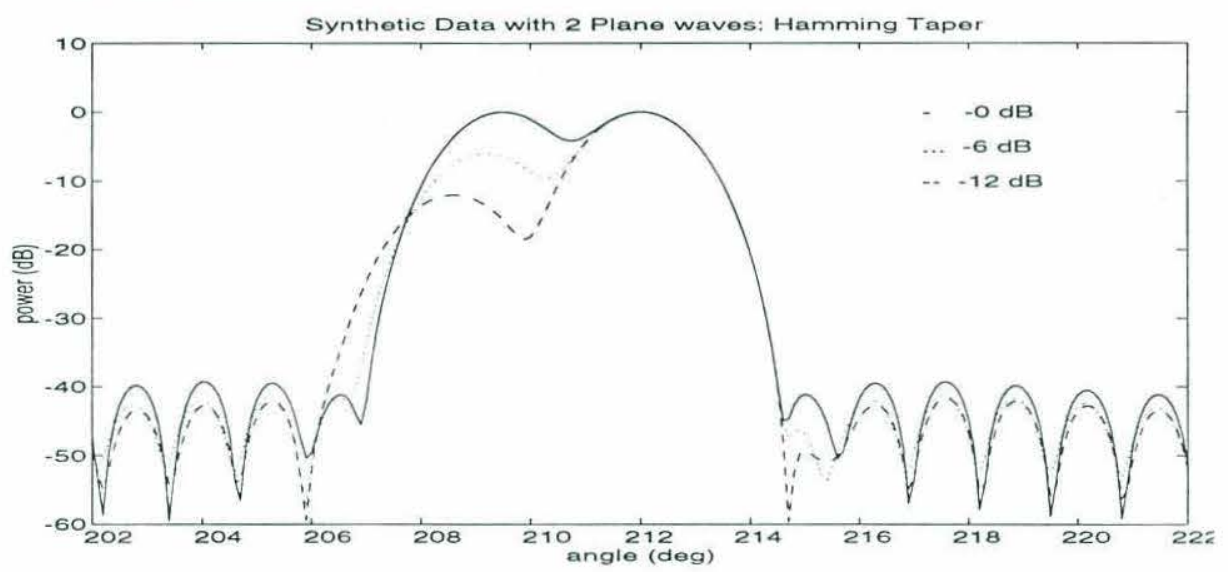

\subsection{Sample covariance matrix formation}

In the previous section the sample covariance matrix was mentioned briefly and its form was described in terms of a wavenumber space representation of a plane wave. The data is received as a time series from each channel so a matrix outer product operation is required to form the sample covariance matrix. 
Input: The inputs to the matrix formation process are $\mathrm{N}$ channels, each containing a discrete time sequence $s_{n}\left(f_{s} t\right)$ sampled at $f_{s}=19.9728$ samples/second from the output of the preliminary signal conditioning process. In order for the time sequence index $\left(\mathrm{f}_{\mathrm{s}} \mathrm{t}\right)$ to be an integer the time ( $t$ ) should be chosen to be an integer multiple of $1 / f_{s}$.

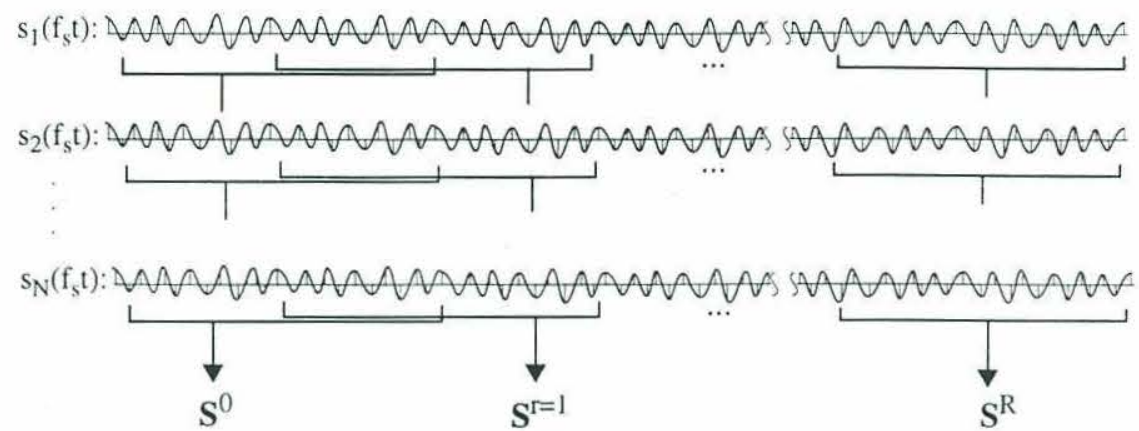

Windowing: The data sequences are time windowed with $\mathrm{R}$ half overlapping, uniformly weighted windows of length $T$ seconds. The windowed data from the rth time window is contained in the matrix $\mathbf{S}^{r}$. The time window index $r$ is an integer counting from 0 to R. Each matrix $\mathbf{S}^{\mathrm{r}}$ can be thought of as a column vector, with each element of the column vector containing a row vector which represents a windowed time series.

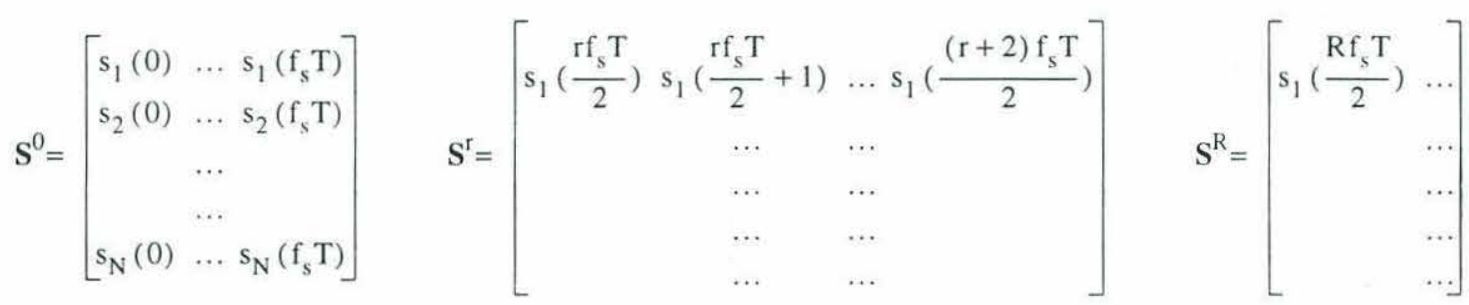

Outer Product Operation: The column vectors $\mathbf{S}^{\mathrm{r}}$ are used for the matrix outer product operation to form the sample covariance matrices. These matrices are sent to the angular power estimation algorithm.

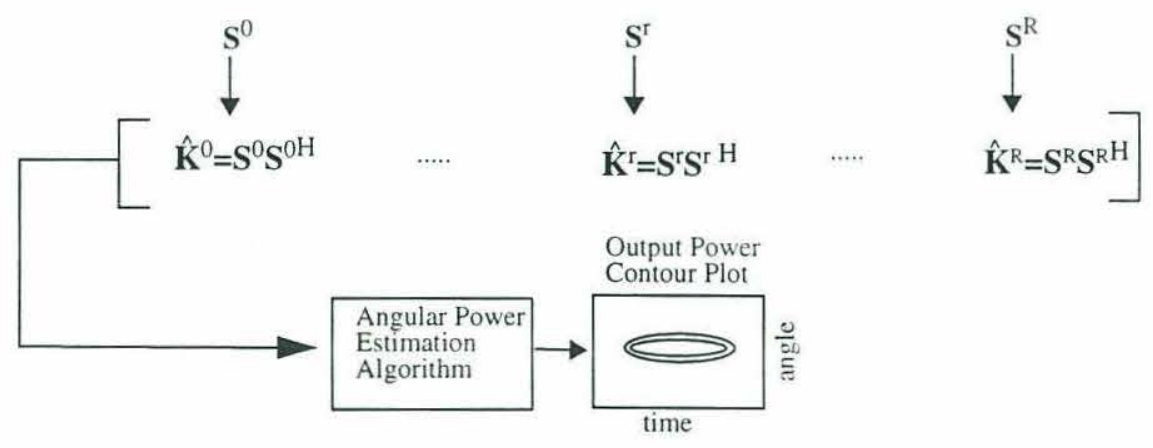


For example the time series from the second $(\mathrm{r}=1)$ time window and $\mathrm{nth}$ channel is given by equation 3.6. Note that the window length must be a integer multiple of $2 / \mathrm{f}_{\mathrm{s}}$ so the sample index is an integer.

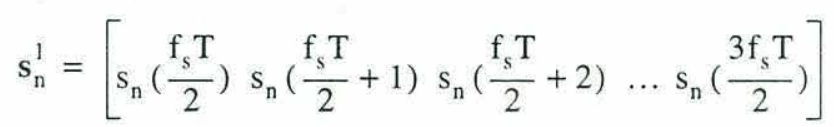

The $(\mathrm{j}, \mathrm{k})$ th element of the sample covariance matrix is formed by an inner product operation on the time series, thus the phase and magnitude is averaged over a time scale of $\mathrm{T}$ seconds.

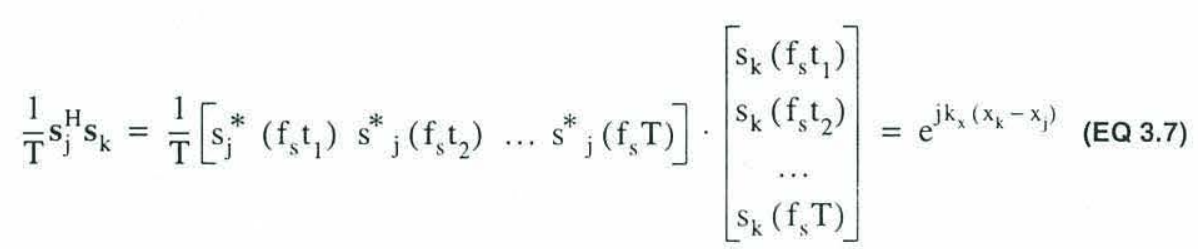

Here $s *$ is the complex conjugate of $\mathrm{s}$. The equality on the right side of this equation holds true for the plane wave signal model. Thus the elements of the sample covariance matrix contain the relative phase difference between the received channels. The length of this window is chosen to satisfy time bandwidth product considerations involved in determining the rank of the covariance matrix (see section 3.4.1.2).

When examining synthetic signals the power response of the beamformer is plotted vs. angle. For the data received on the array the angular power spectrum changes in time so a time series of sample covariance matrices is formed according to the windowing scheme shown in figure 3.5. The final output of the angular power processor is displayed in terms of a contour plot with power on the $\mathrm{z}$ axis.

\subsection{Adaptive processing}

Adaptive beamforming has two distinct advantages: the absence of sidelobes when it works at high SNR, and higher resolution vs. conventional methods. The principle disadvantages of adaptive processing are that it involves an inverse of the covariance matrix which is sensitive to both the rank of the matrix or any mismatch of the plane wave model. This inverse is accomplished through an eigenvalue decomposition which can give additional insight into the signal structure. Two types of adaptive beamforming methods are used. The Minimum Variance, Distortionless Filter (MVDF) was first proposed by Capon as the maximum likelihood method (MLM)[5]. Here it is examined in terms of an eigenvalue decomposition[3]. This approach leads to a signal and noise subspace decomposition method known as MUSIC (MUltiple SIgnal Classification, Schmidt (1986)[4].) 


\subsubsection{Minimum variance beamforming}

The basic principle of the minimum variance (MV) beamformer is to minimize the output variance of the beamformer subject to a constraint of unity gain in the direction of the steering vector. A beampattern using the AHRA for a minimum variance beamformer in response to a plane wave from $212^{\circ}$ is shown in figure 3.6 to illustrate this principle and the MV response is compared to the conventional response. In the context of the HIFT, where the goal is to determine an accurate direction of arrival or detect closely spaced multipath, the advantages of the adaptive beampattern are clearly visible.

\section{FIGURE 3.6}

MV beamforming compared to Conventional (uniform taper.)

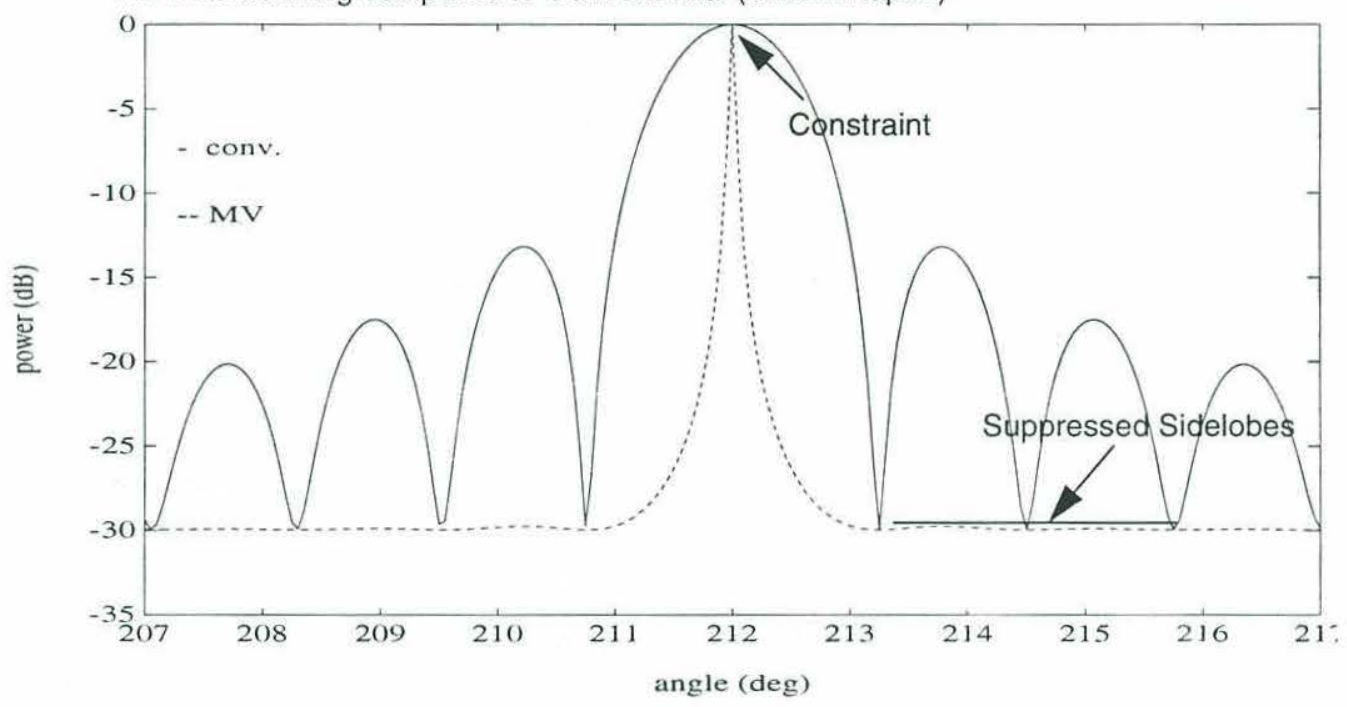

The typical method of deriving the MV beamformer is a constrained optimization problem which can be solved by variety of methods. This is not performed here since it is widely available in the literature. The result for power from the steering direction is stated as [5]:

$$
P(\theta)=\frac{1}{\mathbf{d}^{\mathrm{H}} \mathbf{K}^{-1} \mathbf{d}}
$$

$\mathbf{K}$ is the covariance matrix and $\mathbf{d}$ is the steering vector. The actual covariance matrix is not available, although an estimate of the covariance is formed in the sample covariance matrix as defined by the outer product $\hat{\mathbf{K}}=\mathrm{SS}^{\mathrm{H}}$. From the sample covariance matrix the power from direction $\theta$ is estimated.

$$
\hat{P}(\theta)=\frac{1}{\mathbf{d}^{\mathrm{H}} \hat{\mathbf{K}}^{-1} \mathbf{d}}
$$




\subsubsection{Sample covariance matrix vs. true covariance}

A key issue in adaptive processing is whether the sample covariance matrix adequately estimates the true covariance for the purpose of resolving multipath signals. The form of an element of the true spatial covariance at an instant in time for a narrow band process is given by:

$$
\mathbf{K}_{\mathrm{i}, \mathrm{j}}=\mathrm{E}\left[\left\{\mathrm{s}_{\mathrm{i}}^{*}\left(\mathrm{t}, \mathrm{x}_{\mathrm{i}}\right)\right\}\left\{\mathrm{s}_{\mathrm{j}}\left(\mathrm{t}, \mathrm{x}_{\mathrm{j}}\right)\right\}\right] .
$$

This quantity is estimated by:

$$
\hat{\mathbf{K}}_{\mathrm{i}, \mathrm{j}}=\frac{1}{\mathrm{~T}} \sum_{\mathrm{f}_{\mathrm{s}} \mathrm{t}=0}^{\mathrm{T}}\left[\left\{\mathrm{s}^{*}{ }_{\mathrm{i}}\left(\mathrm{f}_{\mathrm{s}} \mathrm{t}, \mathrm{x}_{\mathrm{j}}\right)\right\}\left\{\mathrm{s}_{\mathrm{j}}\left(\mathrm{f}_{\mathrm{s}} \mathrm{t}, \mathrm{x}_{\mathrm{j}}\right)\right\}\right] .
$$

The assumption inherent is using this estimate is that the expected value of the sample covariance matrix is equal to the true covariance.

$$
\mathbf{K}=\mathrm{E}(\hat{\mathbf{K}})=\lim _{\mathrm{T} \rightarrow \infty} \frac{1}{\mathrm{~T}}\left(\mathbf{S S}^{\mathrm{H}}\right)
$$

Since angular resolution is important in detecting multipath, small biasing effects on the magnitude of this estimate are not of great concern. Large biasing effects due to insufficient rank of the sample covariance matrix are very important. The ability to resolve two signals relies on allowing uncorrelated signals incident on the array to remain uncorrelated in the sample covariance matrix. The motivation for this becomes more evident when adaptive beampattern responses are examined for correlated and uncorrelated signals (see section 3.4.3.) The length of the time window used to form the sample covariance matrix and the bandwidth of the signal determine if this criteria can be met.

\subsubsection{Time bandwidth product considerations}

In the preliminary signal conditioning the received signals are filtered to have a bandwidth of $\pm 10 \mathrm{mHz}$. If a typical time window length of $\mathrm{T}=400 \mathrm{sec}$ is used, the uncorrelated frequency bins are approximately $1 / \mathrm{T}=2.5 \mathrm{mHz}$ apart. Thus the maximum number of uncorrelated signals in this covariance matrix is $20 \mathrm{mHz} / 2.5 \mathrm{mHz}=8$, which is the time bandwidth product.

This can also be interpreted in the time domain using the idea of independent snapshots of data. Since the signal has been filtered to $20 \mathrm{mHz}$ bandwidth the temporal decorrelation length is approximately $1 / 20 \mathrm{mHz}=50 \mathrm{~s}$. If the time window used to form the covariance matrix is $\mathrm{T}=400 \mathrm{~s}$. long the maximum number of independent snapshots of data is $400 / 50=8$. 


\subsubsection{Rank of the sample covariance matrix vs. the true covariance matrix}

1. Upper bound

For the case of the true covariance the upper bound of the rank is the number of array elements. The sample covariance matrix may contain less independent snapshots of data than the number of array elements. Thus the rank of the sample covariance matrix is equal to the number of independent data snapshots or the number of array elements, whichever is lesser. Although an upper bound on the rank of the sample covariance exists the following example shows how the rank may not equal its upper bound. The effective rank of the covariance matrix is determined by the structure of the signal. This example also demonstrates some of the differences between the true ensemble covariance the estimated sample covariance.

2.a Effective rank of the ensemble covariance matrix

If a model consisting of two plane waves $\left(\mathbf{S}_{0}, \mathbf{S}_{1}\right)$ from any directions including the same with no noise is considered, the ensemble covariance matrix takes the form:

$$
\begin{gathered}
\mathbf{K}=\mathrm{E}\left\{\frac{1}{\mathrm{~T}}\left(\mathbf{S}_{0}+\mathbf{S}_{1}\right)\left(\mathbf{S}_{0}+\mathbf{S}_{1}\right)^{\mathrm{H}}\right\} \\
\mathbf{K}=\mathrm{E}\left\{\frac{1}{\mathrm{~T}}\left(\mathbf{S}_{0} \mathbf{S}_{0}{ }^{\mathrm{H}}+\mathbf{S}_{1} \mathbf{S}_{1}^{\mathrm{H}}\right)\right\}+\mathrm{E}\left\{\frac{1}{\mathrm{~T}}\left(\mathbf{S}_{0} \mathbf{S}_{1}^{\mathrm{H}}+\mathbf{S}_{1} \mathbf{S}_{0}^{\mathrm{H}}\right)\right\}
\end{gathered}
$$

It is important to remember here that the signal vectors $\mathbf{S}$ are actually matrices as shown in section 3.3 since each element of the signal vector is a row vector of windowed time series data samples from one channel. This allows $\mathbf{K}$ in equation 3.11 to have rank greater than one. If the time series of that form the inner product operation of each element of the covariance matrix are perfectly uncorrelated then the expectation value of the second term involving the cross products is exactly zero. Then the covariance can be written as:

$$
\mathbf{K}=\mathrm{E}\left\{\frac{1}{\mathrm{~T}}\left(\mathbf{S}_{0} \mathbf{S}_{0}^{\mathrm{H}}+\mathbf{S}_{1} \mathbf{S}_{1}^{\mathrm{H}}\right)\right\}
$$

In the terms $\mathrm{S}_{0} \mathrm{~S}_{0}{ }^{\mathrm{H}}, \mathrm{S}_{1} \mathrm{~S}_{\mathrm{I}}{ }^{\mathrm{H}}$ the time dependance is cancelled by the complex conjugation so unless the signals are from the same direction $\left(\mathrm{S}_{0} \mathrm{~S}_{0}{ }^{\mathrm{H}}=\mathrm{S}_{1} \mathrm{~S}_{1}{ }^{\mathrm{H}}\right)$ then $\mathbf{K}$ is a rank two matrix. The directional uncorrelation angle is approximated by $\mathrm{k}_{\mathrm{x}}=2 \pi / \mathrm{L}$ where $\mathrm{L}$ is the length of the array. The location $\theta=\sin ^{-1}(\lambda / \mathrm{L})$ is the angle of the first null in the conventional uniform taper beampattern. For the case of two signals and no noise the effective rank is equal to the true rank of two in this example. The case of a signals with noise is considered in the next section. 
2.b Effective rank of the sample covariance matrix

For the same signal model the sample covariance matrix is:

$$
\begin{gathered}
\hat{\mathbf{K}}=\frac{1}{\mathrm{~T}}\left(\mathbf{S}_{0}+\mathbf{S}_{1}\right)\left(\mathbf{S}_{0}+\mathbf{S}_{1}\right)^{\mathrm{H}} \\
\hat{\mathbf{K}}=\frac{1}{\mathrm{~T}}\left(\mathbf{S}_{0} \mathbf{S}_{0}{ }^{\mathrm{H}}+\mathrm{S}_{1} \mathbf{S}_{1}^{\mathrm{H}}\right)+\frac{1}{\mathrm{~T}}\left(\mathbf{S}_{0} \mathbf{S}_{1}^{\mathrm{H}}+\mathrm{S}_{1} \mathbf{S}_{0}^{\mathrm{H}}\right)
\end{gathered}
$$

Under the assumption of ergodicity in the limit as the window length becomes infinite the sample covariance converges to the ensemble covariance.

$$
\mathbf{K}=\mathrm{E}(\hat{\mathbf{K}})=\lim _{\mathrm{T} \rightarrow \infty} \frac{1}{\mathrm{~T}}\left\{\left(\mathbf{S}_{0} \mathbf{S}_{0}{ }^{\mathrm{H}}+\mathbf{S}_{1} \mathbf{S}_{1}^{\mathrm{H}}\right)+\left(\mathbf{S}_{0} \mathbf{S}_{1}^{\mathrm{H}}+\mathbf{S}_{1} \mathbf{S}_{0}^{\mathrm{H}}\right)\right\}
$$

In this limiting process if $\mathbf{S}_{0}$ and $\mathbf{S}_{1}$ are uncorrelated the second term involving the cross products goes to zero as $1 / \mathrm{T}$. The first term remains finite. In the sample covariance matrix the window length is finite. In order for the cross terms to become small the window length should be much larger than the number of uncorrelated signals. If the window length is not long enough to allow the signals to decorrelate then the effective rank becomes less than the number of signals. The actual rank may still equal the number of uncorrelated signals if the window is long enough to allow the signals to partially decorrelate. In section 3.4.3 it is shown that the resolution of the adaptive beamformers depends on the degree of correlation between signals.

Thus if there is no noise the lower bound for the actual rank of the sample covariance matrix is the number of uncorrelated signals in a time window assuming there are an adequate number of independent snapshots. In reality there is always some noise in the signal from each array element. If the noise is uncorrelated from sensor to sensor the covariance matrix has rank equal to its upper bound. This kind of noise is spatially band-limited white noise and if an adequate number of snapshots are used it can be approximated as a matrix with only a constant term on the main diagonal since all the cross terms due to different uncorrelated channels are small.

$$
\hat{\mathbf{K}} \rightarrow \mathbf{K}+\sigma_{\text {noise }}^{2} \mathbf{I}
$$

In the synthetic signals generated for testing the adaptive array, band-limited white noise is added to avoid a singular matrix. Unless otherwise noted the band-limited white noise power level ( $\sigma_{\text {noise }}^{2}$ ) is set at $30 \mathrm{~dB}$ below the signal power in the synthetic data. In reality, the noise on the array is not necessarily band-limited white. This is one of the motivations for using the MUSIC beamforming method.

If the noise level is very small and the number of uncorrelated signals is less than the upper bound on the rank the sample covariance matrix may be poorly conditioned. This is discussed in greater detail in section 3.4.1.5 where an eigenvalue decomposition of the sample covariance matrix is considered. 
Assuming that the noise has enough independent elements so the sample covariance matrix has rank equal to the number of snapshots the order of the matrix should be no greater than the rank to avoid a singular inverse [6]. In this case there are 8 snapshots in each time window and the order is equal to the number of channels. In the conventional arrays 32 channels are used so this would clearly lead to a singular or nearly singular matrix inversion.

\subsubsection{Reduction of the order of the covariance matrix}

One solution to this problem would be to simply undersample the CHRA on every fourth element and end up with a 8 element array. The array gain vs. white noise associated with $\mathrm{N}=32$ elements drops by $10 \log 10\left(\frac{32}{8}\right)=6 \mathrm{~dB}$ with $\mathrm{N}=8$ elements. A better solution is to phase delay and sum every four adjacent channels in the correct direction to create 8 output channels. This preliminary beamforming operation, which requires knowing the general direction of the signal, is computed by the inner product $\mathrm{N}^{-1} \mathbf{d}^{\mathrm{H}} \mathrm{S}$. The steering vector $\mathbf{d}$ is a four element vector and the signal matrix $\mathbf{S}$ contains time series from four adjacent channels. The power is not calculated since this is a spatial filtering process. The output of this spatial filtering operation is a time series. This new array (AHRA) of signals has a inter-element spacing of $\Delta x=152.4 \mathrm{~m}$ so the angle between spatial aliasing lobes is $9.7^{\circ}$. Processing using this array configuration is limited to high resolution views of the angular peak of the signal. This method is also used in matched field processing and is known as subarray processing [7].

This summing of input channels increases the SNR of the input to the adaptive processor as seen in figure 3.7. The higher input SNR is offset by the lower array gain from the adaptive processor with fewer channels. The first case in this figure is superior since it results in a matrix inversion that is less sensitive to the noise.

FIGURE 3.7

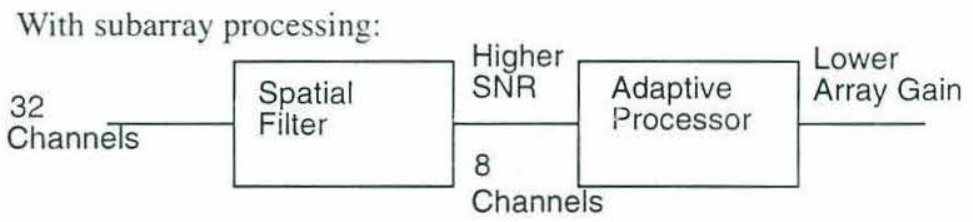

Without subarray processing:

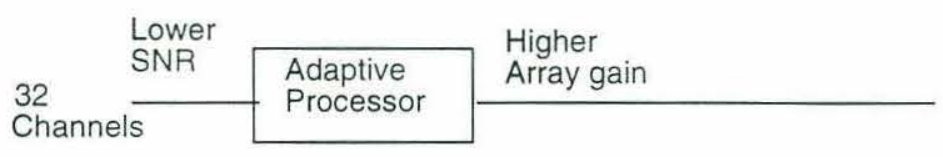

\subsubsection{Eigenvalue decomposition of the sample covariance matrix}

As mentioned previously, the inverse of the sample covariance matrix is achieved through an eigenvalue decomposition. The sample covariance matrix is always symmetric due to the fact that it is an outer product of a vector with its complex conjugate. This 
ensures that the eigenvectors are orthogonal (uncorrelated) to each other; they are chosen to be orthonormal and all the weighting is in the eigenvalue. The decomposition is written in the form:

$$
\hat{\mathbf{K}}=\Phi \Lambda \Phi^{\mathrm{H}}=\left[\begin{array}{llll}
\phi_{1} & \underline{\phi}_{\mathrm{i}} & \cdots & \phi_{\mathrm{N}}
\end{array}\right]\left[\begin{array}{cccc}
\lambda_{1} & 0 & 0 & 0 \\
0 & \lambda_{\mathrm{i}} & 0 & 0 \\
0 & 0 & \ldots & 0 \\
0 & 0 & 0 & \lambda_{N}
\end{array}\right]\left[\begin{array}{c}
\phi_{1}^{\mathrm{H}} \\
\phi_{1}^{\mathrm{H}} \\
\underline{\mathrm{i}}_{\mathrm{i}} \\
\cdots \\
\phi_{\mathrm{N}}^{\mathrm{H}}
\end{array}\right]
$$

\section{Eigenvectors Eigenvalues Eigenvectors ${ }^{\mathrm{H}}$}

To calculate the inverse, the eigenvalues $\lambda_{\mathrm{i}}$ are replaced with $1 / \lambda_{\mathrm{i}}$ in the eigenvalue diagonal matrix. Taking advantage of this matrix operation an estimate of the power from the steering direction is written as:

$$
\hat{\mathbf{P}}(\theta)=\frac{1}{\mathbf{d}^{\mathrm{H}} \hat{\mathbf{K}}^{-1} \mathbf{d}}=\left[\sum_{i=1}^{N} \frac{\left|\mathbf{d}^{\mathrm{H}} \cdot \phi_{i}\right|^{2}}{\lambda_{i}}\right]^{-1}
$$

where $\frac{\left|\mathrm{d}^{\mathrm{H}} \cdot \phi_{\mathrm{i}}\right|^{2}}{\lambda_{\mathrm{i}}}$ is the projection of the steering vector on the eigenvector weighted by $1 / \lambda_{\mathrm{i}}$. The first term of this sum, when evaluated in response to a synthetic plane wave over the range of steering directions, takes the form of a weighted sinc function as in conventional beamforming (figure 3.8). The nulls in the terms with small eigenvalues determine where the peaks in the output occur as a function of angle $(\theta)$. The power of each uncorrelated signal appears as the weighting in the eigenvalue. Since the eigenvalue is in the denominator the projections with the less powerful eigenvalues dominate the summation. The reason a powerful signal emerges as the peak in the beampattern is the signal causes a null in all the other weighted projections due to the orthogonality. This is illustrated graphically in figure 3.8 and 3.9 by examining the weighted projections of the first few eigenvectors and then summing the terms graphically. The simulated data used for this figure is a plane wave from $212^{\circ}$ with a bandlimited white noise floor of $-30 \mathrm{~dB}$ with respect to the signal. This is the same as that used in figure 3.6.

In the graphic interpretation of the MV processor displayed in figure 3.8 and 3.9 the number of independent data snapshots is very large compared to the number of channels $(\mathrm{N}=8)$. Thus the estimate of the covariance matrix is a very accurate representation of the true covariance. In appendix A of this chapter a graphical interpretation is performed for the three cases of: i) number of snapshots $>>$ number of channels, ii) number of snapshots $=$ number of channels, iii) number of snapshots $<$ number of channels. This appendix illustrates the issues involved in adequately estimating the covariance with the sample covariance matrix. 

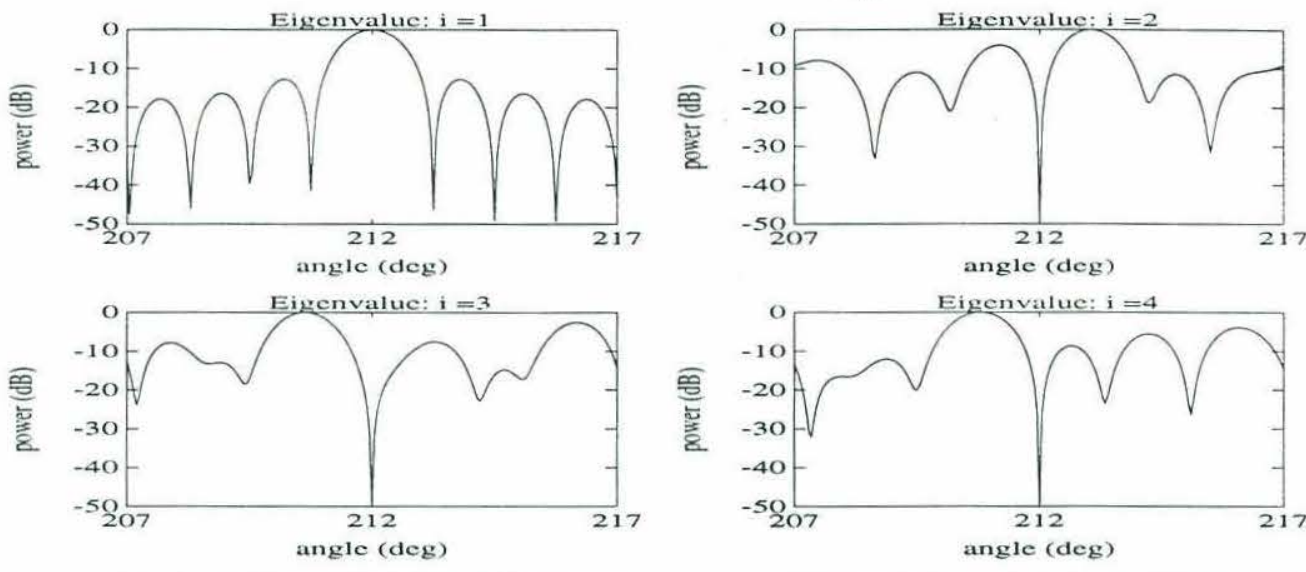

FIGURE 3.9

1/ Sum of Weighted Projections: $20 \log _{10}\left(\left[\sum_{\mathrm{i}=1}^{\mathrm{k}} \frac{\left|\mathbf{D}^{\mathrm{H}} \cdot \phi_{\mathrm{i}}\right|^{2}}{\lambda_{\mathrm{i}}}\right]^{-1}\right)$
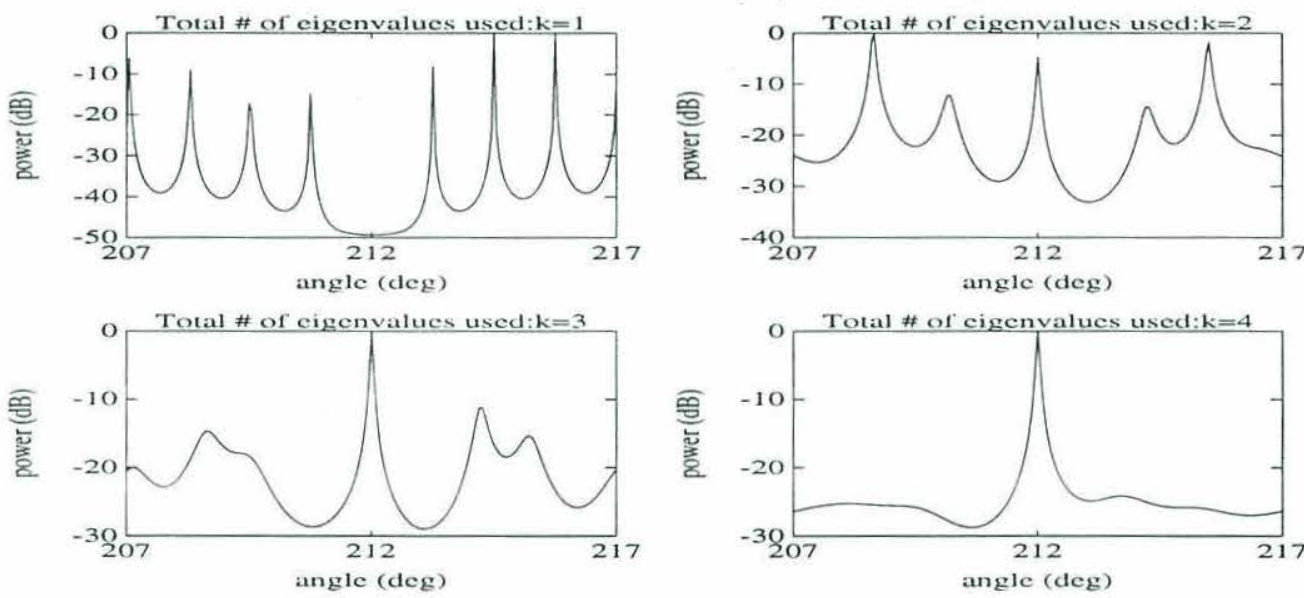

In figure 3.9, as more weighted projections are summed the peak at the signal location emerges, and due to the orthogonality of the remaining eigenvectors the response becomes increasingly flat outside the signal direction. By the fourth plot in figure 3.9 the response closely resembles the total response as seen in figure 3.6. This flatness relies on the orthogonality and equal weighting of the projections in the noise. The orthogonality is guaranteed by the eigenvalue decomposition since the sample covariance matrix is conjugate symmetric. The eigenvectors are only orthogonal with respect to equal weights. If the weights (eigenvalues) are not equal in the noise, the secondary nulls in the weighted projections with the smallest weights become peaks in the output. This may accurately represent peaks in the noise field, but can also lead to confusing results especially if eigenvalues are very small due to poor estimation of the sample covariance. If the noise eigenvalues are unequal, but very small, an effective method of equalizing them is to add a small amount of power to each one. This technique, known as diagonal loading, is accomplished by adding an identity matrix (scaled to some small fraction of the signal power) to the covariance matrix. If the noise eigenvalues are 
unequal, but close in power relative to the signal then this technique requires adding substantial amounts of noise power to the covariance matrix, which could make the signal difficult to detect. In this case a subspace decomposition technique results in improved performance as discussed in section 3.4.2.

\subsubsection{Eigenvalue spectrum}

In figure 3.10 there are four typical spectrums of eigenvalue magnitudes. The first (figure $3.10 \mathrm{a}$ ) is from the synthetic data with one plane wave incident on the array with spatially band-limited white noise. The signal subspace containing the plane wave at a power level of $0 \mathrm{~dB}$ is clearly distinguishable from the flat noise subspace at a power level of $-30 \mathrm{~dB}$. The second (figure $3.10 \mathrm{~b}$ ) contains two uncorrelated plane waves from directions $1.3^{\circ}$ apart, one $6 \mathrm{~dB}$ less powerful than the other. This is the minimum angular distance between the signals required to decorrelate them as indicated by the first null of the conventional response of this array (at approximately $\theta=$ 1/L). These signals are also clearly distinguishable from the noise subspace. The third (figure $3.10 \mathrm{c}$ ) shows two perfectly correlated plane waves from directions separated by $2.2^{\circ}$. These plane waves are not distinguishable from each other in the eigenvalue spectrum because they are correlated, but are distinguishable from the noise subspace. The beampattern for this case is compared to the second case in section 3.4.3. The fourth (figure $3.10 \mathrm{~d}$ ) shows a synthetically generated eigenvalue spectrum typical of the actual data received on the array. Note that the noise is not spatially white. The eigenvalues are scaled to exponentially decay vs. index with a $25 \mathrm{~dB}$ range. On the decibel plot the eigenvalues appear to decay linearly. In this case it is difficult to determine where the signal subspace ends and the noise subspace begins. The effects of the spatially colored noise are discussed in the next section. These examples are shown to demonstrate the power and limitations of the eigenvalue spectrum in analyzing the actual data.

FIGURE 3.10
Eigenvalue Spectrums

a: One Signal

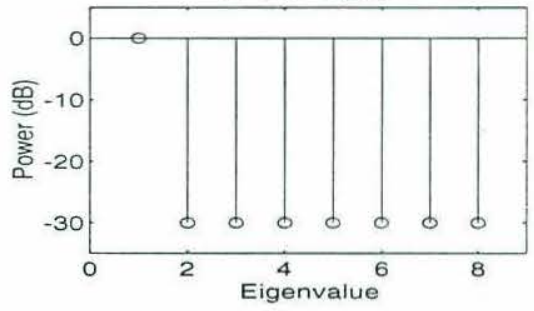

c: Two correlated signals

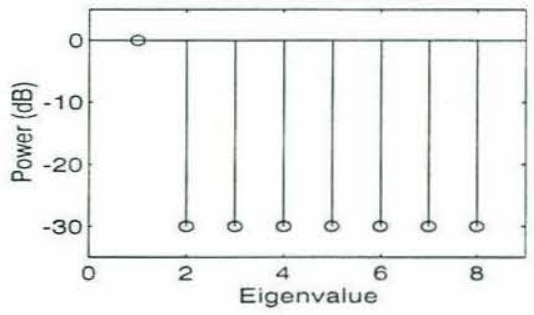

b: Two uncorrelated signals, $6 \mathrm{~dB}$ relative power

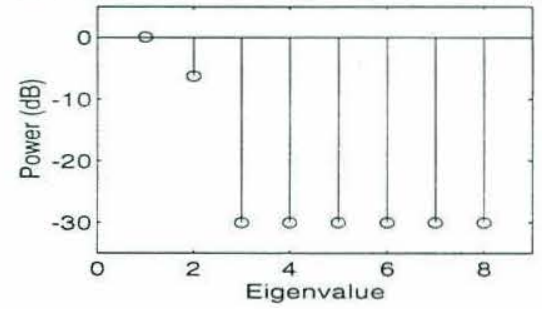

d: Synthetic decay spectrum

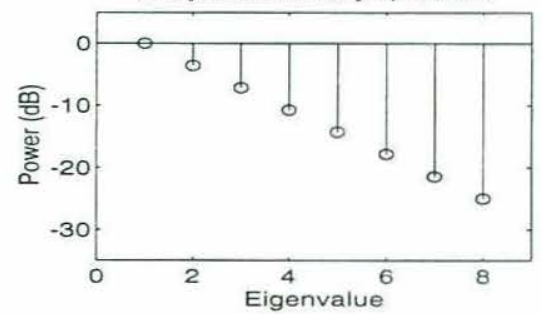




\subsubsection{MUSIC Beamforming}

The eigenvalue spectrum of figure $3.10 \mathrm{~d}$ is used to color the noise field and the same weighted projections as in figure 3.8 are used to form a new sample covariance matrix. The results of MV processing on this sample covariance matrix are shown in figure 3.11. The MV processor output is adversely affected by the colored noise field. To isolate the signal from the noise in the beamformed output, the eigenvalue spectrum can be artificially whitened in the noise subspace. This is performed by simply setting the eigenvalues in the noise subspace to unity. The signal eigenvalues are set to zero since only the nulls in the artificially whitened noise create the peaks in the output. Thus an estimate of the power from the MUSIC processor is:

$$
\hat{\mathrm{P}}(\theta)=\left[\sum_{\mathrm{i}=\mathrm{k}+1}^{\mathrm{N}}\left|\mathbf{d}^{\mathrm{H}} \cdot \phi_{\mathrm{i}}\right|^{2}\right]^{-1} .
$$

The $\mathrm{i}=1$ to $\mathrm{k}$ terms are the eigenvalues and vectors in the signal subspace, and $\mathrm{k}+1$ to $\mathrm{N}$ terms of the sum are in the noise subspace. This gives the theoretic possibility of infinite white noise gain since the nulls can approach zero in the noise subspace. The difficult part of this is to decide where the signal subspace ends and the noise subspace begins. If there is a clear step as in the synthetically generated spectrums of figure 3.10 it is easy. In cases where there is no clear step one can decide how many signals to look for and use that number of eigenvalues in the signal subspace. The MUSIC result is compared to the $\mathrm{MV}$ result in a colored noise field in figure 3.11. There are many other ways to treat this problem of small unequally weighted eigenvalues in a colored noise field. This method is chosen because of its ability to isolate signal from noise.

FIGURE 3.11

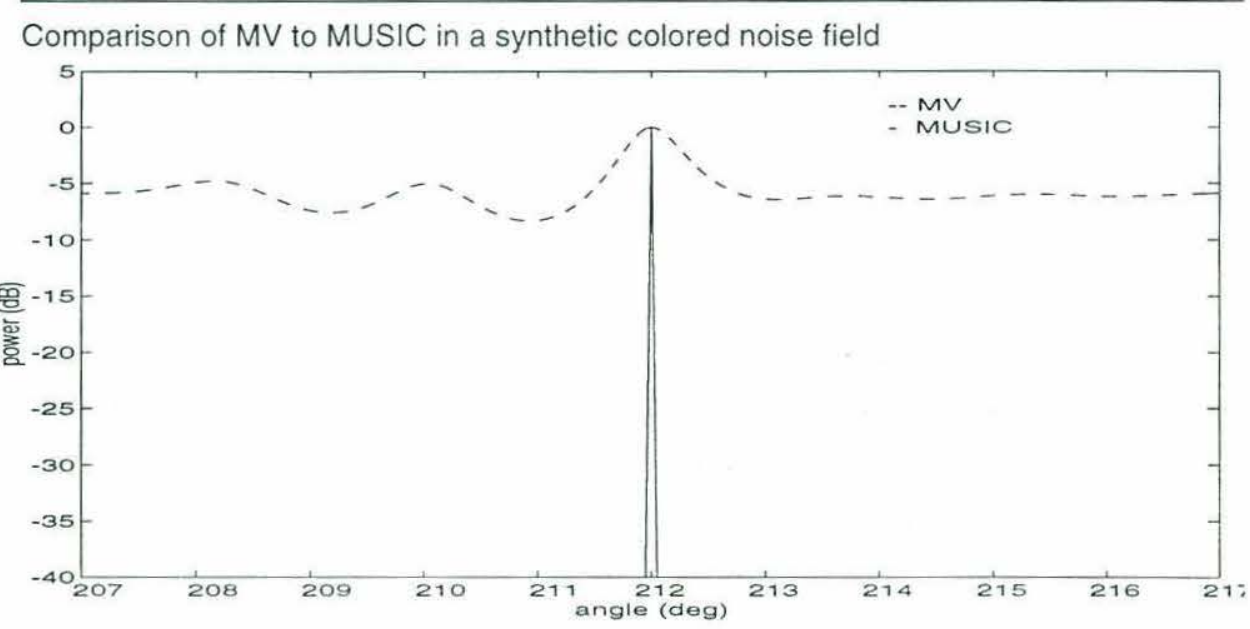

\subsubsection{Performance Evaluation of Adaptive Beamformers}

The angular resolution of the adaptive processors for one plane wave is limited by the noise field as seen in figure 3.12. The angular width of the signal itself, or imperfect knowledge of the array geometry can also limit the resolution of the adaptive beam- 
former. There are no preset resolution limits as in conventional beamforming. The performance of the adaptive beamformers for resolving two plane waves is examined by the same technique that is used for evaluating conventional beamforming. The performance for correlated and uncorrelated plane waves with the MV and MUSIC processors is compared in figure 3.12 .

\section{FIGURE 3.12}

\section{Multiple Plane Wave Resolution: Adaptive Processors}

Uncorrelated signals: MUSIC Eigenvalues used:2

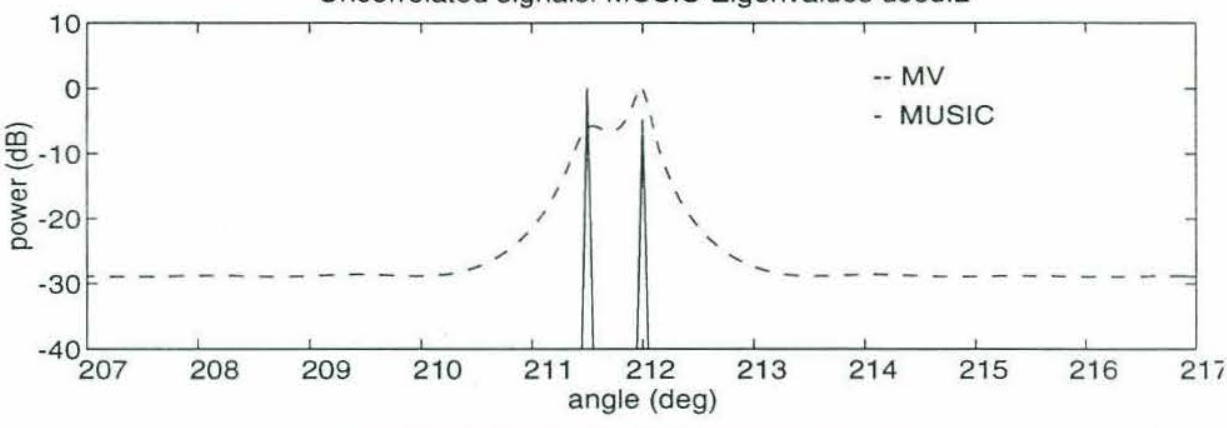

Correlated signals: MUSIC Eigenvalues used:2

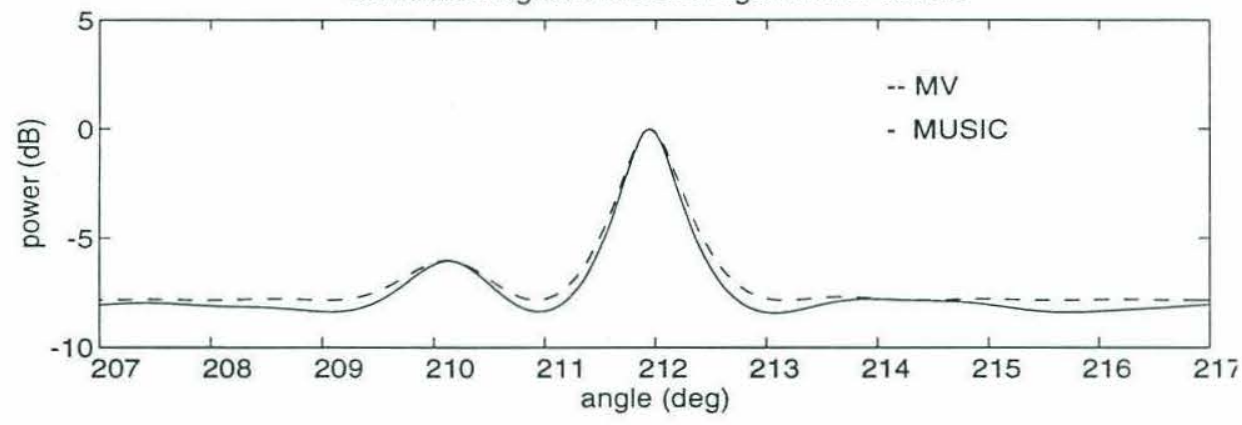

The synthetic uncorrelated signals in figure 3.12 are $0.5^{\circ}$ apart. The MV processor is barely able to resolve between the two signals while the MUSIC processor is able to clearly resolve the two signals. Unfortunately in real data processing a plane wave may not be able to be resolved so precisely due to imperfections of the wave front itself or sampling problems. It is seen from this figure however that the MUSIC processor can reveal the fine structure of the peak in the directional power spectrum. One drawback of a method such as MUSIC is that the amplitude of the peak does not accurately represent the power in the signal. In figure 3.12 the plane waves were synthetically designed to have a relative power difference of $6 \mathrm{~dB}$. This is accurately reflected in the MV processors output, but the MUSIC processor shows the signal at $212^{\circ}$ having more power than the signal at $211.5^{\circ}$ ! The reason for this is shown in appendix A of this chapter.

The adaptive processors have poor performance in resolving two correlated plane waves. There is little difference between the MV and MUSIC processor since both signals directions are in one eigenvector and the eigenvalue decomposition can not separate the signals. The minimum angular distance required to barely resolve the two signals increases to $2^{\circ}$. The noise floor appears to be $-8 \mathrm{~dB}$ while it is really $-30 \mathrm{~dB}$ as 
seen in the uncorrelated comparison. The performance is actually worse than the conventional beamformer with a uniform taper, which can resolve signals $1.6^{\circ}$ apart, and accurately capture the noise field within the limits of the sidelobe structure. Thus the possible correlation of multipath arrivals could make detection a difficult problem.

FIGURE 3.13
Horizontal Refraction model

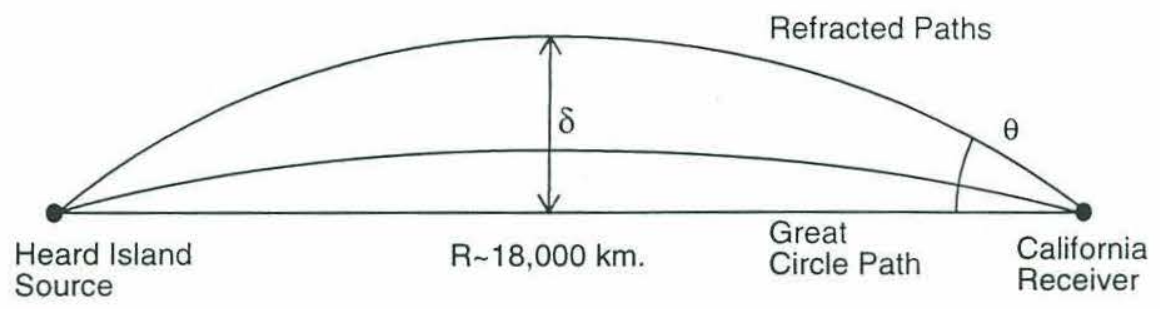

As shown in figure 3.13 a small difference in horizontal angle may cause the multipaths to sample different oceanographic regions with different time and space dependent processes, which cause the multipath to decorrelate. As the signals become closer together in arrival angle they are more likely to correlate and this causes the adaptive beamformers' signal resolution abilities to degrade. Thus the correlated signal beampatterns of figure 3.12 show the worst case resolution limits, with the signals still correlated even with $2^{\circ}$ arrival angle separation. For ideal multipath resolution the signals would tend to decrease their correlations as the arrival angle separation decreases, but this is not the case as the geometry of figure 3.13 indicates. Assuming the separation angle that correlates the multipath due to traveling similar paths is less than $2^{\circ}$, then the separation angle where correlation occurs is the resolution limit of the adaptive beamformers. Unfortunately this separation angle is not known and predicting it requires modeling acoustic propagation through oceanographic mesoscale random processes.

\subsection{Array geometry sensitivity}

\subsubsection{Deterministic model}

One of the principle limitations of the beamforming operation is its sensitivity to array geometry. The inter-element spacing that has been used for the generation of synthetic data is measured on the actual array and does not change during the course of the tow. The array may not be aligned along a straight line as has been assumed for the synthetic data. It is difficult to have exact knowledge of the array curvature at all times. To determine the beamformers' sensitivity to array curvature, synthetic data with one incident plane wave at $212^{\circ}$ is generated using a curved version of the AHRA array, but is beamformed assuming the array is straight. To account for the off-axis displacement of the 
array the form of the signal received at the ith element of the array (equation 3.1) is changed slightly to:

$$
S_{i}=e^{j k\left(x_{i} \cdot \sin \theta+y_{i} \cdot \cos \theta\right)}
$$

The curvature used for generating the synthetic data is a half period sine wave along the length of the array. This assumes a smooth curvature with no sign change in the second derivative with respect to the $x$-axis which may not be the case. The amplitude of the sinusoid is varied in figure 3.14 to determine the beamformer's sensitivity to array curvature.

\section{FIGURE 3.14}

\section{Array Curvature Sensitivity}

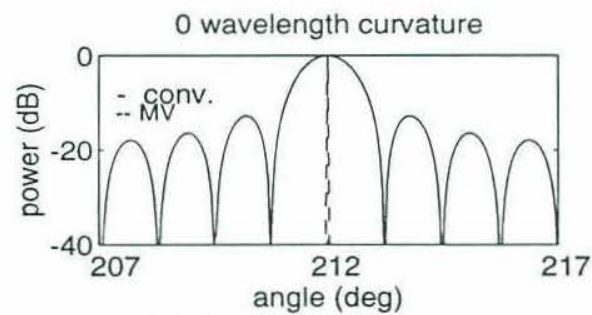

$1 / 16$ wavelength curvature

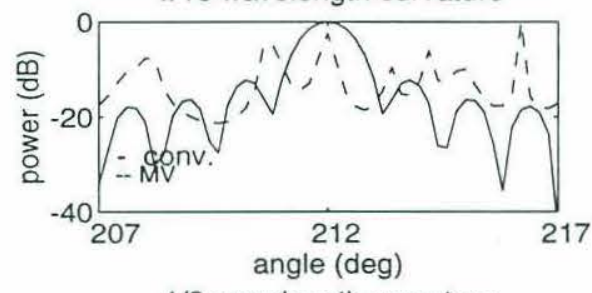

$1 / 8$ wavelength curvature

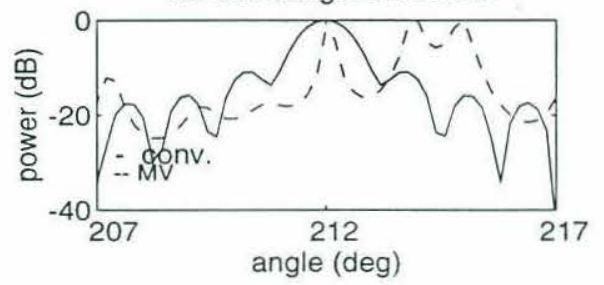

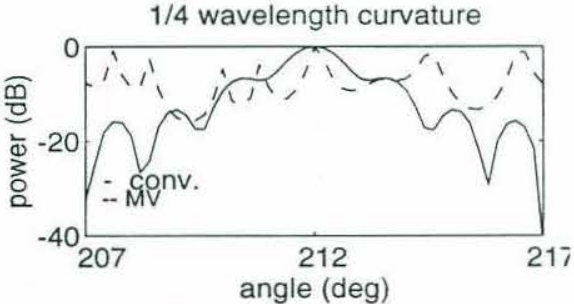

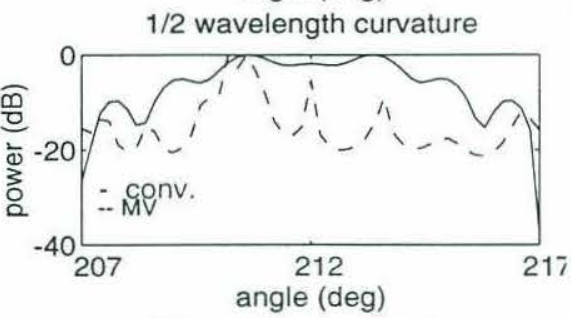

$3 / 4$ wavelength curvature

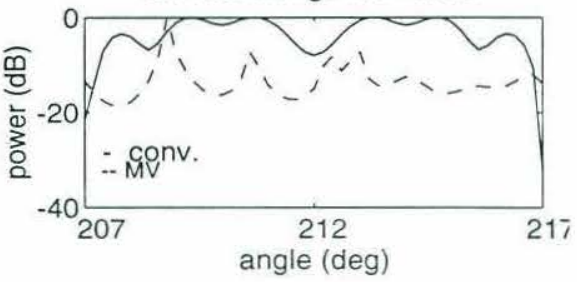

As seen in the plots of figure 3.14 the MV processor is very sensitive to mismatch in the array geometry while the conventional method is much more robust. This type of analysis is also performed with the MUSIC processor and the results, while not deteriorating as badly at $1 / 16$ and $1 / 8$ of a wavelength curvature, are unacceptable for curvatures $1 / 4$ wavelength or greater. The eigenvalue spectrum is not adversely affected by array curvature as is seen by figure 3.15 . For figure 3.15 the two synthetic plane waves are generated using the curved array. The plane waves are $6 \mathrm{~dB}$ apart in power and $1.6^{\circ}$ apart in angle. This is sufficient to decorrelate the signals on a straight array. It is seen in the figure that the curvature does not cause the plane waves to correlate, as the first two eigenvalues remain $6 \mathrm{~dB}$ apart and no energy is transferred to higher eigenvalues. The eigenvalue decomposition does not require any knowledge of the plane wave model. The sampling issues that are required to proceed from equation 3.10 to 3.11 are also met with a curved array. 


\section{FIGURE 3.15}

\section{Eigenvalue Spectrum Sensitivity to Array Geometry}

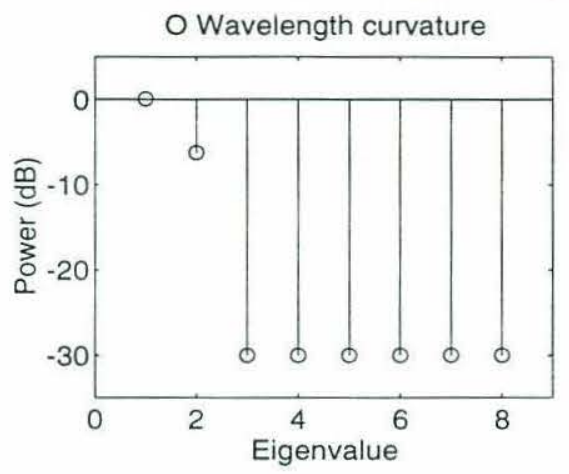

$1 / 4$ Wavelength curvature

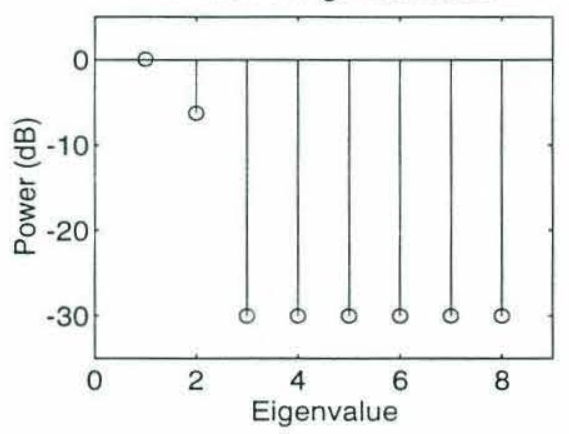

$1 / 2$ Wavelength curvature

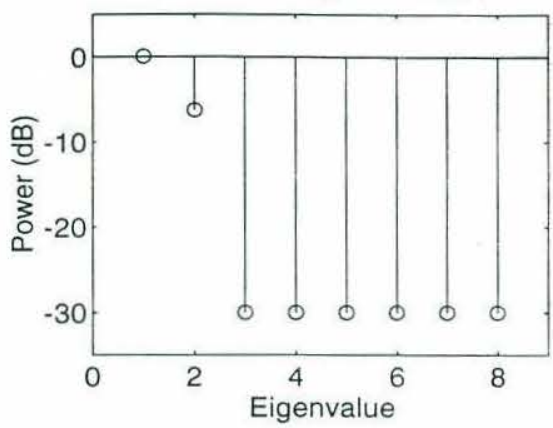

$3 / 4$ Wavelength curvature

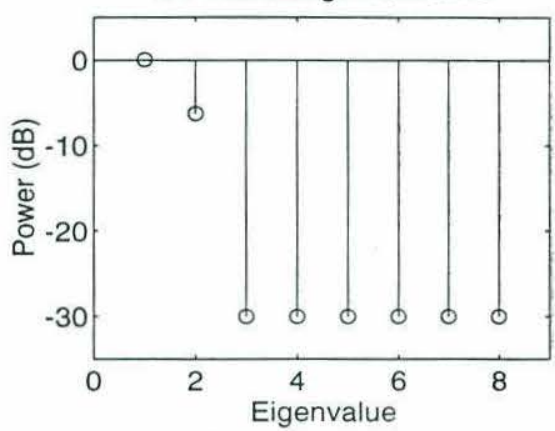

\subsubsection{Stochastic model}

An alternative method of analyzing the sensitivity to geometry errors is to add a random position error to each array element and determine the mean and the variance of the beamformer output. The errors added to the element positions are assumed to be statistically independent random variables. Independence is not a entirely physical assumption since the array elements are constrained to be on the array. An accurate stochastic model would incorporate statistics of array curvature. This could be used to determine the correlations between array element position errors. This approach is beyond the scope of this thesis since the data available on the array curvature is determined by three heading sensors. Only statistics on the first two modes of curvature can be obtained while higher order modes may exist.

The previous plane wave signal vector model of

$$
s_{i}=e^{j k_{x} x_{1}}
$$

is modified assuming a position error model of

$$
\mathrm{x}_{\mathrm{i}} \rightarrow \overline{\mathrm{x}}_{\mathrm{i}}+\Delta \mathrm{y}_{\mathrm{i}} .
$$


This model assumes the error is off the axis of the line array. This is a realistic assumption for a horizontal line array where the elements are a fixed distance apart. but array may curvature may exist. If the analysis is restricted to signal arrival angles close to broadside then $k_{y} \approx k$. These assumptions allow the deterministic model for array curvature discussed in the previous section to be compared to the stochastic model in a qualitative sense.

The plane wave model now becomes

$$
S_{i}=e^{j k_{x} \bar{x}_{i}} \cdot e^{j k \Delta y_{i}} .
$$

Defining the phase error as $k \Delta y_{i}=\Delta \phi_{i}$ and assuming the $\Delta \phi_{i}$ are statistically independent zero-mean Gaussian random variables with variance $\sigma_{\phi}^{2}$, the expectation of the beamformer output is taken as

$$
E\left(\frac{d^{H} s}{N}\right)=\frac{1}{N} E\left(\sum_{i=1}^{N} d_{i} s_{i}\right)=\frac{1}{N} \sum_{i} d_{i} \bar{s}_{i} E\left(e^{j \Delta \phi_{i}}\right) .
$$

Taking advantage of the Fourier Transform of a Gaussian function the expectation within the summation is evaluated as

$$
E\left(e^{j \Delta \phi_{1}}\right)=\int_{-\infty}^{\infty} e^{j \Delta \phi_{i}} \cdot \frac{e^{-\left(\Delta \phi_{1}^{2} / 2 \sigma_{\phi}^{2}\right)}}{\sqrt{2 \pi} \sigma_{\phi}^{2}} d \Delta \phi_{i}=e^{-\sigma_{\varphi}^{2} / 2} .
$$

Thus the mean of the beamformer output is reduced by an exponential factor and now becomes:

$$
\frac{\mathbf{d}^{\mathrm{H}} \overline{\mathbf{s}}}{\mathrm{N}} \mathrm{e}^{-\sigma_{\varphi}^{2} / 2}
$$

The variance of the beamformer output is determined as

$$
\begin{gathered}
\sigma_{\frac{d^{H} s}{N}}^{2}=E\left\langle\left(\sum_{j} \frac{d_{j} s_{j}}{N}\right)\left(\sum_{i} \frac{d_{i} s_{i}}{N}\right)^{*}\right\rangle \\
=\sum_{i, j} \frac{E\left\langle\left(d_{j} s_{j}\right) \cdot\left(d_{i} s_{i}\right)^{*}\right\rangle}{N^{2}} \\
=\sum_{i}\left|\frac{d_{i}}{N}\right|^{2} \sigma_{s_{i}}^{2}
\end{gathered}
$$

where $\left(d_{i} s_{i}\right)^{*}$ is the complex conjugate of $d_{i} s_{i}$. 


$$
\begin{gathered}
\sigma_{\mathrm{s}_{1}}^{2}=E\left(\left|\mathrm{e}^{\mathrm{j} \Delta \phi_{1}}\right|^{2}\right)-E\left(\mathrm{e}^{\mathrm{j} \Delta \phi_{1}}\right)^{2} \\
=1-\mathrm{e}^{-\sigma_{\bullet}^{2}}
\end{gathered}
$$

Thus as the variance of the phase error increases, so does the variance of the beamformer output.

$$
\sigma_{\frac{d^{H} s}{N}}^{2}=\left(1-e^{-\sigma_{\varphi}^{2}}\right) \sum_{i}\left|\frac{d_{i}}{N}\right|^{2}
$$

In the conventional beamformer the $\mathrm{d}_{\mathrm{i}}$ are phase terms with unity magnitude. As the phase error variance becomes greater than two the output variance effectively becomes a constant $(1 / \mathrm{N})$ with respect to $\sigma_{\phi}$.

Although a direct comparison of the stochastic model to the deterministic is impossible due to the intrinsic differences between these methods a qualitative comparison can be made. To compare these results to the results of the deterministic approach the output variance is plotted vs. the amplitude of the sinusoidal array model curvature in figure 3.16. The scale of the phase variance is related to the amplitude of curvature by first determining the rms value of the curved model as

$$
\Delta \mathrm{y}_{\mathrm{rms}}=\frac{1}{\pi} \int_{0}^{\pi}\left(\mathrm{A} \sin \mathrm{x}-\frac{1}{\pi} \int_{0}^{\pi} \mathrm{A} \sin \mathrm{xdx}\right)^{2} \mathrm{dx}=0.0947 \mathrm{~A}^{2} .
$$

Thus $0.0947 \mathrm{~A}^{2} \approx \sigma_{\Delta y}^{2}$ where $\mathrm{A}$ is the amplitude of the curvature in wavelengths.

A factor of $2 \pi$ also arises due to

$$
\begin{gathered}
\mathrm{k} \Delta \mathrm{y}=\Delta \phi \\
\sigma_{\Delta y}^{2}=\frac{1}{(2 \pi)^{2}} \sigma_{\Delta \phi}^{2}
\end{gathered}
$$

where $\Delta \mathrm{y}$ is measured in wavelengths. The output variance is normalized by $\mathrm{N}$ in figure 3.16 .

\section{FIGURE 3.16}

Output error variance vs. Array curvature amplitude.

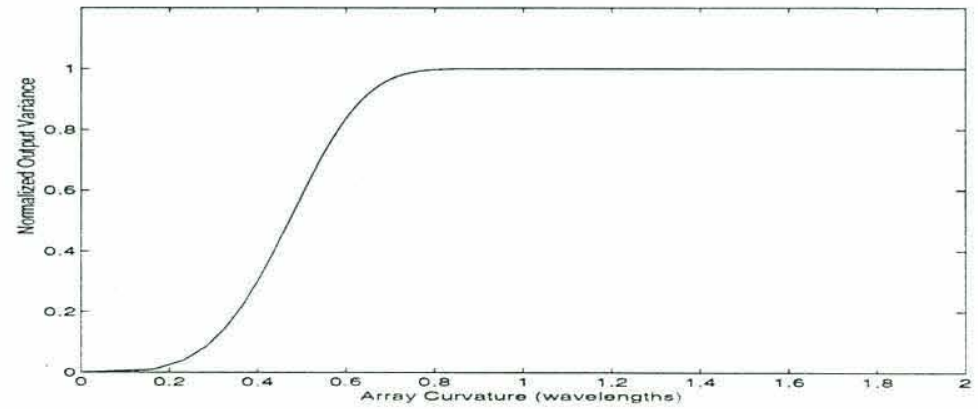


From figure 3.14 of the deterministic model, it is seen that the performance of the conventional beamformer deteriorates at curvature amplitudes greater than $1 / 4$ of a wavelength. In figure 3.16 this is where the variance increases sharply. The effects of the reduction of the mean of the beamformer output are not noticeable in figure 3.14 because the plots are normalized to have a maximum power of $0 \mathrm{~dB}$.

\subsubsection{Extension to adaptive processing}

The results of the previous section are extended to the adaptive processor by using a slightly different interpretation of the adaptive processor than is used previously in this thesis. Previously the only the power from the adaptive processor is considered. The adaptive processor can be interpreted as the inner product of a weight vector $\mathbf{w}$ with a signal vector $\mathbf{s}$ as discussed in conventional beamforming. For adaptive processing, the conventional beamformer weight vector of $\mathbf{w}=\mathbf{d} / \mathrm{N}$ is changed to

$$
\mathbf{w}=\frac{\hat{\mathbf{K}}^{-1} \mathbf{d}}{\mathbf{d}^{\mathrm{H}} \hat{\mathbf{K}}^{-1} \mathbf{d}}
$$

where $\mathbf{d}$ is the steering vector as used in conventional beamforming. Substituting this weight vector into equation 3.15 , for the mean of the beamformer output, it is evident that the position error reduces the mean of the output.

$$
\mathrm{E}\left(\mathbf{w}^{\mathrm{H}} \mathbf{s}\right)=\mathbf{w}^{\mathrm{H}} \overline{\mathbf{s}} \mathrm{e}^{-\sigma_{\phi}^{2} / 2}
$$

Using equation 3.16 for the variance of the beamformer output the variance of the adaptive processor becomes

$$
\sigma_{\mathbf{w}^{H} \mathrm{~s}}^{2}=\left(1-\mathrm{e}^{-\sigma_{\varphi}^{2}}\right) \mathbf{w}^{\mathrm{H}} \mathbf{w}
$$

Since the weight vector is dependent on the input sample covariance matrix the output variance cannot be determined without knowledge of the sample covariance matrix. Since the variance of the output of the adaptive processor is proportional to the length of the weight vector a large weight vector causes a large variance. The denominator of equation 3.17 for the weight vector is the same as the denominator of the power expression (equation 3.9) for the adaptive processor. Thus the power output is large when the denominator is small and the weight vector can become large. Since the adaptive processor is constrained to have unit gain in the steering direction the largest weight vectors results when the processor is steered near, but not at a plane wave.

The large weight vector of the adaptive processor causes very high sensitivity to array element position error. This is evident both from the stochastic model equation 3.19 and from the deterministic model, which in figure 3.14 shows poor performance with small amounts of array curvature magnitude. 
[1] D.W. Craig and L. Mckee, "High performance shipboard acoustics data acquisition, imaging," Sea Technology 31, No. 1,p. 51-53, January (1990).

[2] F. J. Harris (1977), "On the Use of Windows for Harmonic Analysis with the Discrete Fourier Transform," proc. of the IEEE, Vol. 66, No. 1, January 1978

[3] W. S. Burdic, "Underwater Acoustic System Analysis (2nd Edition)," PrenticeHall, Inc. 1991.

[4] Schmidt, R. O., "Multiple Emitter Location and Signal Parameter Estimation," IEEE Trans., AP34, No. 3, p. 276-280 (Mar. 1986).

[5] Capon, J., High resolution frequency wavenumber spectrum analysis," Proc. of the IEEE, 57(8) p. 1408-1418 (1969).

[6] Strang, G., "Introduction to Applied Mathematics," Wellesley-Cambridge Press, 1986.

[7] Cox, H. Zeskind, R.M. and Myers, M., "A subarray approach to matched-field processing," J. of the Acoustical Soc. of Amer, 87(1), p 168-178 (1990) 


\section{CHAPTER 3: Appendix A: Adaptive Processor Graphic Interpretation}

In these examples the projections of the eigenvectors on the steering vectors and the inverse sum of weighted projections are plotted for all 8 eigenvalues. The magnitudes of the eigenvalues are also plotted for each case.

The signal model for all cases is two plane waves from $212^{\circ}$ and $214^{\circ}$ separated by $6 \mathrm{~dB}$ in power. The noise model is slightly different than used in chapter 3 . In chapter 3 synthetic examples band limited white noise is added by adding a scaled identity matrix to the sample covariance matrix. This assumes the noise is truly uncorrelated from sensor to sensor. Here the concern is whether the number of snapshots involved in forming the sample covariance matrix accurately captures the true noise field, thus noise is added to the individual channels. The noise on each channel is a sequence of randomly generated numbers with zero mean, and variance $-30 \mathrm{~dB}$ below the maximum signal power. Since no spectral filtering is used here the number of snapshots is equal to the number of samples in each time window.

Three cases are considered:

Case 1:Number of Snapshots $\gg$ Number of Channels

Case 2:Number of Snapshots $=$ Number of Channels

Case 3:Number of Snapshots $<$ Number of Channels

For the third case diagonal loading and MUSIC are examined to overcome the poor condition of the estimated sample covariance matrix.

In the context of the horizontal beamforming results presented in chapter 4 the time window used to from the sample covariance matrix and the number of array elements are chosen so the number of snapshots equals the number of elements. This appendix demonstrates that this forms an adequate representation of the true covariance for the purpose of resolving two plane wave signals. This appendix also demonstrates how the MUSIC method performs the summation in the denominator of the adaptive power expression and how it achieves high resolution when the number of eigenvalues is chosen to correctly match the number of incoming signals. 
Case 1: $\left(\right.$ Number of Snapshots $\left.\left(N_{S}\right)=5000\right) \gg($ Number of Channels $(N)=8)$

FIGURE A3.17
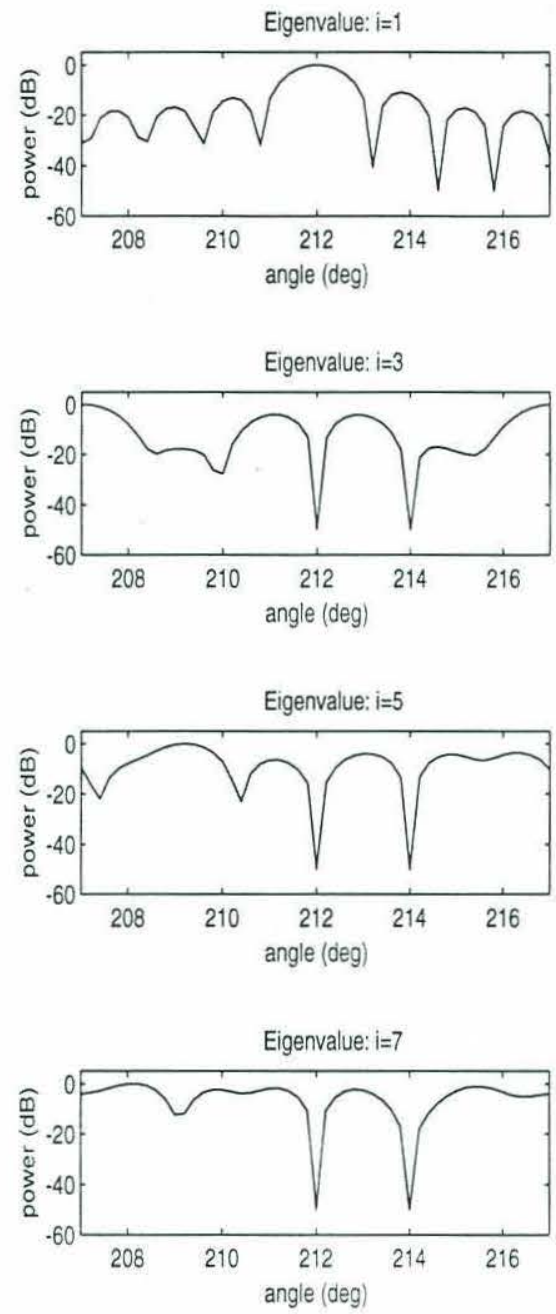

Eigenvalue: $i=2$
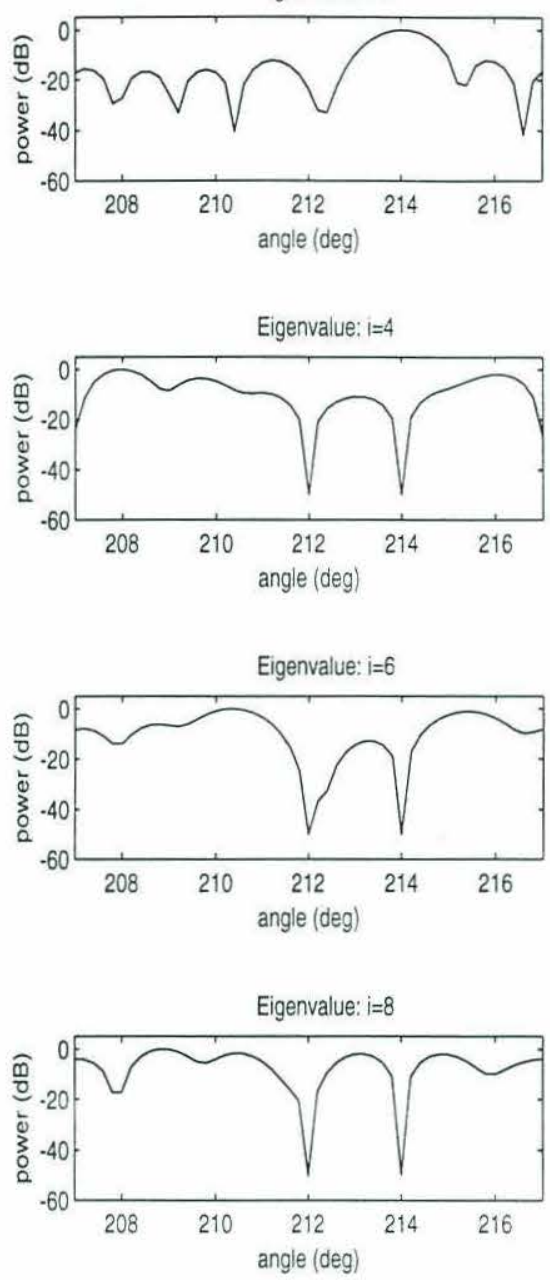

FIGURE A3.18

Eigenvalue Spectrum $\left(\mathrm{N}_{\mathrm{S}}>\mathrm{N}\right)$

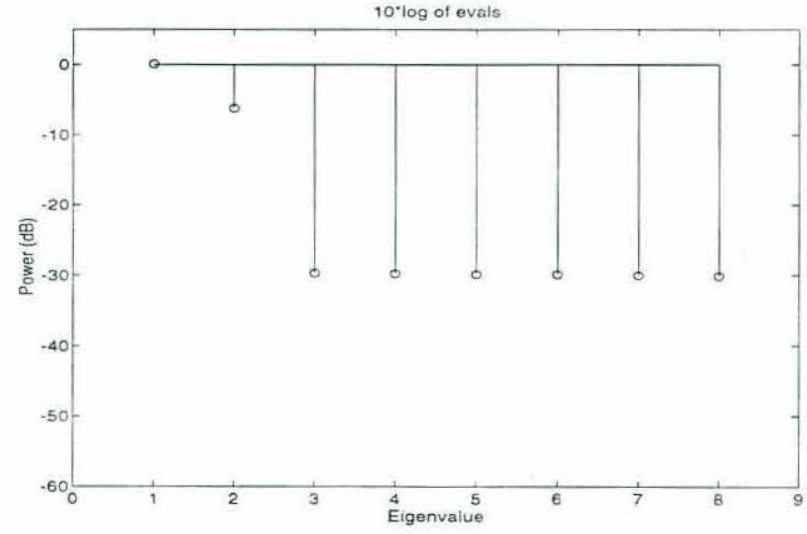


1 / Sum of Weighted Projections: $20 \log _{10}\left(\left[\sum_{i=1}^{k} \frac{\left|d^{H} \cdot \phi_{i}\right|^{2}}{\lambda_{i}}\right]^{-1}\right)\left(N_{S} \gg N\right)$
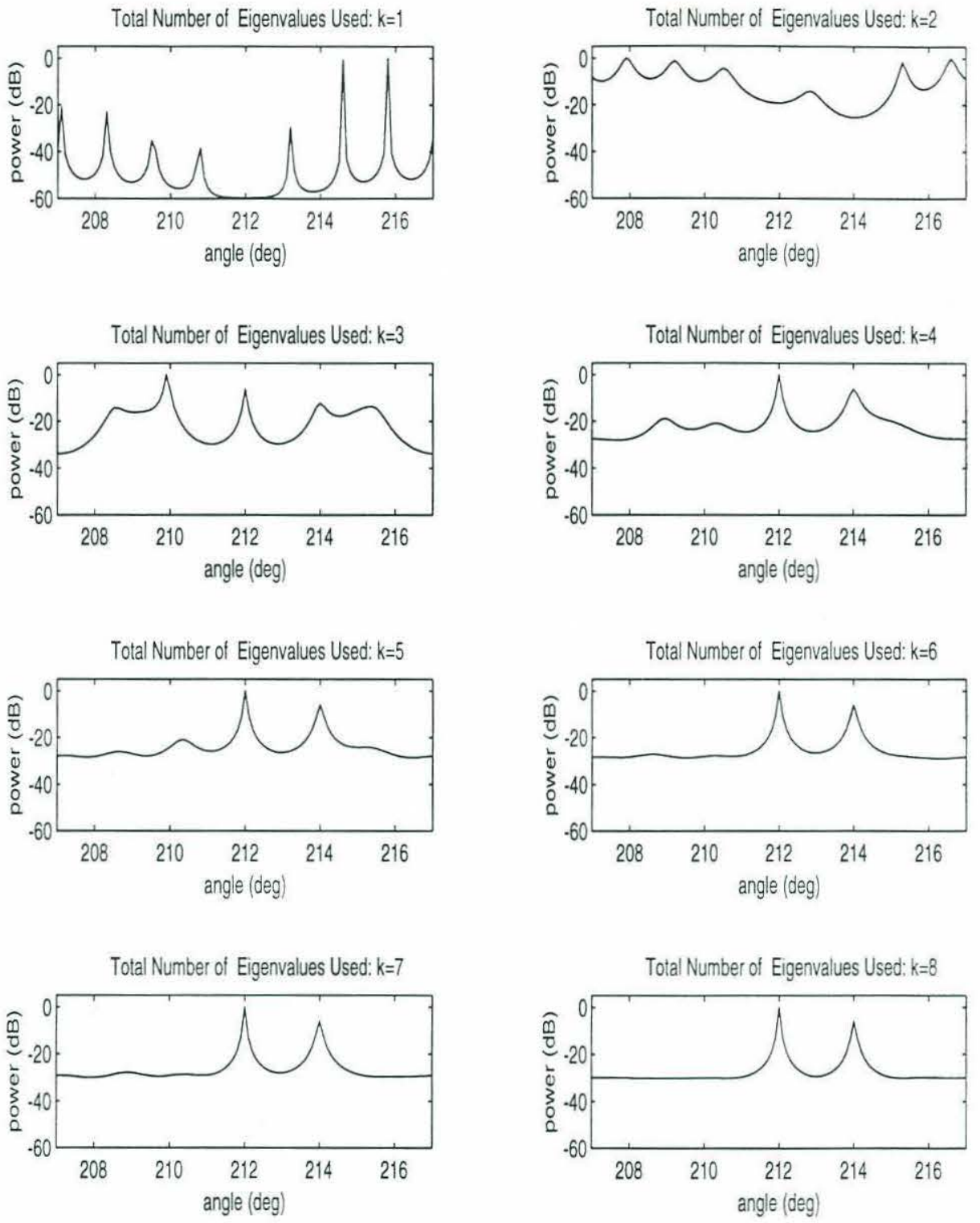

In case 1 with 5000 snapshots the summation involved in forming the sample covariance matrix effectively approaches its expectation value. This can be seen in the eigenvalue spectrum where all the eigenvalues in the noise subspace have magnitude $-30 \mathrm{~dB}$. The response of the MV processor with all 8 eigenvalues contributing accurately captures the noise and signals with no sidelobes. 
Case 2: $($ Number of Snapshots $=8)=($ Number of Channels $=8)$

FIGURE A3.20

Projections of Steering Vector on Eigenvectors: $20 \log _{10}\left(\left|\mathbf{d}^{\mathrm{H}} \cdot \phi_{\mathrm{i}}\right|\right) \quad\left(\mathrm{N}_{\mathrm{S}}=\mathrm{N}\right)$
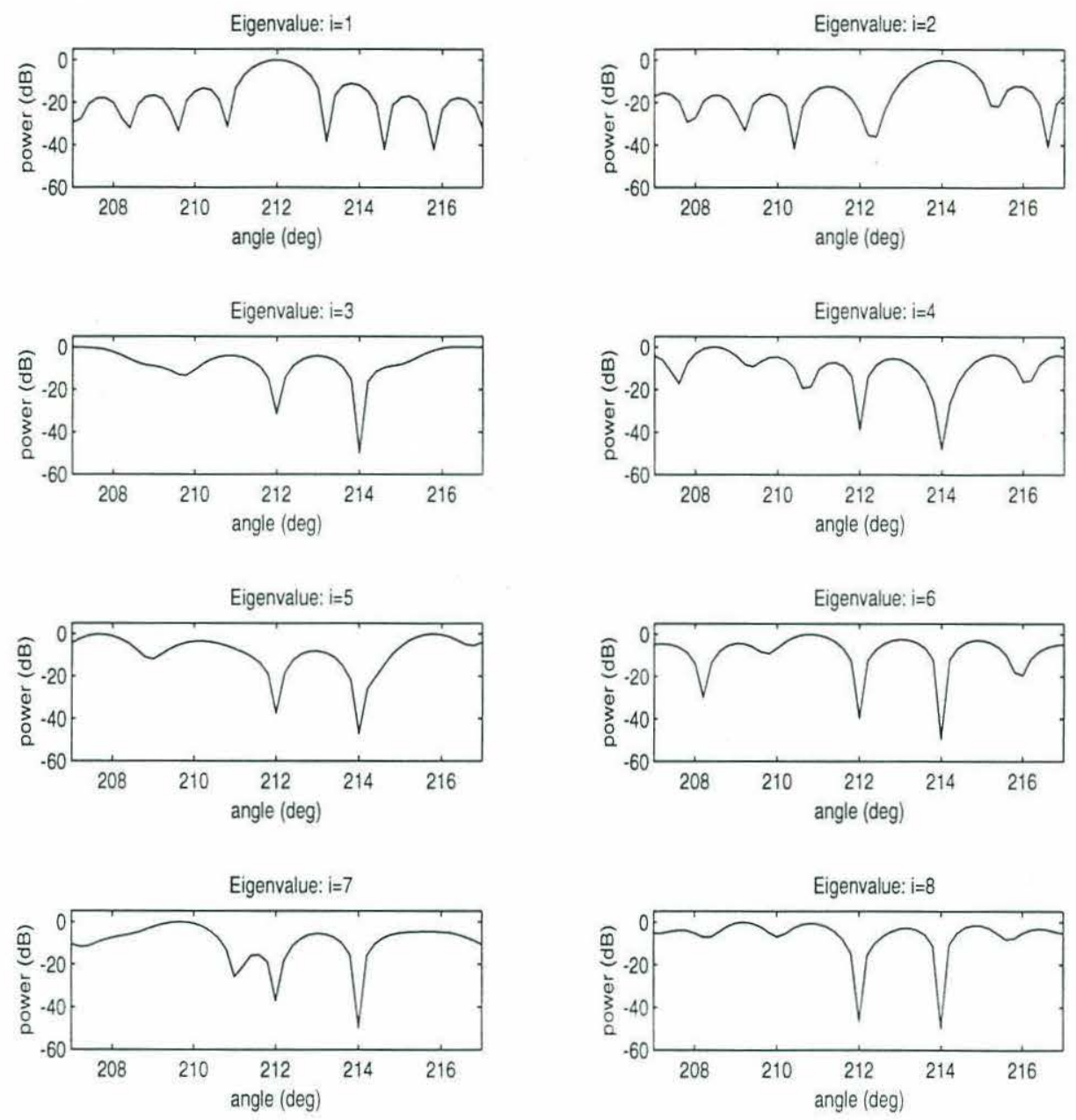

FIGURE A3.21

Eigenvalue Spectrum $\left(N_{S}=N\right)$

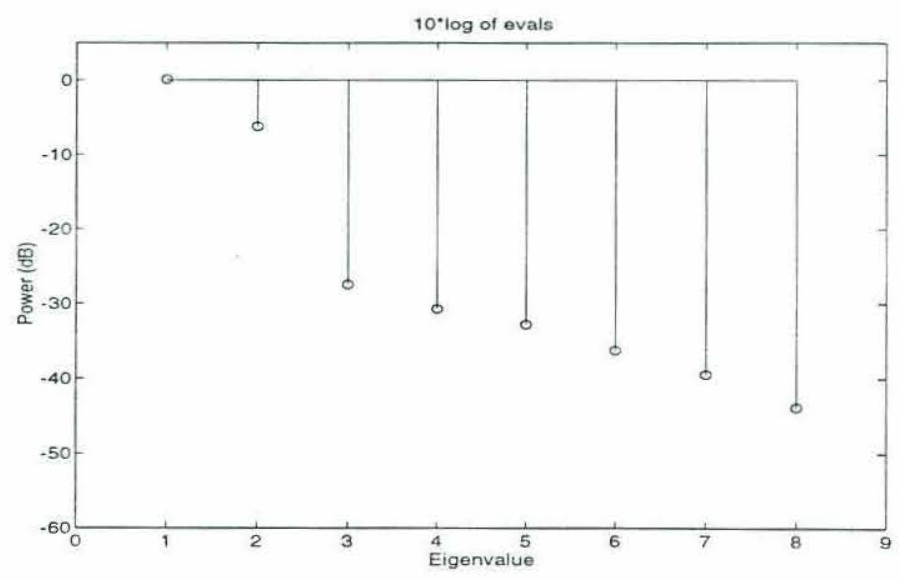


FIGURE A3.22

1 / Sum of Weighted Projections: $20 \log _{10}\left(\left[\sum_{i=1}^{k} \frac{\left|d^{H} \cdot \phi_{i}\right|^{2}}{\lambda_{i}}\right]^{-1}\right)\left(N_{S}=N\right)$

Total Number of Eigenvalues Used: $k=1$

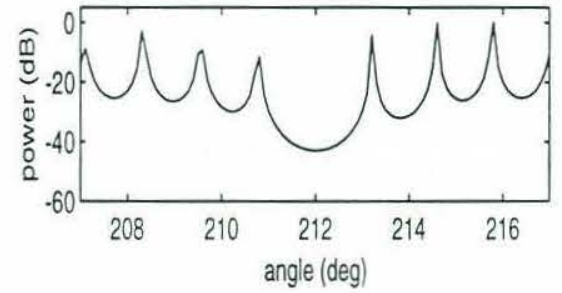

Total Number of Eigenvalues Used: $k=3$

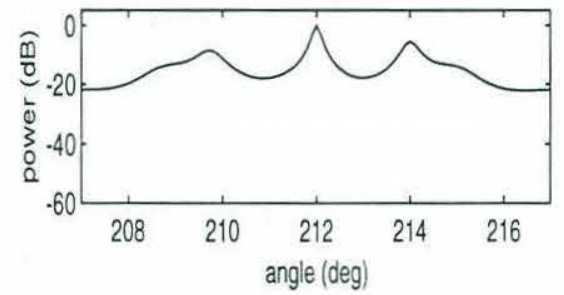

Total Number of Eigenvalues Used: $k=5$

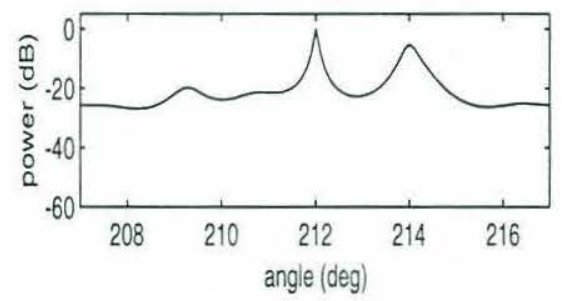

Total Number of Eigenvalues Used: $k=7$

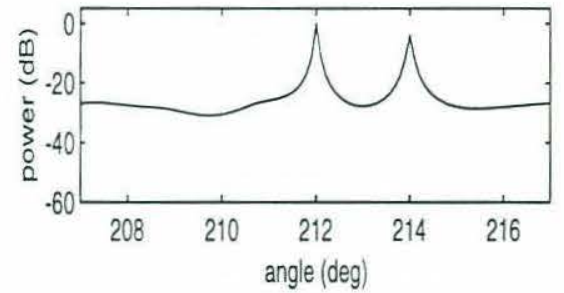

Total Number of Eigenvalues Used: $k=2$

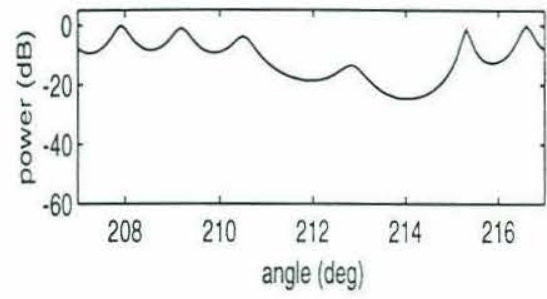

Total Number of Eigenvalues Used: $k=4$

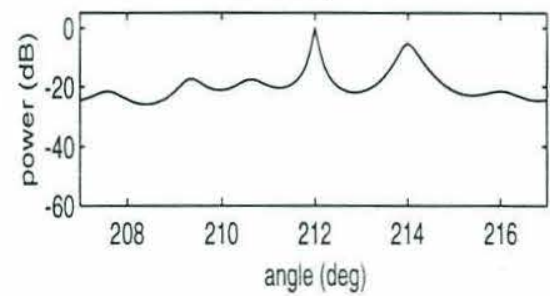

Total Number of Eigenvalues Used: $k=6$

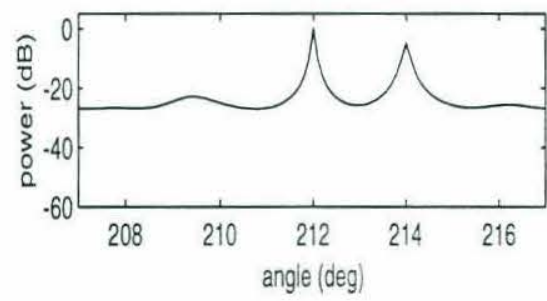

Total Number of Eigenvalues Used: $k=8$

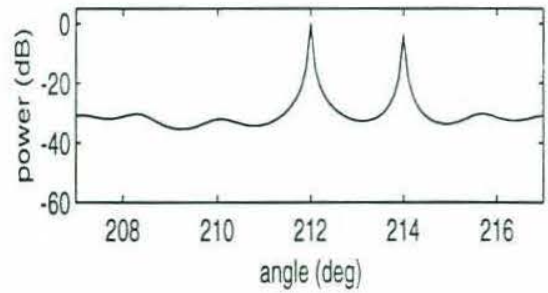

In this case the summation does not converge to its expected value, but it adequately represents the covariance matrix for the purpose of multipath resolution. The eigenvalues in the noise subspace are not completely uncorrelated as some energy is transferred from the lower ones to higher ones, thus giving the unequal spectrum. The effect of this can be seen in the MV response with all 8 eigenvalues where the response has slight peaks away from the signal directions. These "sidelobes" do not present a major problem since they are near the noise level of $-30 \mathrm{~dB}$. 
Case 3: $($ Number of Snapshots $=5)<($ Number of Channels $=8)$

FIGURE A3.23

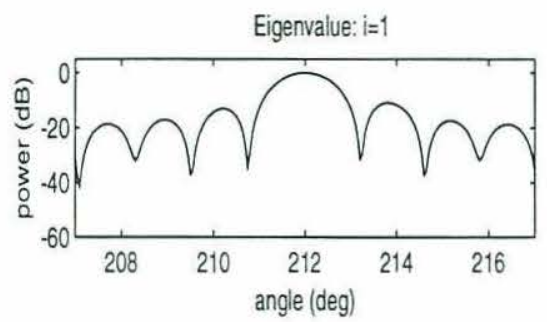

Eigenvalue: $i=3$

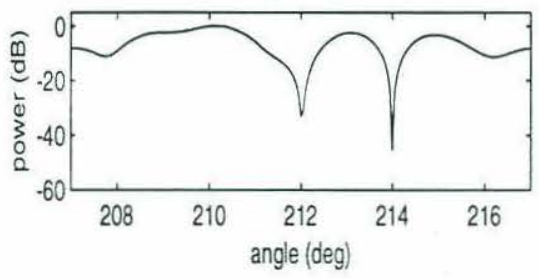

Eigenvalue: $i=5$

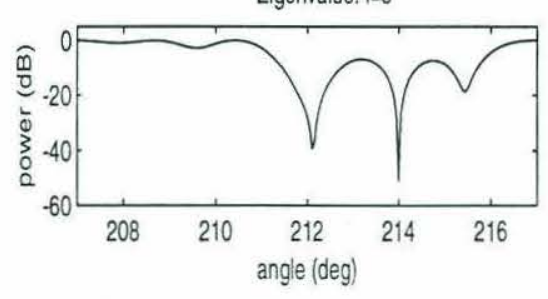

Eigenvalue: $i=7$

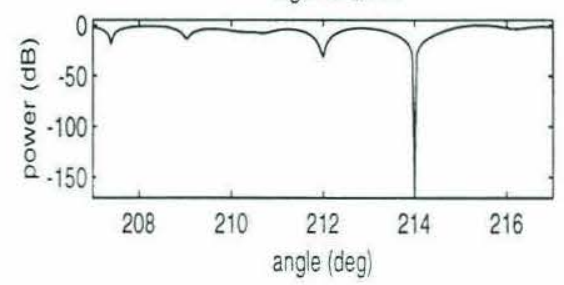

Eigenvalue: $i=2$

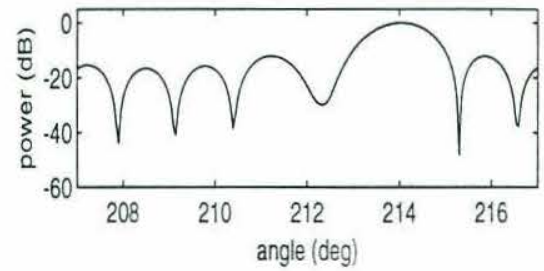

Eigenvalue: $i=4$

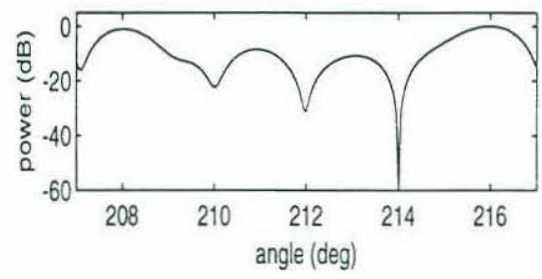

Eigenvalue: $i=6$

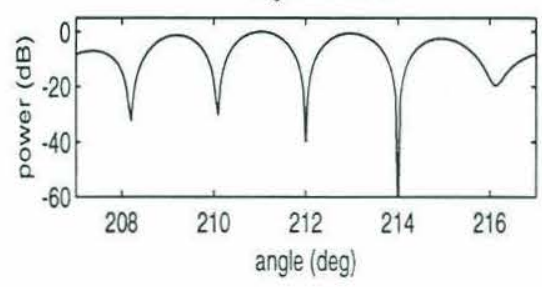

Eigenvalue: $i=8$

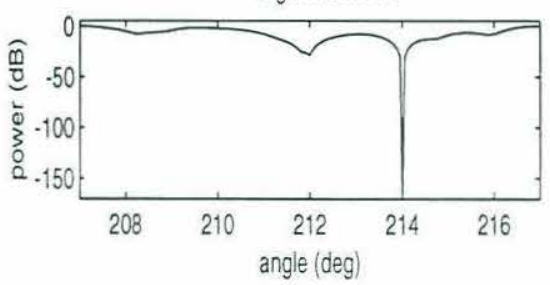

FIGURE A3.24

Eigenvalue Spectrum $\left(\mathrm{N}_{\mathrm{S}}<\mathrm{N}\right)$

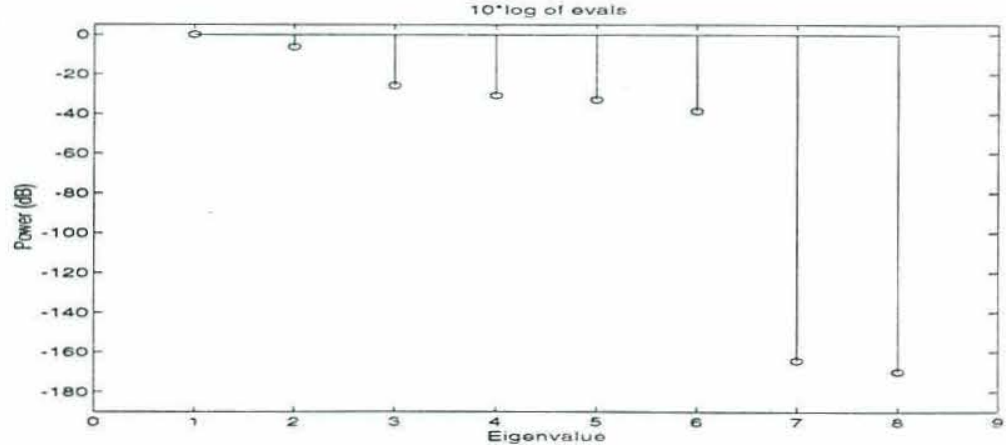




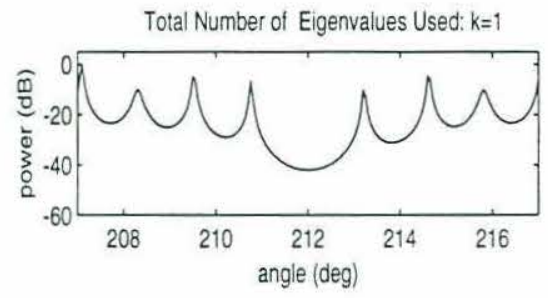

Total Number of Eigenvalues Used: $k=3$

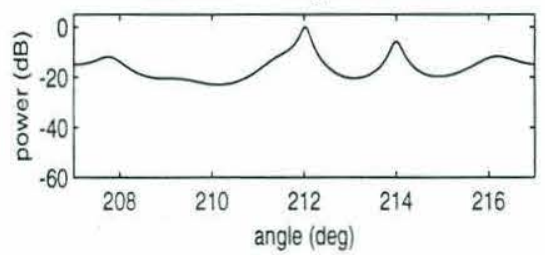

Total Number of Eigenvalues Used: $k=5$

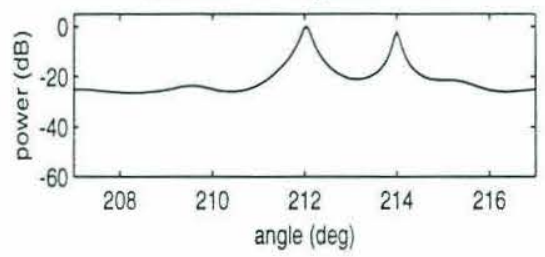

Total Number of Eigenvalues Used: $k=7$

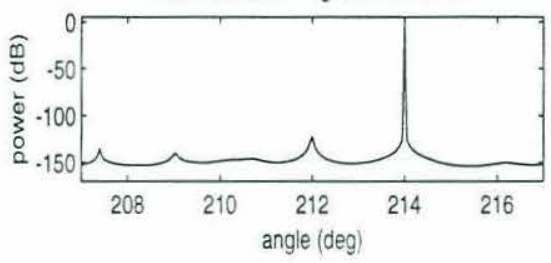

Total Number of Eigenvalues Used: $k=2$

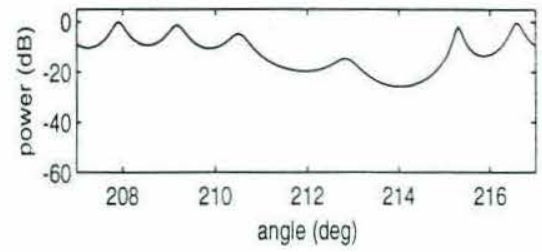

Total Number of Eigenvalues Used: $k=4$

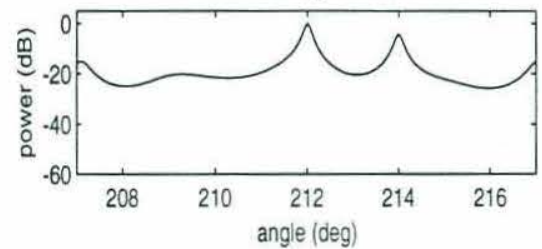

Total Number of Eigenvalues Used: $k=6$

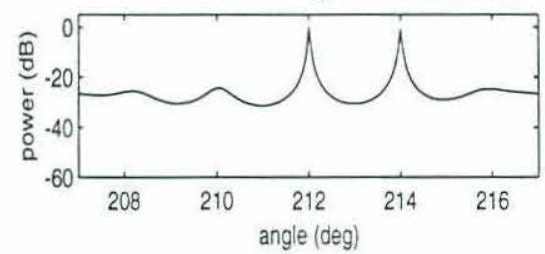

Total Number of Eigenvalues Used: $k=8$

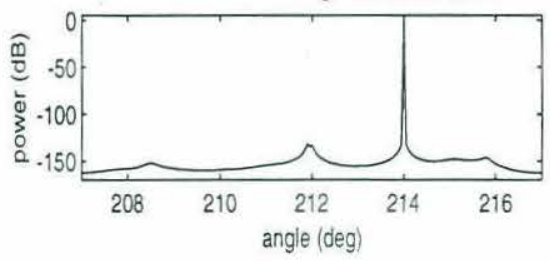

With only five independent snapshots it is impossible to represent the noise in the 8 channel sample covariance matrix. Two of the eigenvalues become effectively zero within numerical precision $(-170 \mathrm{~dB})$. This causes the sum to be dominated by the last two projections. The nulls in these last two projections are very deep since there is no noise in these projections. In the cases 1 and 2 the noise spanned all projections so the nulls could not become overly deep. The results of this summation is seen in the MV response with all eigenvalues where the signal at $214^{\circ}$ dominates. This is clearly detrimental to multipath resolution. The response from the summation with only 5 terms is not dominated by the small eigenvalues since they are not included in the sum. The motivations for eigenvalue decomposition techniques such as MUSIC, or diagonal loading are clearly visible from this example. These techniques are displayed on the following pages. 


\section{Diagonal Loading}

One method of equalizing the eigenvalues is to add a scaled identity matrix to the sample covariance matrix. This also prevents the nulls in the last two projections from becoming overly deep since noise is projected in all dimensions. The response is plotted with the full summation of all eigenvalues and covariance scaling:

$$
\begin{gathered}
\mathbf{K} \rightarrow \mathbf{K}+\sigma^{2} \mathbf{I} \\
\sigma^{2}=\mathrm{sc} \cdot \max (\text { Eigenvalue })
\end{gathered}
$$

\section{FIGURE A3.26}

\section{Case 3:Diagonal Loading Response}

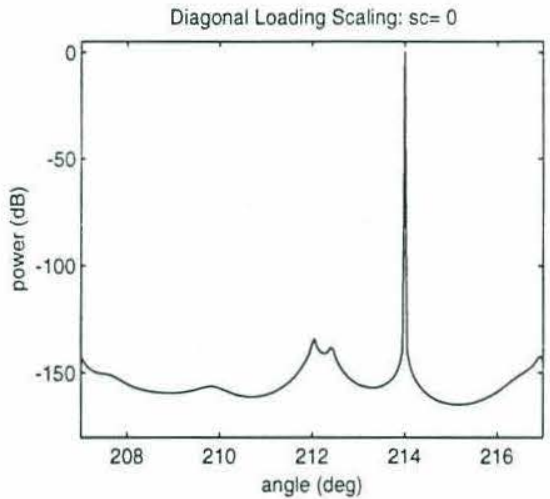

Diagonal Loading Scaling: $\mathrm{SC}=0.01$

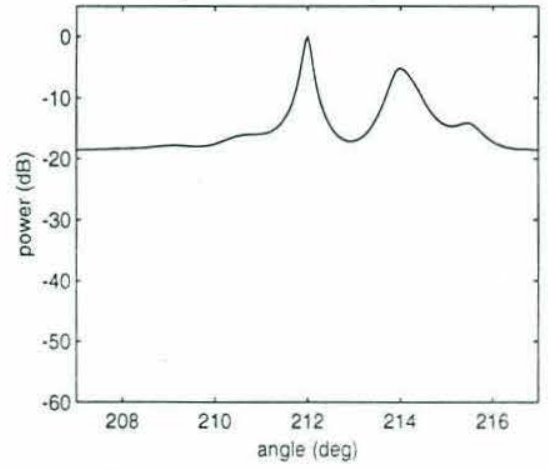

Diagonal Loading Scaling: $\mathrm{SC}=0.001$

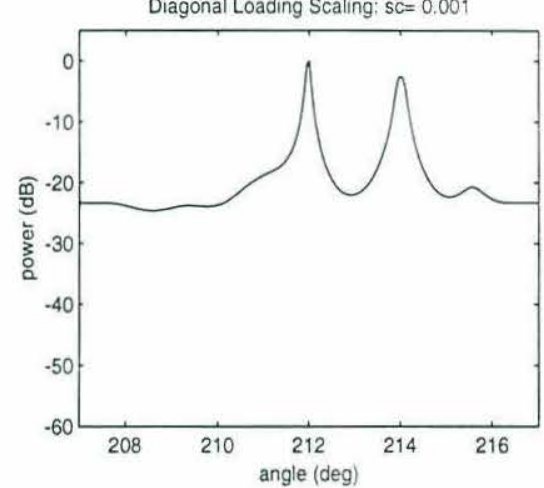

Diagonal Loading Scaling: $\mathrm{SC}=0.1$

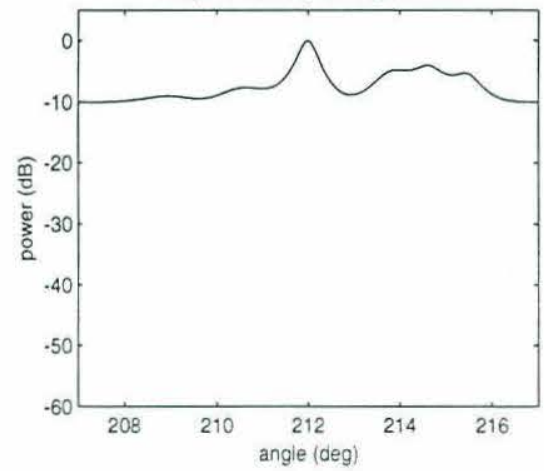

With no diagonal loading the result is the same as the case $3 \mathrm{MV}$ response. With $\mathrm{sc}=0.001,0.01$ acceptable results are obtained. Increasing the scaling to 0.1 causes the noise level to become unacceptably high and resolution decreases. 


\section{MUSIC}

As shown in chapter 3 the MUSIC estimate for power is:

$$
\hat{\mathrm{P}}(\theta)=\left[\sum_{\mathrm{i}=\mathrm{k}+1}^{\mathrm{N}}\left|\mathbf{d}^{\mathrm{H}} \cdot \phi_{\mathrm{i}}\right|^{2}\right]^{-1} .
$$

In this graphic demonstration the number of eigenvalues $(k)$ considered to be in the signal subspace is increased from 1 through 7.

\section{FIGURE A3.27}

\section{Case 3: MUSIC Response}
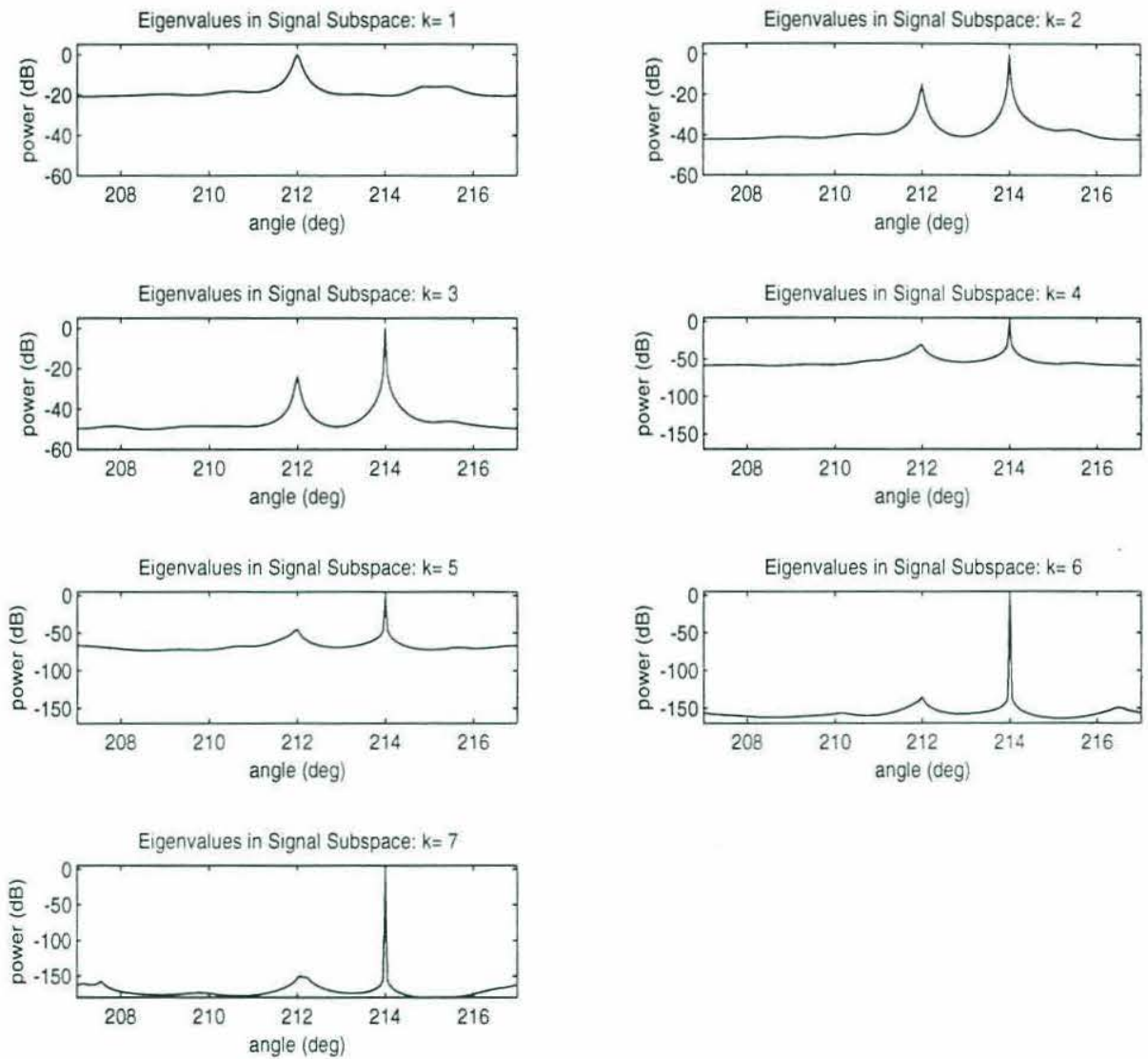

If only one eigenvalue is considered to be in the signal space only one peak occurs since the response is effectively equalized in all other dimensions. The best performance of the MUSIC processor is realized with the signal space chosen to have the same dimension as the number of signals. In this case the performance for resolving the two signals is better than the best case of diagonal loading since MUSIC has a finer resolution as determined by the $-3 \mathrm{~dB}$ points on the mainlobe. This type of performance is demonstrated in figure 3.12 of section 3.4.3. The relative magnitude of the peaks in reversed since the nulls associated with the signal at $214^{\circ}$ are deeper as seen in figure A3.7. If this type of performance is important the diagonal loading approach is more effective. With additional contributions to the signal subspace the performance degrades until with 7 eigenvalues it resembles the MV case 3 response. 



\section{CHAPTER 4}

\section{Directional Spectrum Estimation Results}

In this chapter the methods of chapter 3 are applied to the data received by the CMOAS array during the Heard Island Feasibility Experiment. In figure 3.1 the array is shown to have depth sensors and heading sensors. The mean depth of the array for all stations is approximately $500 \mathrm{~m}$ which is the depth of the sound channel axis at this location. Figure 4.1 shows a sound speed profile from a CTD cast taken in the vicinity of the event 18 station (Heard [1]). A mean sound speed of $\mathrm{C}=1480 \mathrm{~m} / \mathrm{s}$ is used for the beamforming analysis.

\section{FIGURE 4.1}

\section{Sound Speed Profile}

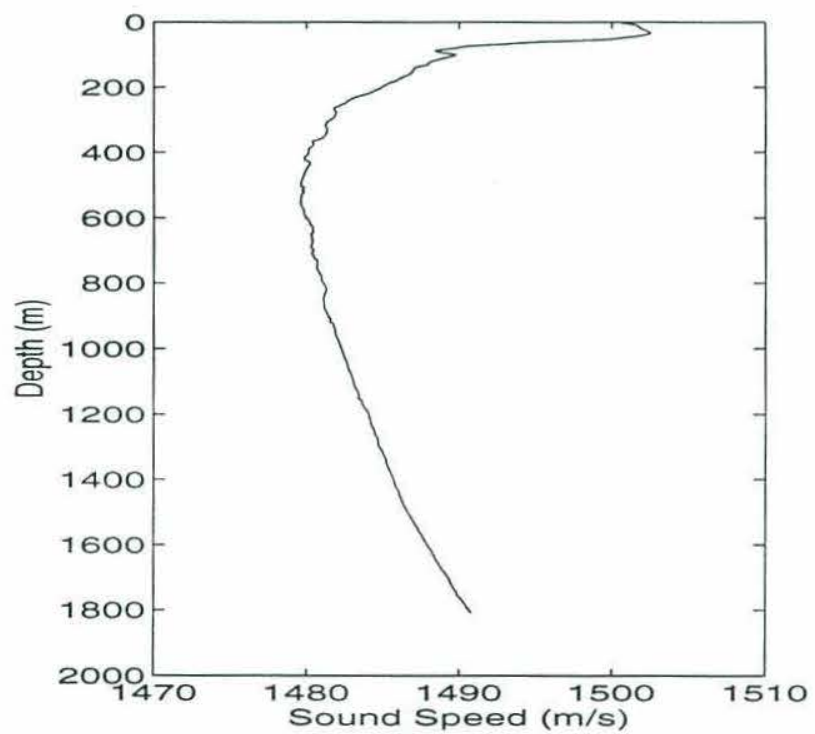

The heading sensors are used to convert the arrival angle recorded with respect to the array to an arrival angle with respect to magnetic north. The details of this conversion are discussed in section 4.4 after the time dependence of array heading is observed on the acoustic data. The arrival angle with respect to magnetic north is then converted to an arrival angle with respect to true north by applying a magnetic declination correction (figure 2.1) to the magnetic heading data. Modelling results and preliminary results indicate an arrival direction of $214^{\circ} \mathrm{T}$ so this is used as the center angle to scan around.(McDonald et al.(1993)[2]) 


\subsection{Conventional medium resolution processing}

Using the MHRA as described in chapter 3 an initial scan of the angular dependence of the incoming power is performed. The Hamming taper is used giving a resolution of $3.23^{\circ}$ and the spatial aliasing lobes are outside the angle space of the beamformer. Sample covariance matrix time windows are $410 \mathrm{sec}$ long with $50 \%$ overlaps. In figure 4.2 and 4.3 contour plots for the time angle dependence of the received power are shown. In figure 4.2 the beamformer is steered from $-90^{\circ}$ to $+90^{\circ}$ of array broadside. This captures the entire angle space of the beamformer aperture since the response is symmetric about the array endfire axis. In figure 4.3 the limited aperture of $214^{\circ} \mathrm{T} \pm 40^{\circ}$ is examined.

These plots show there are no strong secondary signals which enter the main response of the aliased high resolution beamformers.

All plots have the highest power referenced at $0 \mathrm{~dB}$. In all plots there is a strong signal present near the expected arrival angle of $214^{\circ} \mathrm{T}$. The SNR levels are consistent with the one hydrophone results if the signal processing gains of $10 \log _{10}(410 \mathrm{sec}$.) $=26 \mathrm{~dB}$ (for time coherent processing) and $10 \log _{10}(45.7)=16.6 \mathrm{~dB}$ (for white noise array gain with the Hamming taper) are taken into account ${ }^{1}$. The noise field clearly contains directional elements as seen in all of the events, but none of the directional elements are as powerfull enough to infer with the directional spectrum estimation in the aliased beam patterns.

1. The array gain is calculated by: $\frac{\left|\sum_{i=1}^{N} w_{i}\right|^{2}}{\sum_{i=1}^{N}\left|w_{i}\right|^{2}}$, where $w$ is an element of the Hamming weight vector. 


\section{FIGURE 4.2}
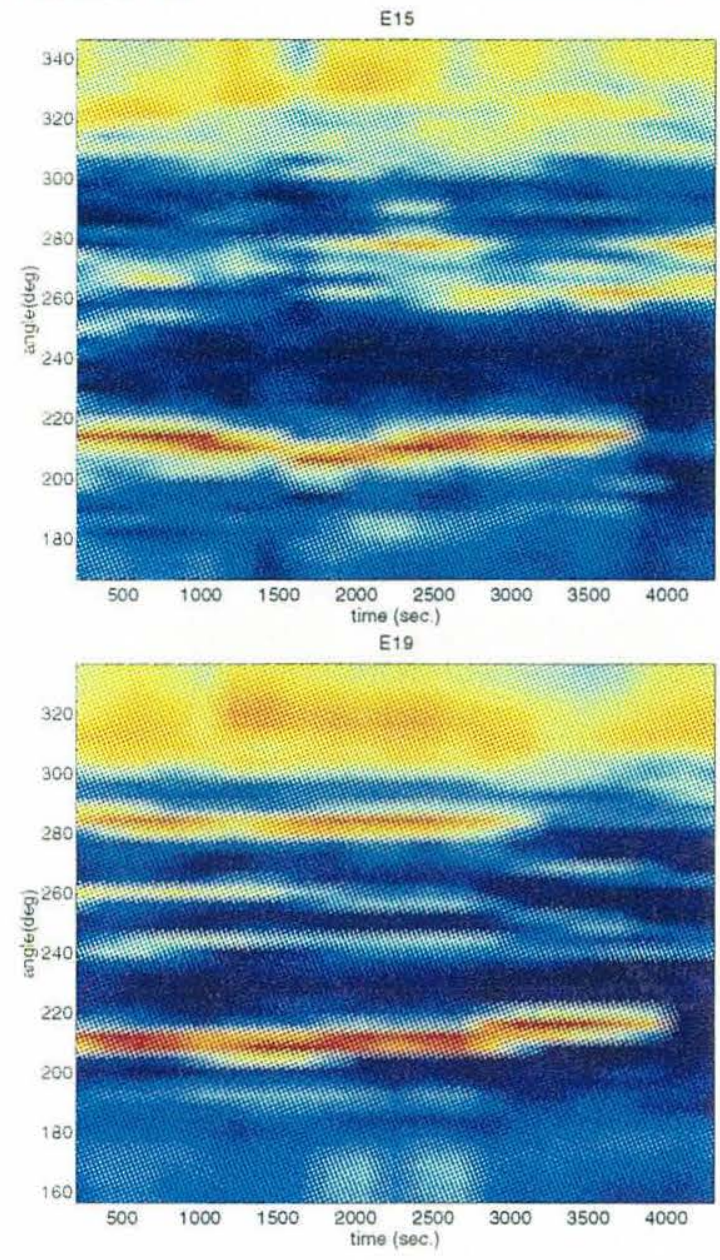
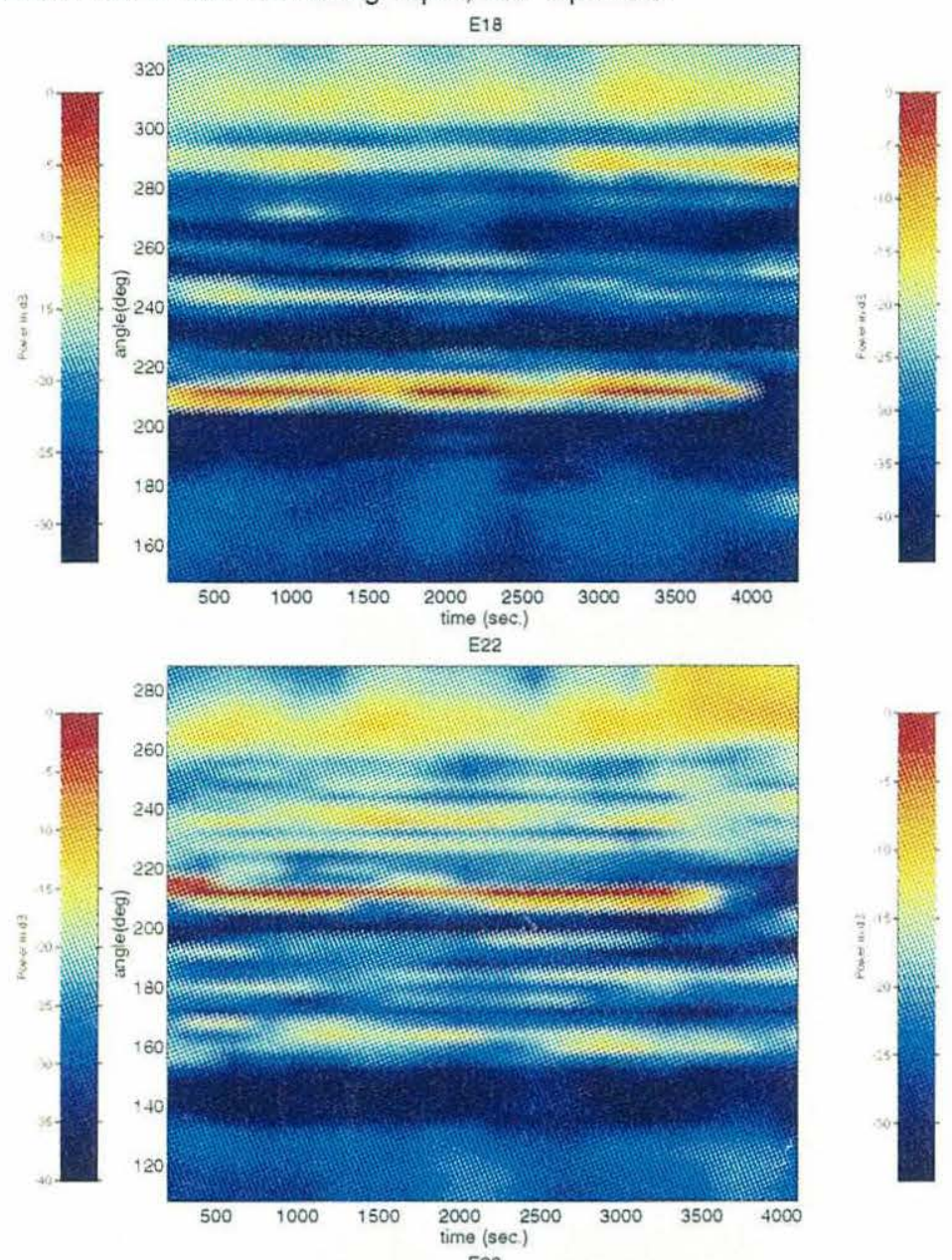

E23
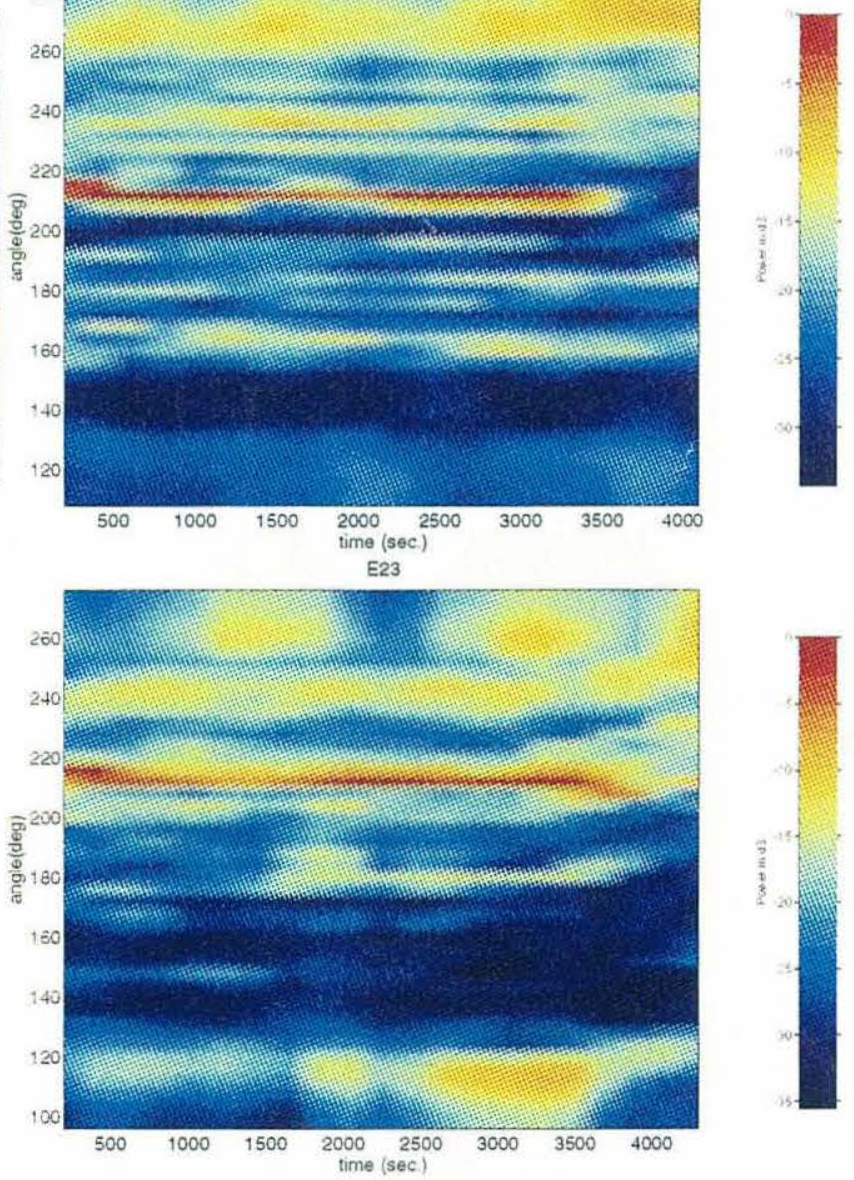


\section{FIGURE 4.3}
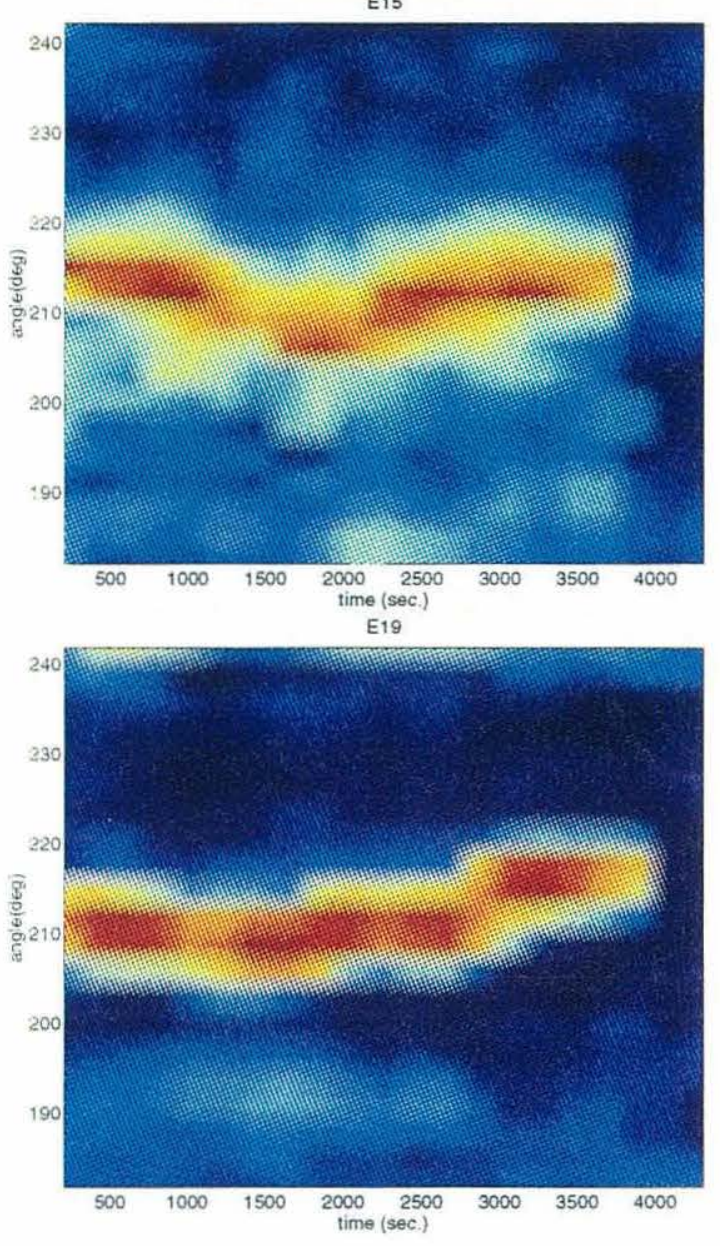

Conventional Processor: CMRA with Hamming Taper, $60^{\circ}$ Aperture

E18
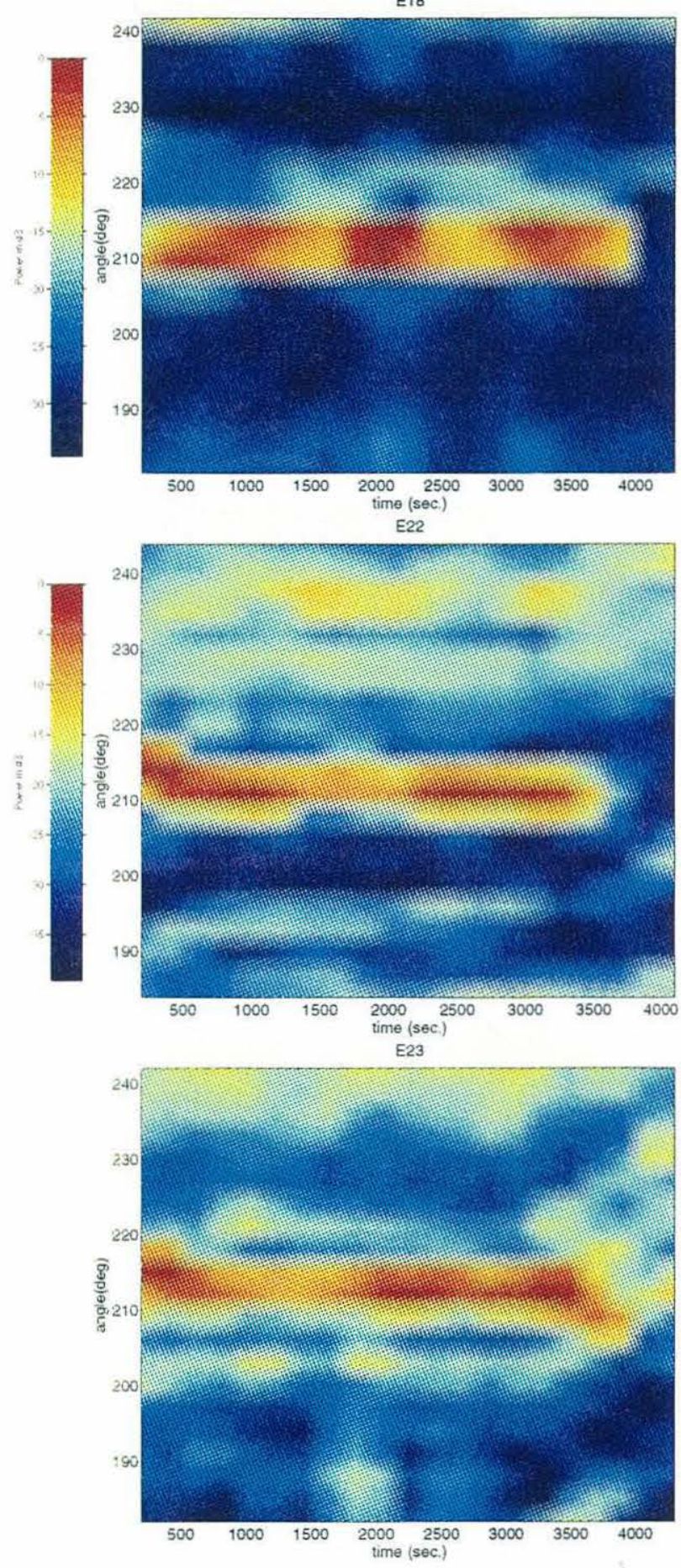
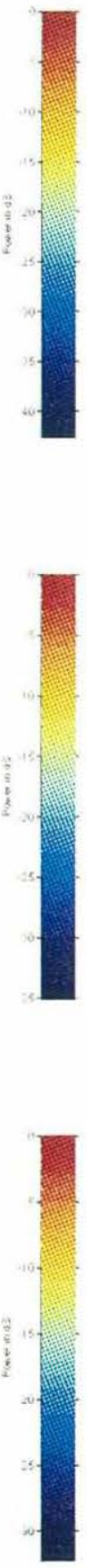


\subsection{Conventional high resolution processing}

The CHRA is used to zoom in on the signal peak of event 15. A Hamming taper is used giving a resolution of $1.6^{\circ}$ and sidelobes of $-41.8 \mathrm{~dB}$ as shown in chapter 3 . The spatial aliasing distance is now $43^{\circ}$, but the aliasing is not visible in the limited angular region of $213^{\circ} \mathrm{T} \pm 8^{\circ}$ that is scanned. Only one pentaline event is examined. The angular power of the pentaline sidebands is compared to the center band in figure 4.4. The angle scale on the $y$ axis of this figure is $\theta_{\text {steer (time averaged) }}$ as is explained in section 4.3.1.

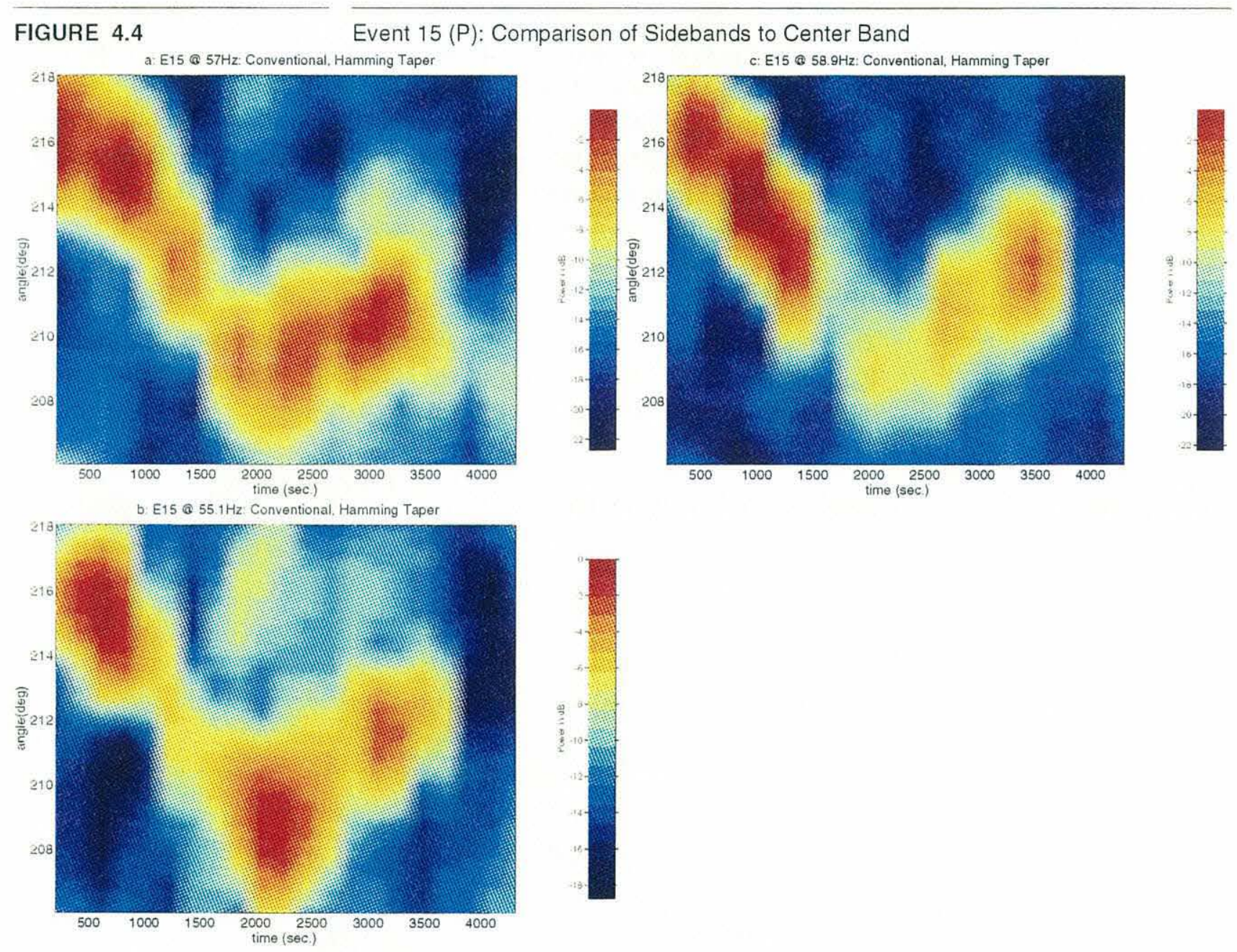

The sidebands are very similar to the main band in time dependent directional characteristics, but the amplitude fluctuates independently for the three signals. The higher resolution of the CHRA with a Hamming taper does not reveal any multipath effects. Only one strong signal is present in the plots. The most dominant feature of these plots is the apparent change of direction of the incoming signal vs. time on all bands. This can be attributed to variations in array direction. 


\subsection{Compensating for array geometry variations}

During the course of the trial the array was not quite straight behind the towing ship. As seen in figure 4.5 there are three heading sensors along the array. These sensors record the heading of three points on the array sampled every fifteen minutes. Initial examination of the data from these sensors reveal that the array curves slightly as there are differences of up to four degrees in adjacent heading sensor readings at an instant in time. More significantly, the array is turning as a function of time as temporal differences in a single array heading sensor are as large as eight degrees over a one hour reception period. Consequently, two approaches are used to deal with the problem of the variable array geometry. In the first approach, the array is modelled as a straight line that can change angle as a function of time as shown by the heavy line in figure 4.5. This is adequate for conventional processing methods and is used to determine absolute reception angles. The second approach incorporates the curvature of the array as it changes with time, but does not track a reference direction as in the first method. This is used for adaptive processing which is very sensitive to array geometry.

\subsubsection{Array turning}

The contour plots of figure 4.3 are in the reference frame of the array. Thus if the angle $\theta_{\text {array }}$ changes, and the signal does not change its direction with respect to true north, the direction of the signal relative to the array $\left(\theta_{\text {relative }}\right)$ changes.

\section{FIGURE 4.5}

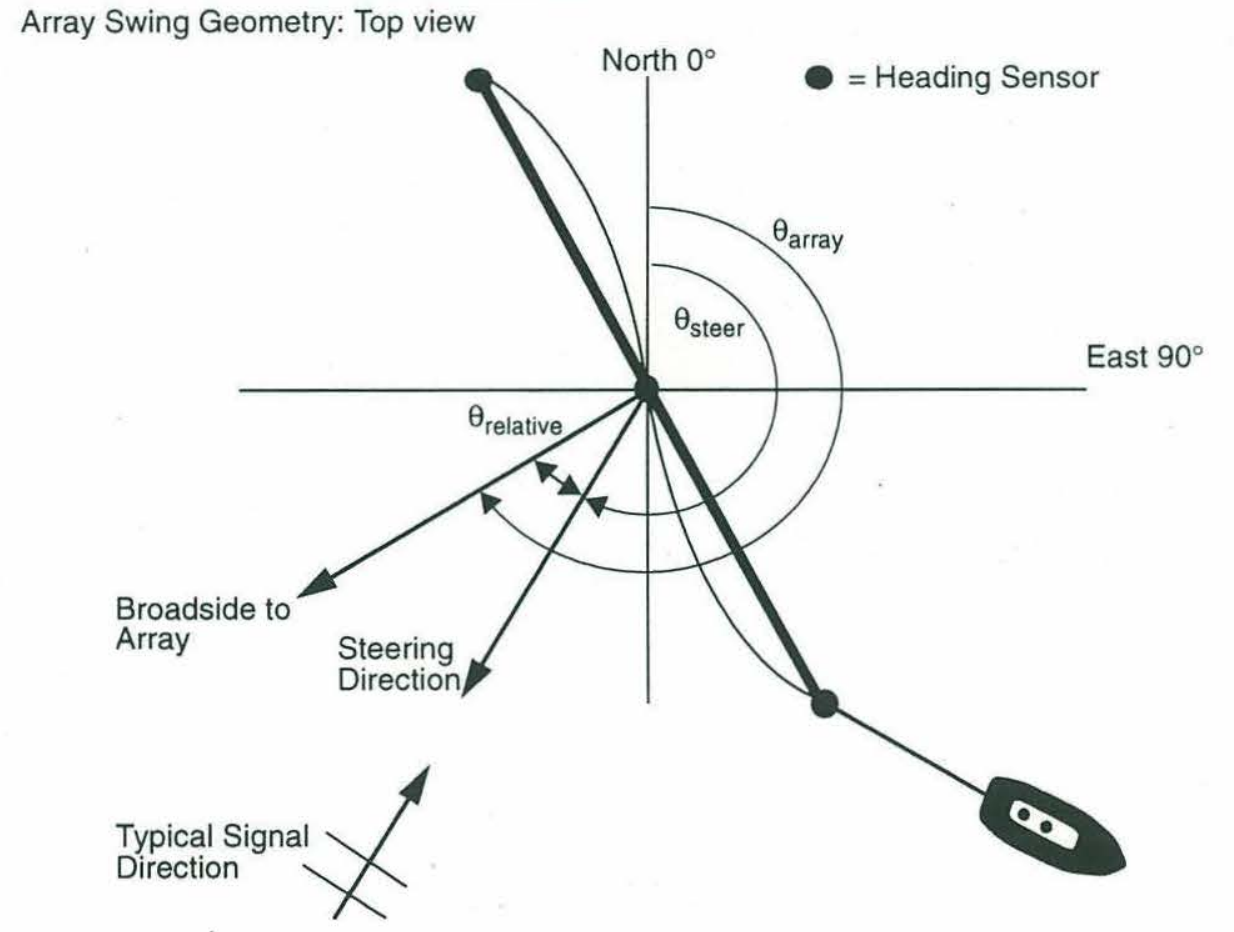

The heavy line in figure 4.5 shows the array's broadside direction $\theta_{\text {array }}$ with respect to true north while the lighter curved line below is a typical actual array geometry. The straight line array direction is determined by a weighted average of the three heading 
sensors. The weights are calculated from the distance of the sensor along the array.

Seven time samples of the array heading spanning the $1 \mathrm{hr} .15 \mathrm{~min}$. data records are then interpolated in time using a cubic spline interpolation algorithm to give a array heading at the center of each time window in which a covariance matrix is formed. These inter-

polated time samples of the array direction are denoted $\theta_{\text {array (time dependent) }}$. For the typical case of $410 \mathrm{sec}$. windows with $1 / 2$ overlap this results in 21 interpolated time samples. The time dependent array heading is expressed as a time averaged mean component plus a time dependent quantity fluctuating about the mean.

$$
\theta_{\text {array(time dependent) }}=\theta_{\text {array (time averaged) }}+\theta_{\text {array (fluctuating) }}
$$

The mean of these interpolated time samples forms a reference direction for the array, which is used as the time averaged array heading for the plots of figure 4.3. The beamforming processor can only compute the angle relative to the array broadside ( $\left.\theta_{\text {relative }}\right)$. The $y$-axis scale in figures 4.3 is determined by adding the $\theta_{\text {relative }}$ to the time averaged array heading reference.

$$
\theta_{\text {steer(time averaged) }}=\theta_{\text {relative }}+\theta_{\text {array (time averaged) }}
$$

Figure 4.6 shows the array coordinate system changing as a function of time. The fluctuations of the array heading with respect to $\theta_{\text {steer(time averaged })}=213^{\circ} \mathrm{T}$ are plotted as a green line. The points used to interpolate this line are shown as yellow circles. Constants are added to these lines to create white grid lines that represent the time varying coordinate system of the array. The quantity plotted in these lines is

$$
\theta_{\text {steer(time dependent) }}=\theta_{\text {steer(time averaged) }}+\theta_{\text {array (fluctuating) }}
$$

Combining equations $4.1,4.2$ and 4.3 the grid lines become

$$
\theta_{\text {steer(time dependent })}=\theta_{\text {relative }}+\theta_{\text {array (time dependent })} .
$$

The $\theta_{\text {steer(time dependent) }}$ are originally plotted with $\theta_{\text {steer(time averaged) }}$ as the $y$-axis labels. These labels are replaced in the plotting routine by labels that mark the fluctuating line with the time averaged steering angles $\left(\theta_{\text {steer(time averaged })}\right)$ they are fluctuating about. This is necessary since the $\theta_{\text {array(fluctuating) }}$ is not zero at time zero.

This procedure captures the time varying nature of the coordinate system referenced to $\theta_{\text {steer(time dependent) }}$ in which the array steers. In a normal contour plot one would look at the $y$ axis scale and then look directly across to see the power at that angle as a function of time. In the plots of figure 4.6 the $y$-axis scale is labelling the curved white grid lines. Thus to see the power at say $213^{\circ} \mathrm{T}$, one should follow the green line across the plot. 
$\overline{-100}$ 
FIGURE 4.6
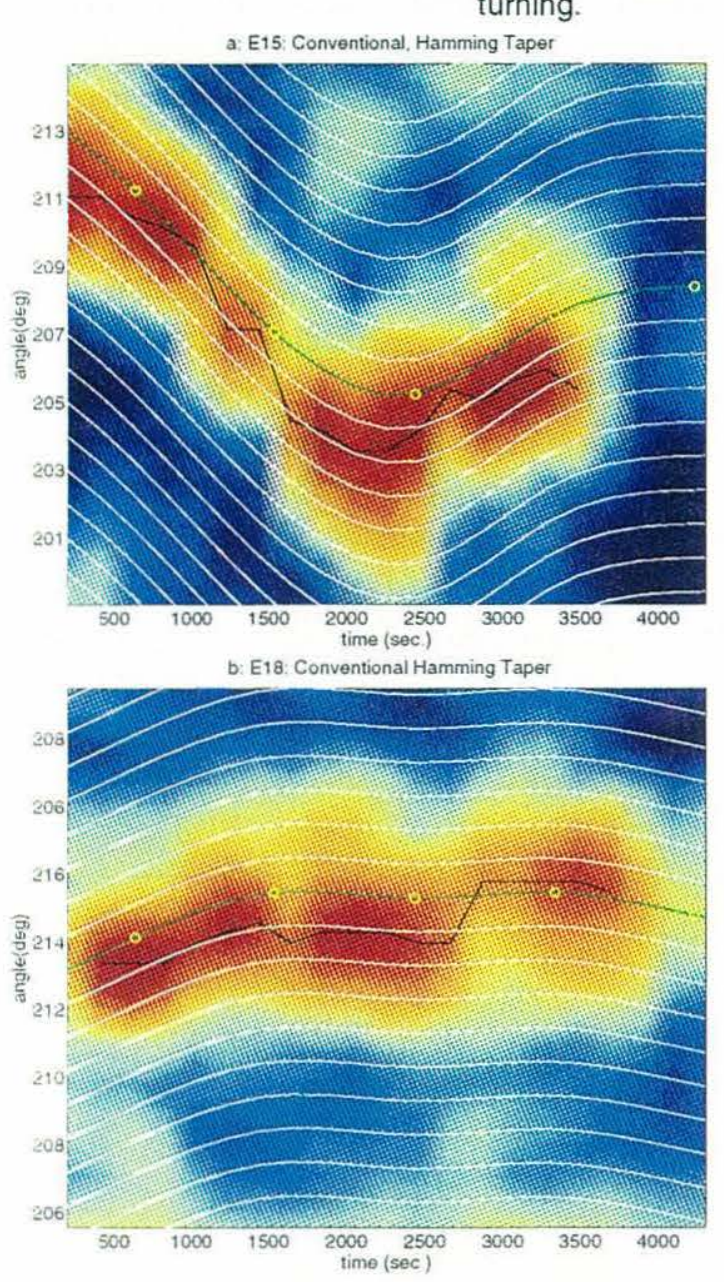

C: E19: Conventional, Hamming Taper

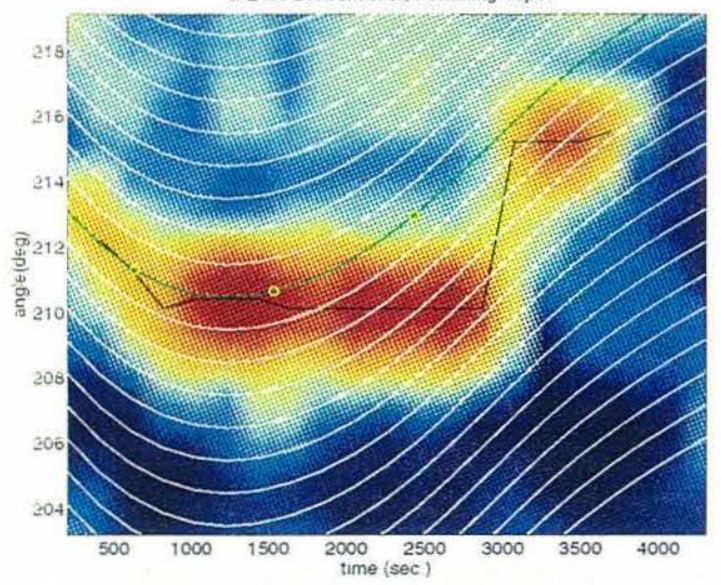

d E22, Conventional, Hamming Taper
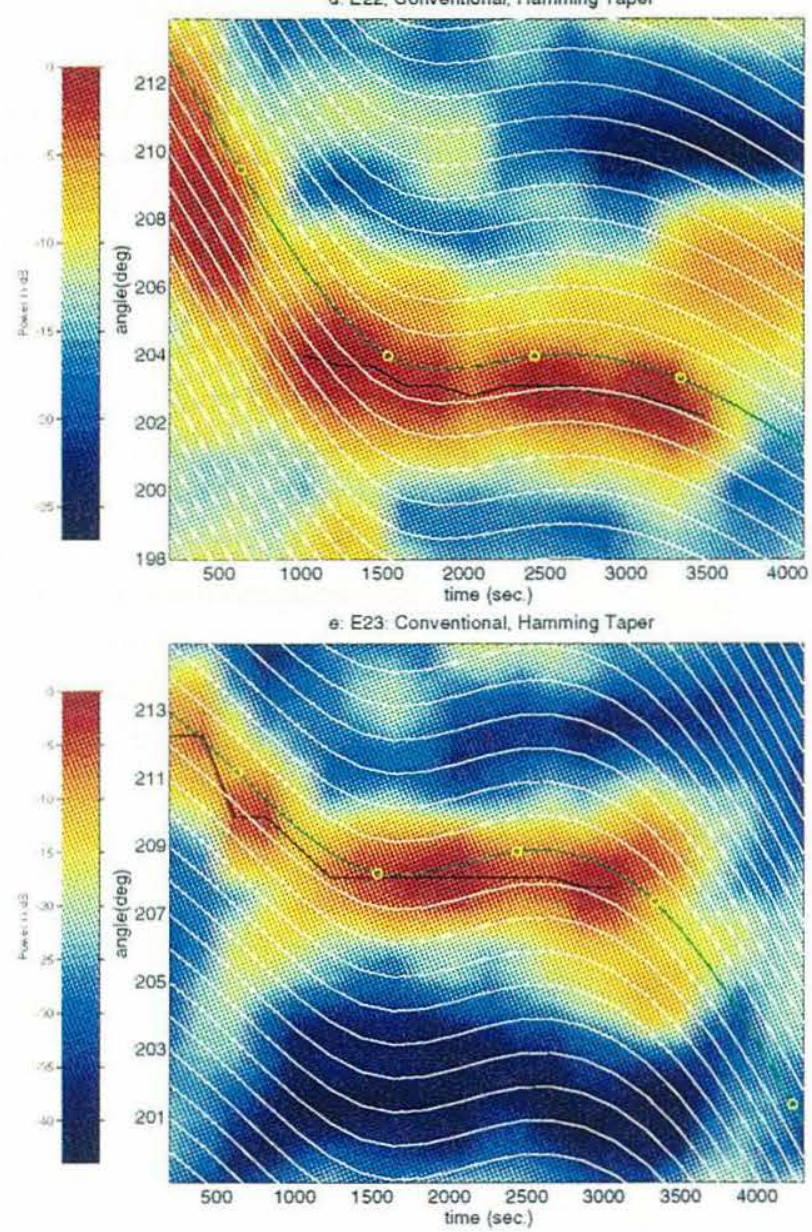
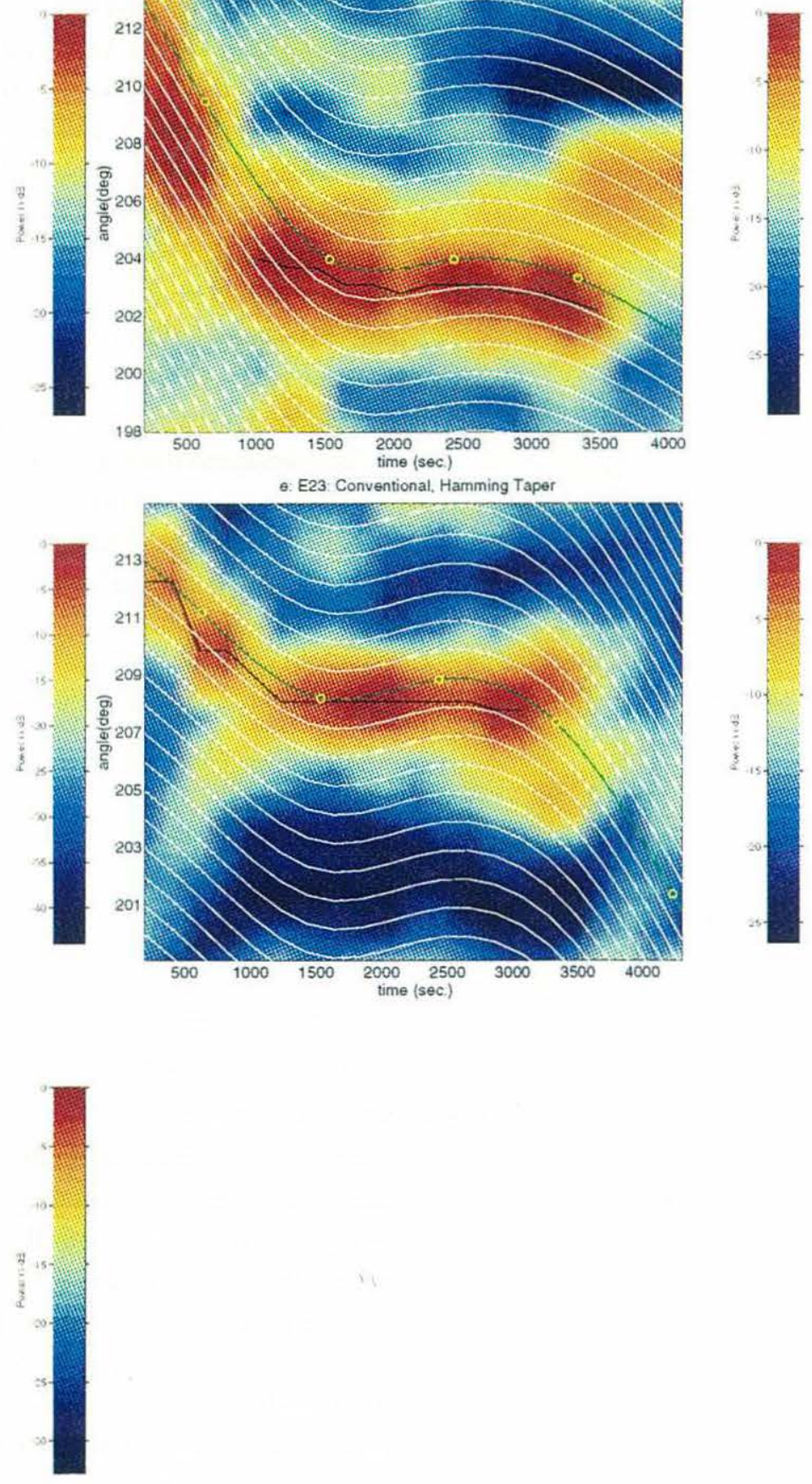


\subsubsection{Absolute arrival angles and error estimates}

As seen in figure 4.6 the grid lines track the signal direction fluctuations fairly well on all events within the limitations of the infrequently time sampled array heading data and the 410 second time windows used to form covariance matrices. To calculate an absolute mean arrival angle for the signal, the difference in angle between the peak incident power (black line on figure 4.6) and the grid line closest to this line is averaged over the time of signal reception. This difference is then added to the referenced grid line to give an absolute angle. The arrival angles, as determined by the above method and the conventional beamformer with a uniform taper for all events are tabulated in table 4.3. The standard deviation of these measurements is probably not a good estimate of the accuracy, but it does show remarkable consistency between events after the turning corrections have been performed. A better estimate of the error in these measurements is to examine how well the signal direction tracks with the array direction in figure 4.6. This is performed visually by comparing the black peak power direction line to the white rotating reference frame grid lines. From this it is seen that the black line remains within 1.5 degrees of its mean direction for all events except when the array is turning rapidly . Only time segments when the array is not turning rapidly, as seen by the turning index of section 4.3.4, are used to estimate arrival angles.

TABLE 4.3

$\begin{array}{ll}\text { Event Name } & \begin{array}{l}\text { Arrival Angle } \\ \text { Degrees from True North }\end{array}\end{array}$

\begin{tabular}{lll} 
Event 15 & 211.9 \\
Event 18 & 212.2 & \\
Event 19 & 211.7 & \\
Event 22 & 212.0 & \\
Event 23 & 212.3 & \\
\hline Mean & 212.0 & Standard Deviation $=0.24$
\end{tabular}

\subsubsection{Angle corrections}

There are two approaches to correct for the swinging of the array in angle. The first is to use the array heading data and subtract out the fluctuations. This approach effectively straightens out the white grid lines in the plots of figure 4.6. The second approach is to assume that the signal should come from a constant direction in time and that any changes are due to varying array geometry. This approach assumes the array heading data, although qualitatively correct, is not as accurate for correcting the direction as the signal itself. The signal can be straightened out by subtracting the fluctuations in the peak power direction, and effectively straightening the black lines in figure 4.6 . The second approach is used for displaying the results of the adaptive processors. The peak power direction is straightened along the absolute arrival angle direction, determined by the methods of the previous section. 


\subsubsection{Array curvature}

As mentioned previously, initial examination of the array heading data shows differences of zero to four degrees between sensors at a given instant in time. If a simple model for array curvature is used based on arcs as shown in figure 4.7 , then a difference in array heading of three degrees between sensors causes a maximum array displacement of 15.7 meters, which is more than $1 / 2$ the wavelength of $26 \mathrm{~m}$ at $57 \mathrm{~Hz}$. According to the sensitivity analysis to array curvature performed in section 3.5 , this is clearly significant especially with the adaptive processors

FIGURE 4.7

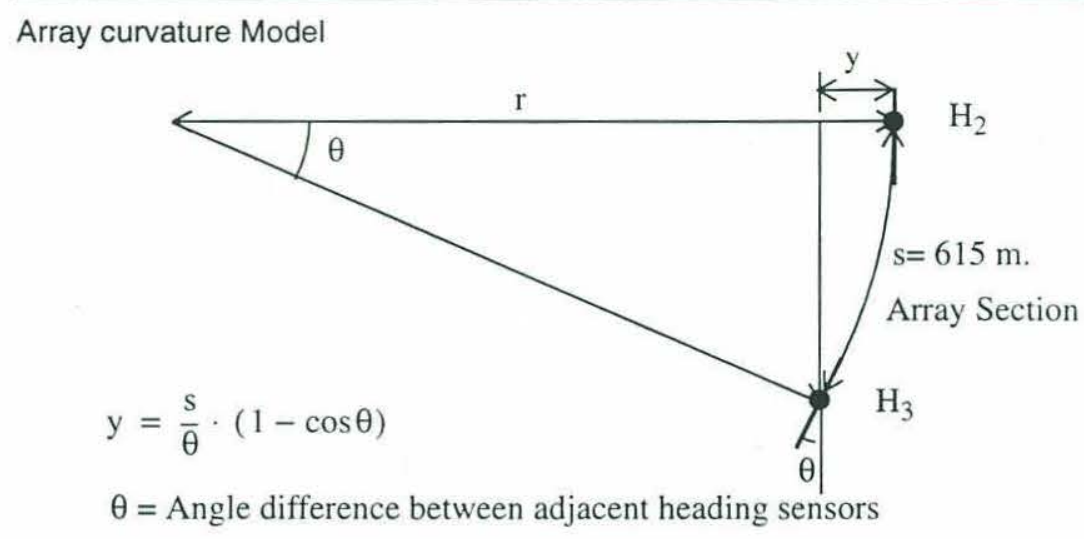

To examine the effects of the array curvature on the beamforming results, a crude index for the amount of curvature is used. In figure 4.8 the standard deviation of the three heading sensors is plotted vs. time for each event. One unit of the curvature index corresponds to approximately $1 / 5$ of a wavelength amplitude curvature at $57 \mathrm{~Hz}$. The results of section 3.5 show the adaptive processor performance degrading after $1 / 8$ of a wavelength array curvature. The actual performance of the adaptive processors is adequate at array curvature indices of one or slightly greater as seen in events 18 and 19. Thus the curvature index may exaggerate the actual amount of curvature, but it does give a qualitative sense of performance. In event 22 where the curvature index is as high as three or four the adaptive performance is poor, as seen in figures 4.9 through 4.13 .

Figure 4.8 justifies using the straight line model for events 18 and 19. It also shows that good results should not be expected at the beginning of event 22 . Even if the array curvature is cancelled correctly here, the array is turning (as seen by the array turning index) quickly enough so that the angle of the received signal with respect to the array is a non-stationary process. The array turning index is the derivative of the straight line array direction with respect to time, normalized by an arbitrary constant (450) to fit on the same scale as the array curvature index.

$$
\text { Array Turning Index }=\frac{\Delta \mathrm{H}(\text { deg. })}{\Delta \mathrm{t}(\mathrm{sec} .)} \cdot 450
$$

Figure 4.8 also shows that when the array has high curvature it tends to be turning rapidly. This makes sense if the array is thought to "slide" through the water. 
Array Curvature and Turning Indices
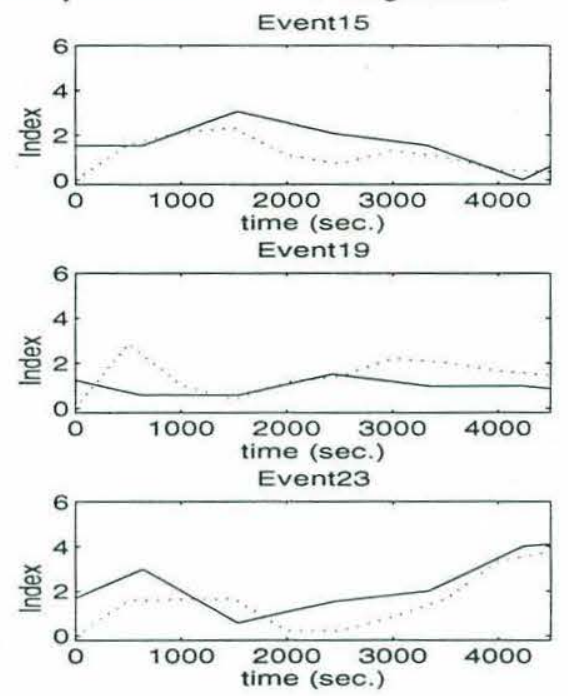
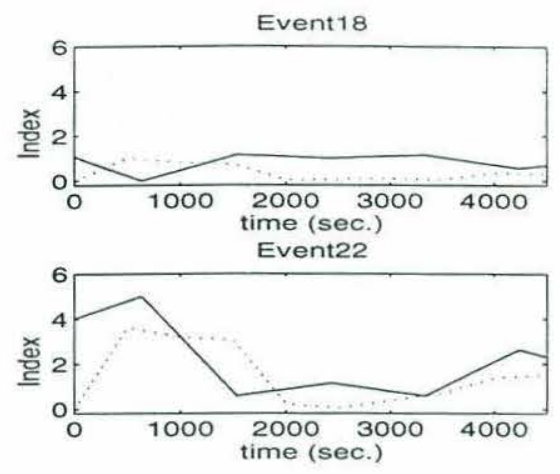

Curvature Index: -

Turning Index:

\subsection{High resolution adaptive processing}

\subsubsection{Effects of diagonal loading}

In chapter 3, section 3.4.1.4, a technique of stabilizing the inversion of the covariance matrix by adding a small amount of band-limited white noise is mentioned. This can be accomplished by adding an identity matrix scaled by a percentage (s) of the most powerful eigenvalue. In section 3.4.1.3 the covariance matrix order is reduced by spatial prefiltering on subarrays to have the same order $(\mathrm{N}=8)$ as the number of independent data snapshots used in forming the covariance matrix. The number of independent data snapshots or the number of uncorrelated signals incident on the array, whichever is greater, determines the rank of the covariance matrix. Thus assuming there are enough uncorrelated signals, which is a safe assumption given the ocean noise background as shown in section 4.1, the covariance matrix is full rank. Since the covariance matrix is full rank the inverse is stable and diagonal loading is not required. Diagonal loading effects are demonstrated in figure 4.9 on event 19. It is seen that diagonal loading decreases the resolution of the MV processor which is not a desired effect.

If the prefiltering order reduction operation is not used, then the order of the covariance matrix $(\mathrm{N}=32)$ is greater than the number of snapshots (8) given the time bandwidth product of the windows. In this case, the covariance matrix is singular and diagonal loading is required. 


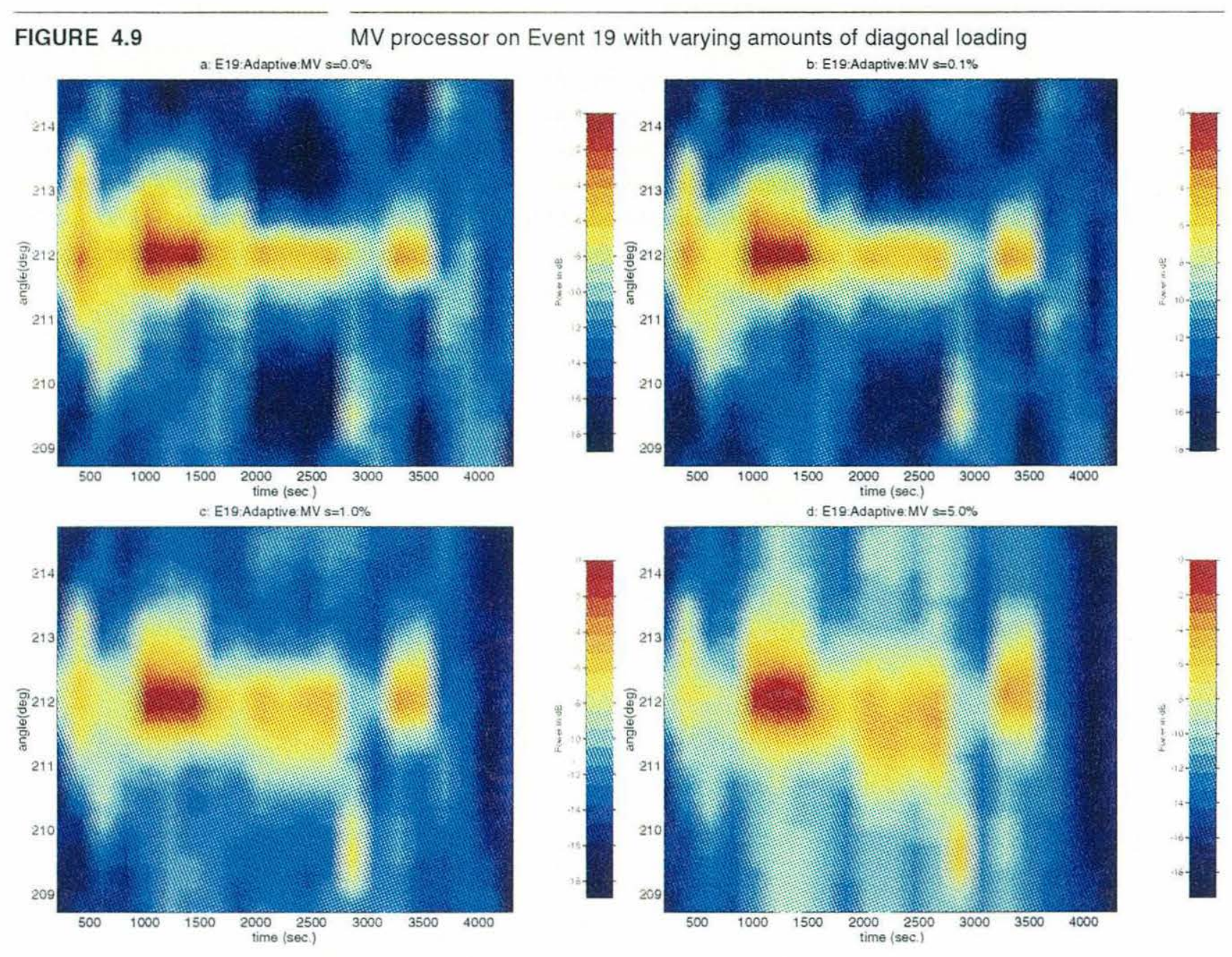

\subsubsection{MV and MUSIC processor results}

Adaptive processing is used to zoom in on the peak of the signal. For each event six plots are shown on the following pages. As discussed in chapter 3, information can be extracted from the eigenvalue magnitudes. The eigenvalue magnitudes are time varying so the first plot shows the power in each eigenvalue as a function of time. Below these plots the effective number of degrees of freedom of the sample covariance estimate is plotted. This is calculated by the squared sum of the eigenvalues divided by the sum of the squares:

$$
\frac{\left\langle\sum_{\mathrm{i}=1}^{\mathrm{N}} \lambda_{\mathrm{i}}\right\rangle^{2}}{\sum_{\mathrm{i}=1}^{\mathrm{N}} \lambda_{\mathrm{i}}^{2}} .
$$

If only one signal were present agianst a low noise background this would approach unity. If no signals are present, and only a white noise background is present this quantity would approach the number of array elements assuming an adequate number of snapshots. 
The second plot shows the results of conventional processing as a reference using the CHRA array with specifications as in section 3.2. The third plot shows the results of adaptive MV processing using the AHRA array. Time windows are $410 \mathrm{~s}$. as previously noted, and 32 channels have been summed in subarrays of four each to create the eight output channels. The fourth through sixth plots show the results of the MUSIC processor with an increasing number of eigenvalues. Each time a new eigenvalue-eigenvector is included it adds a uncorrelated signal or noise element. For instance, if a second signal were to appear next to the first on the second eigenvalue plot, and the third eigenvalue plot only added unstructured noise, this is an indicator of two multipath. 


\section{FIGURE 4.10}
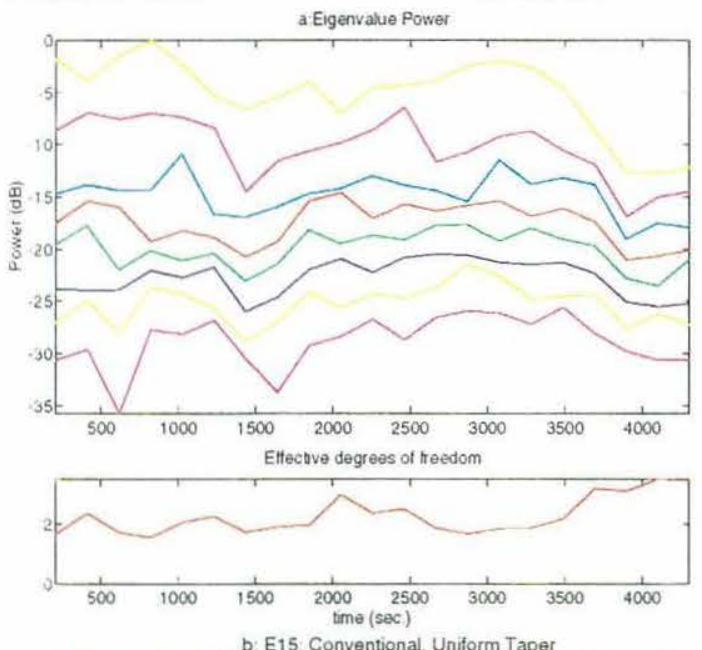

b. E15: Conventional, Uniform Tape

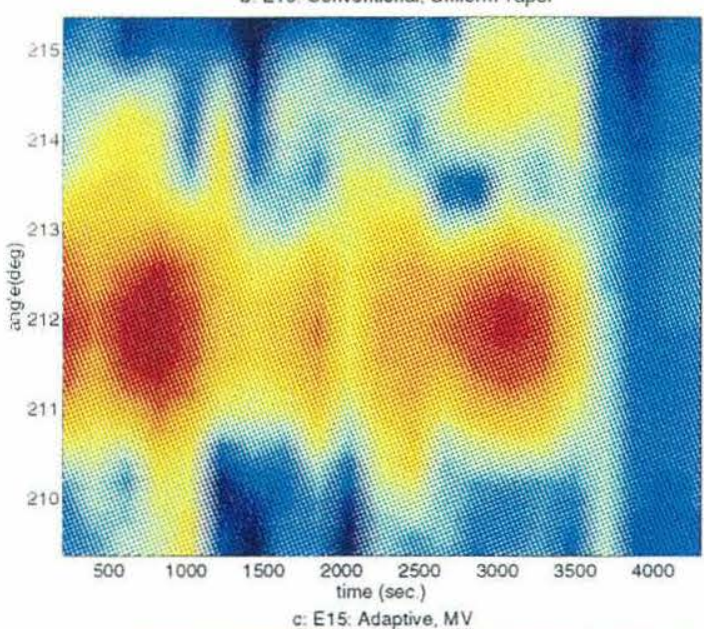

C: E15. Adaptive, MV

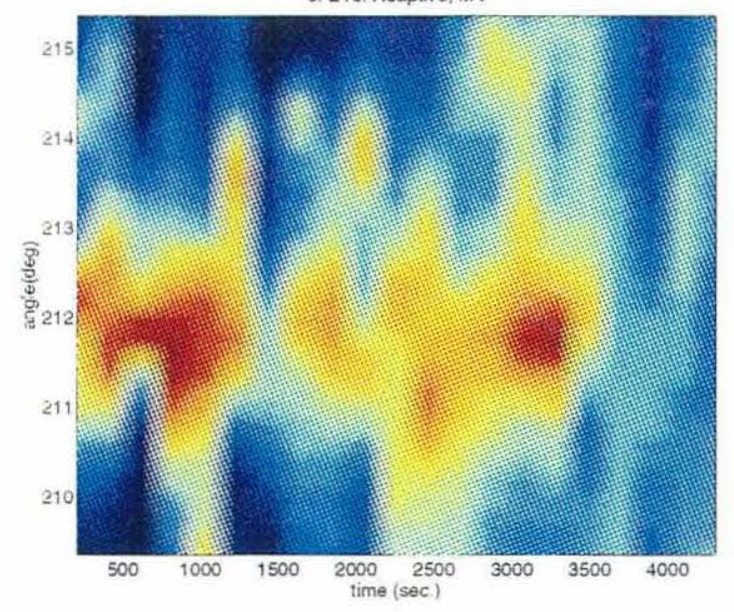

d. E15: MUSIC 1 eval
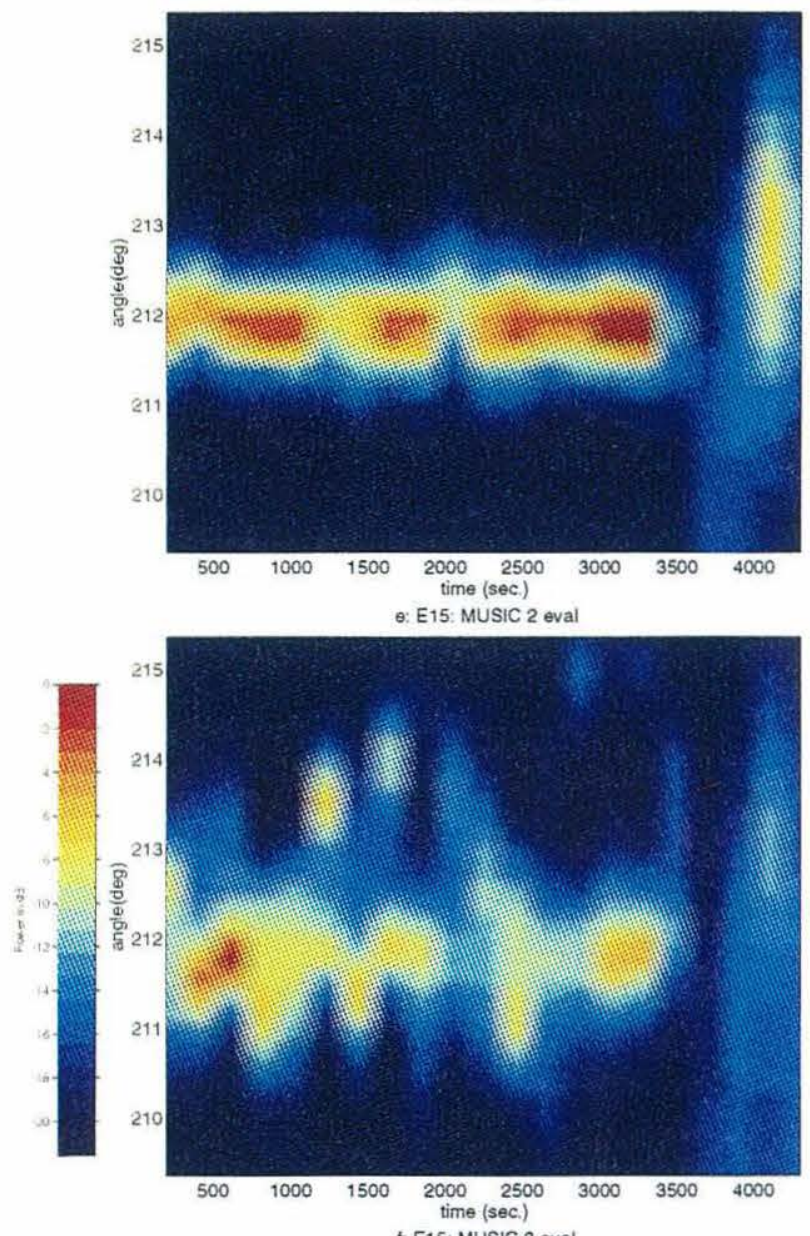

+ E15: MUSIC 3 eval

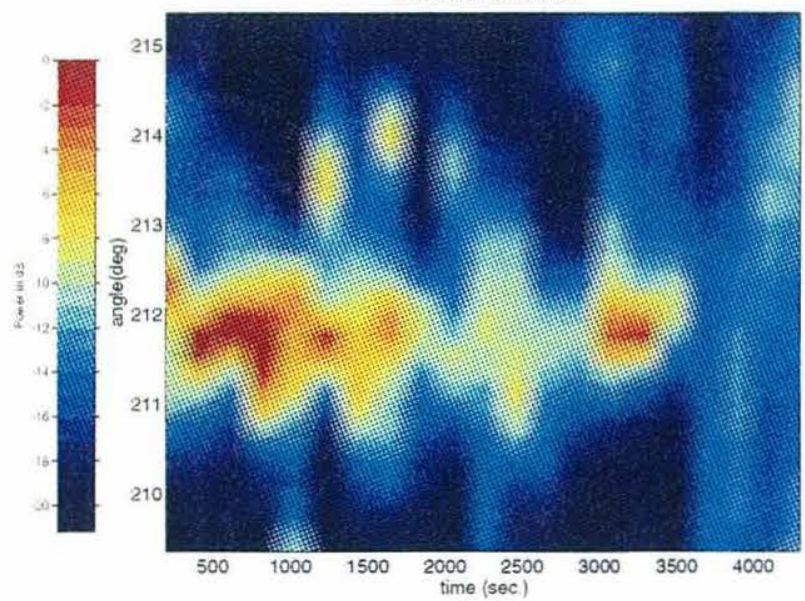


FIGURE 4.11
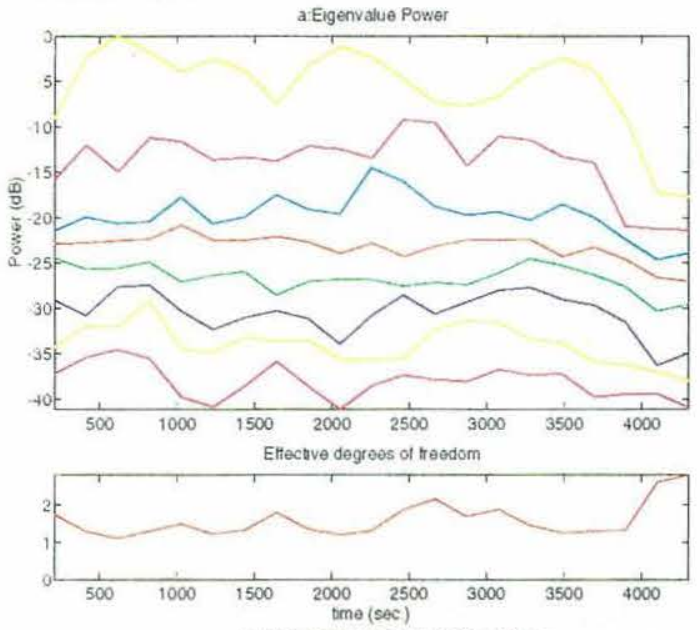

b. E18. Conventional, Uniform Taper
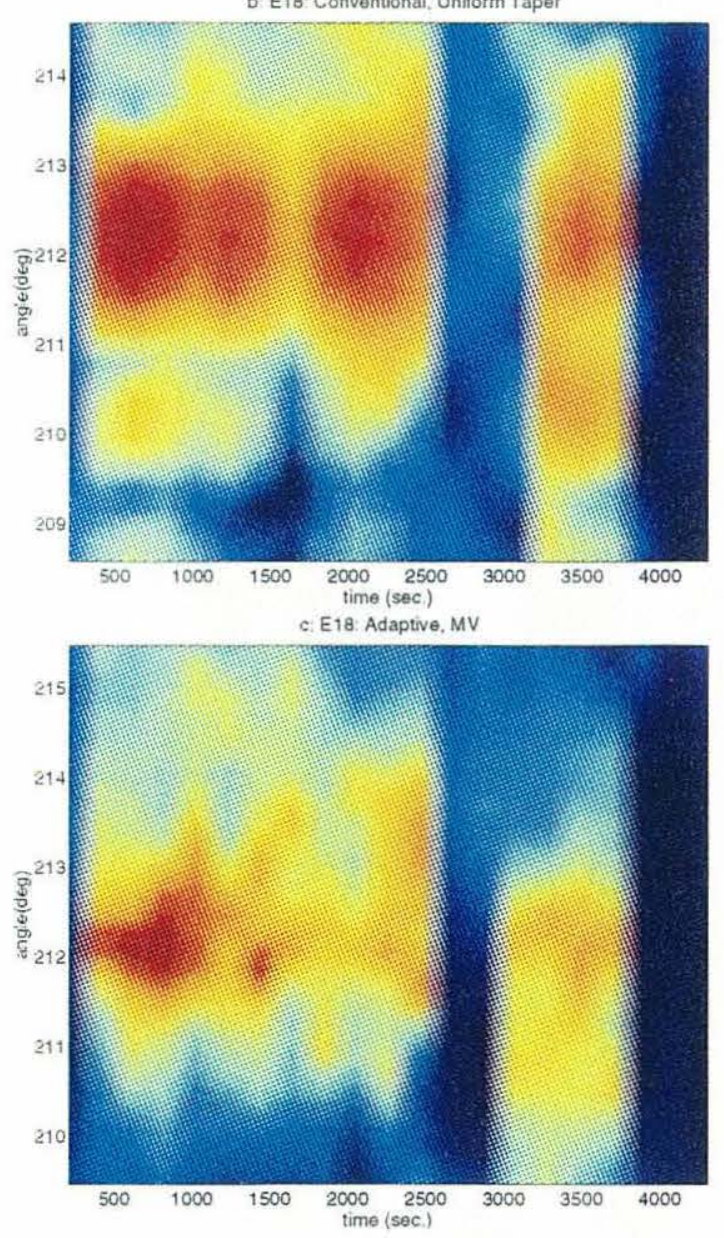

d. E18: MUSIC 1 eval
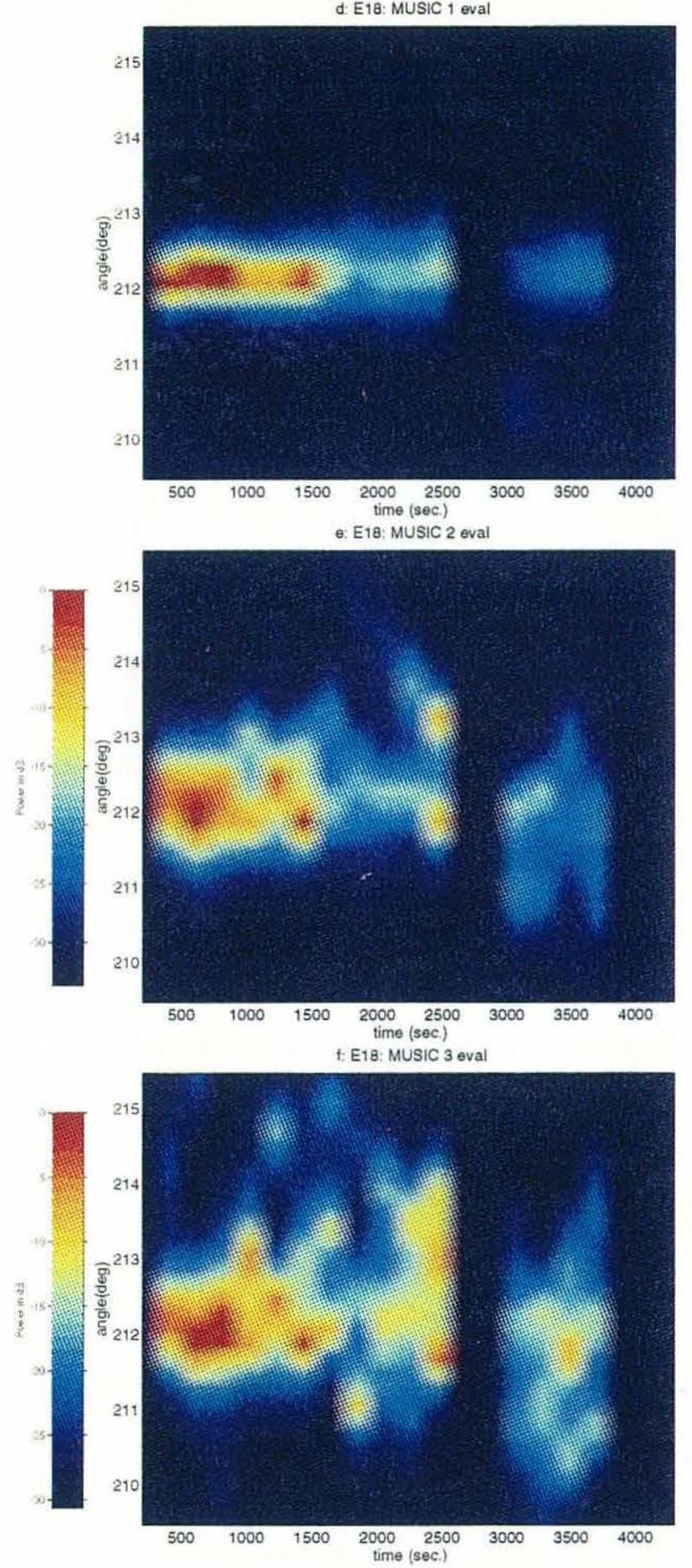
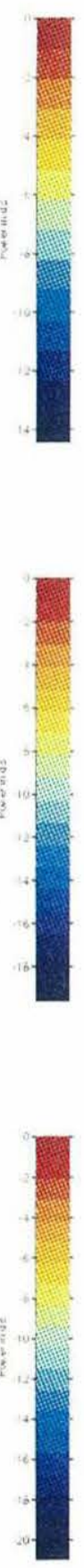
FIGURE 4.12
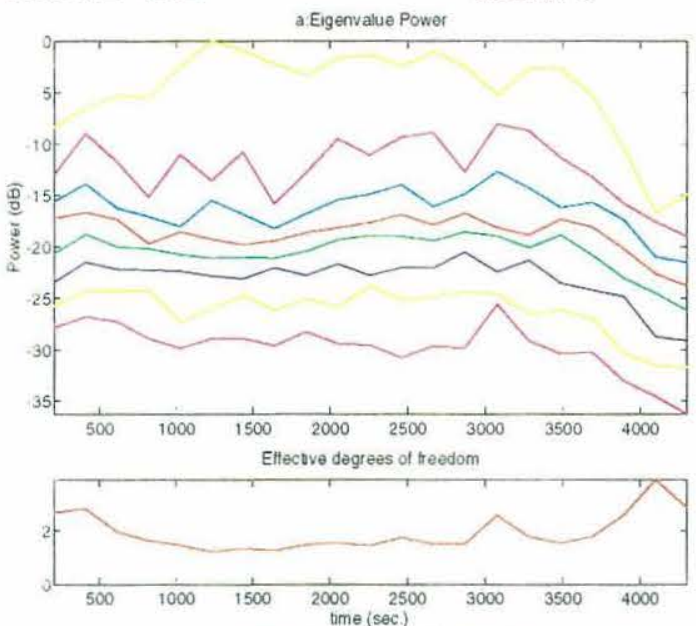

b. E19 Conventional. Uniform Taper
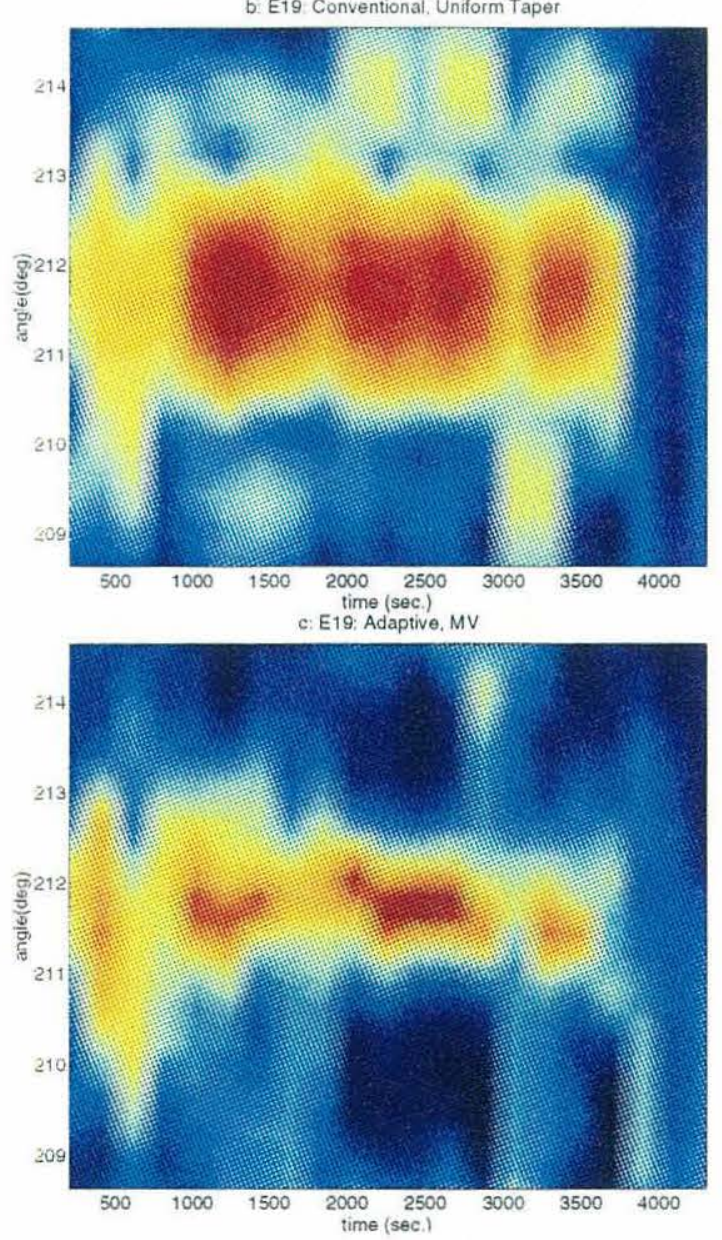

d: E19: MUSIC 1 eval

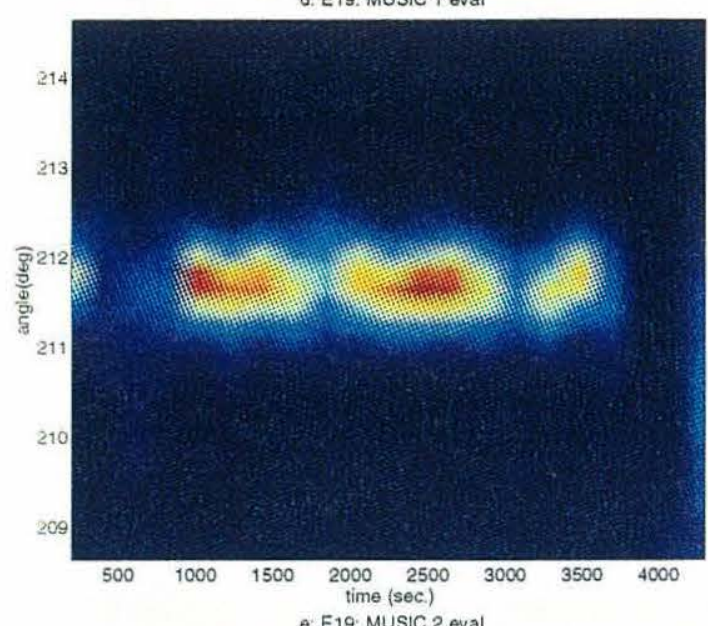

0. MUSIC 2 eva
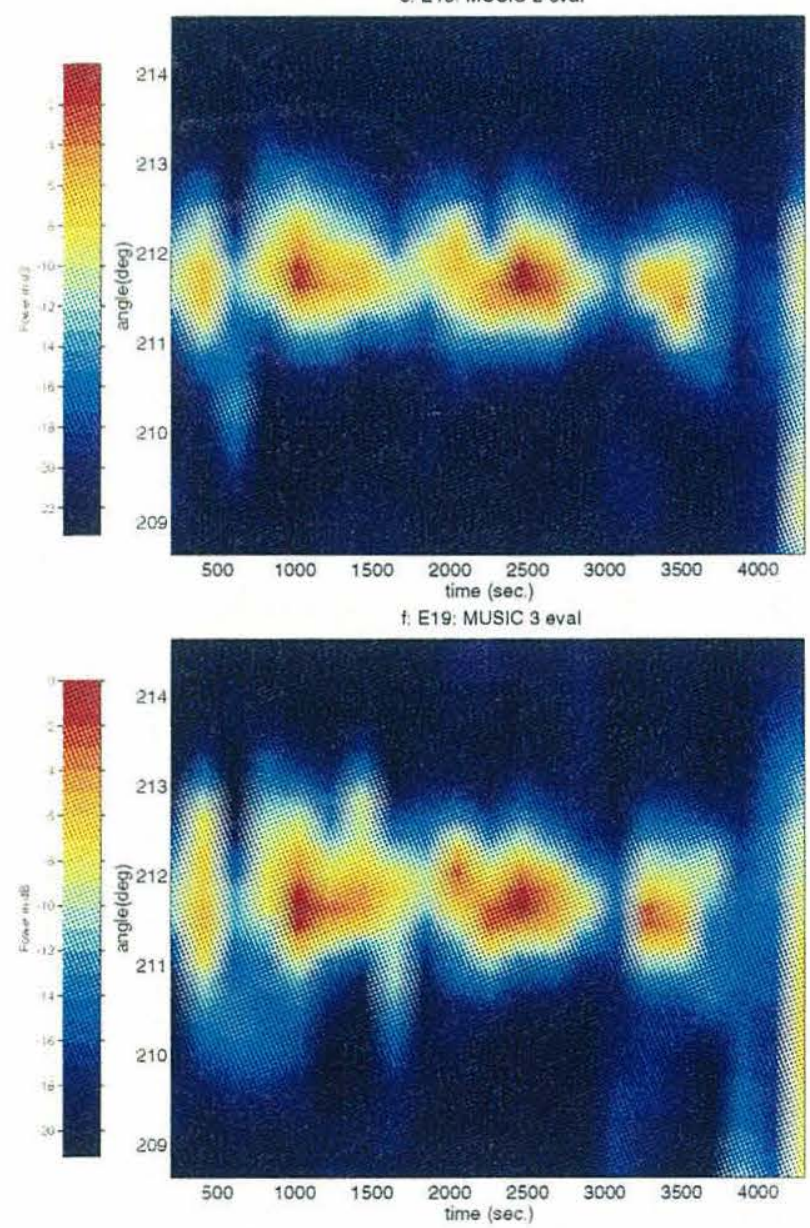


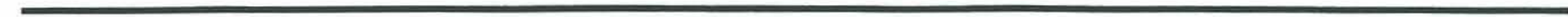


FIGURE 4.13
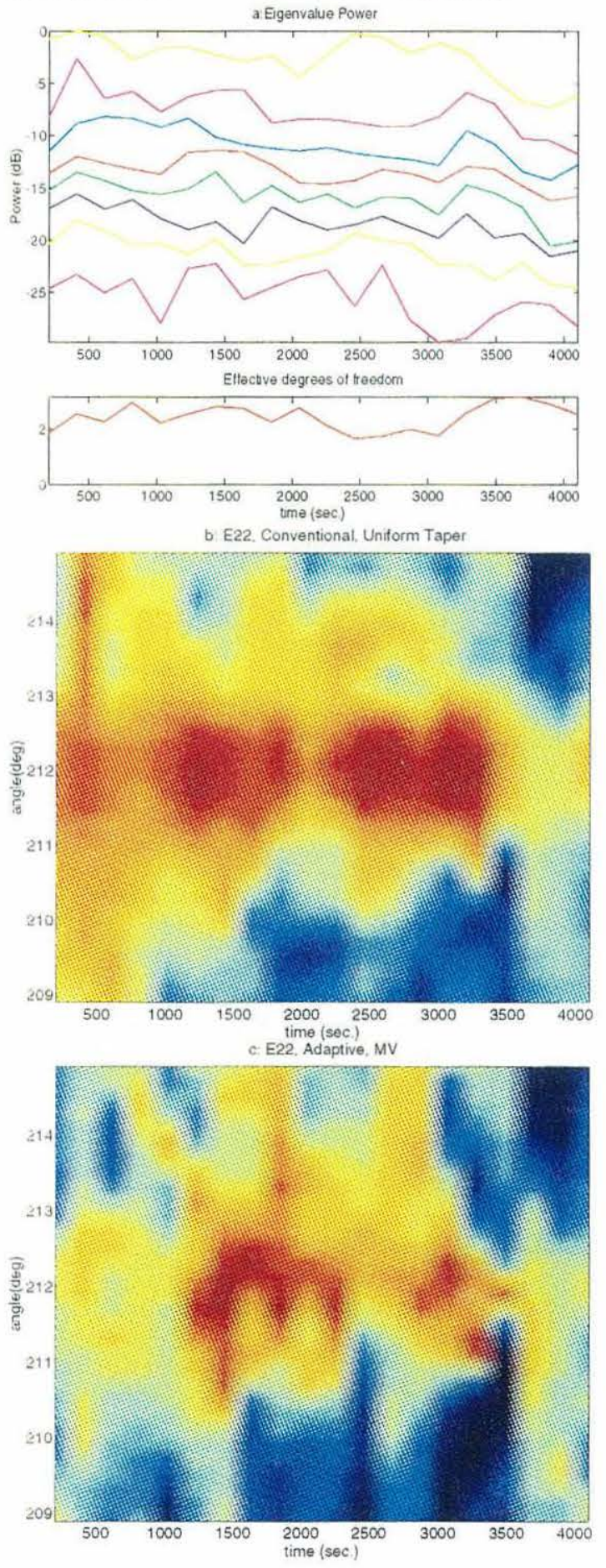

d. E22, MUSIC 1 eval

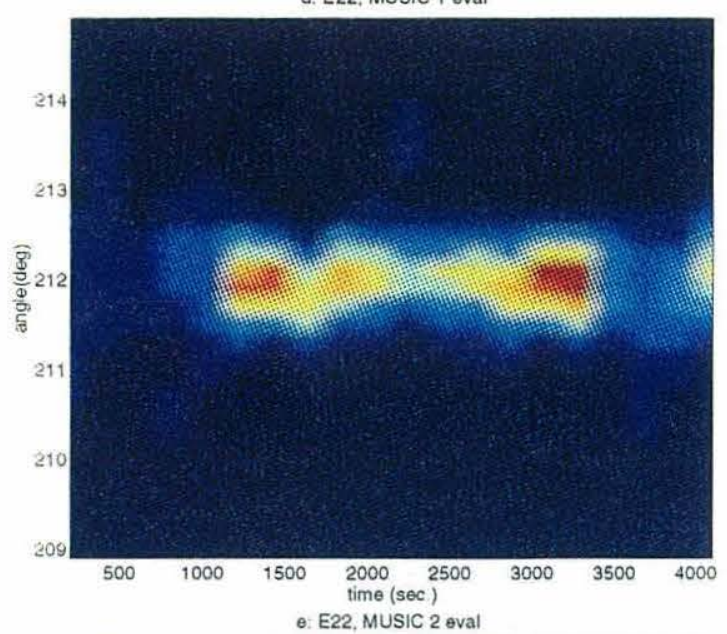

e: E22, MUSIC 2 eval
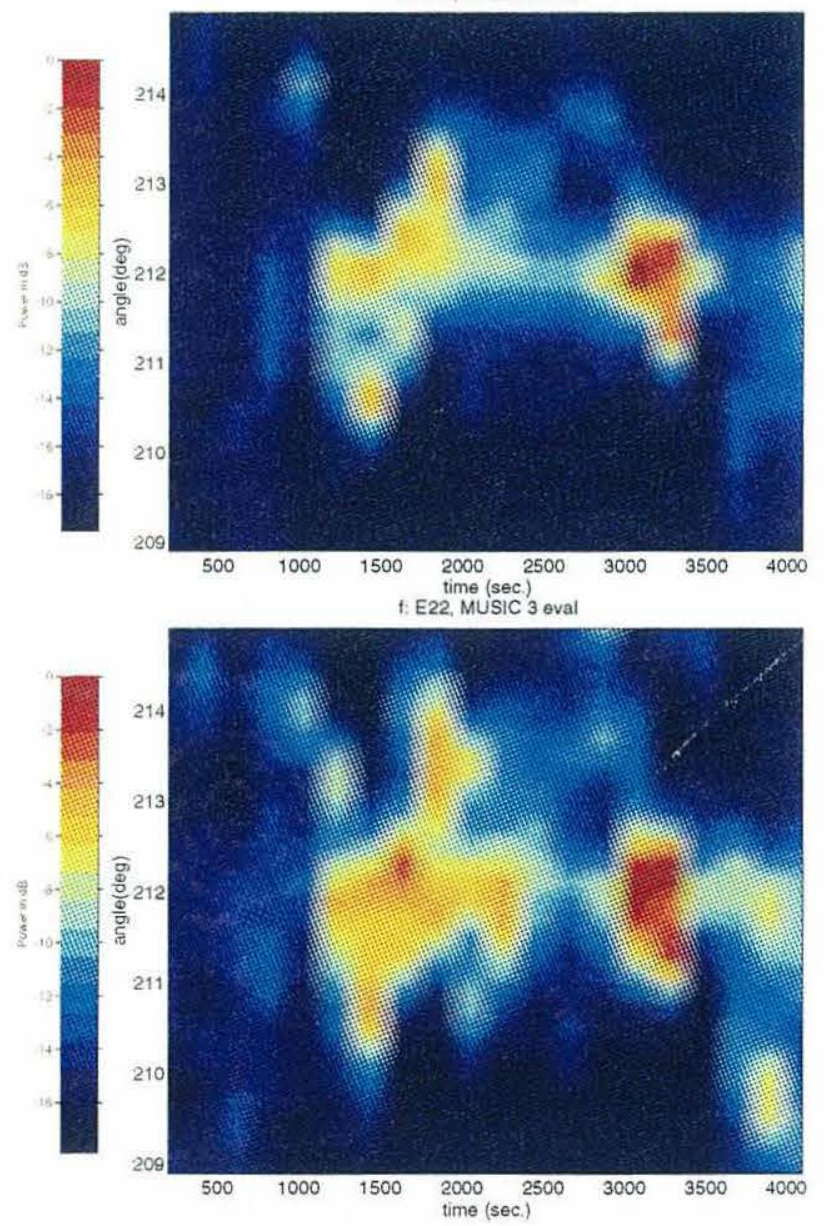
$\longrightarrow$ 
FIGURE 4.14
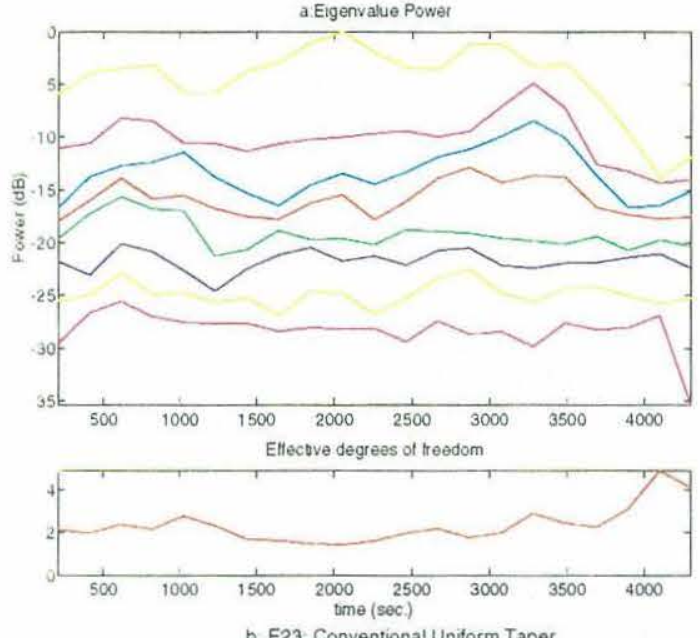

b. E23: Conventional Uniform Taper
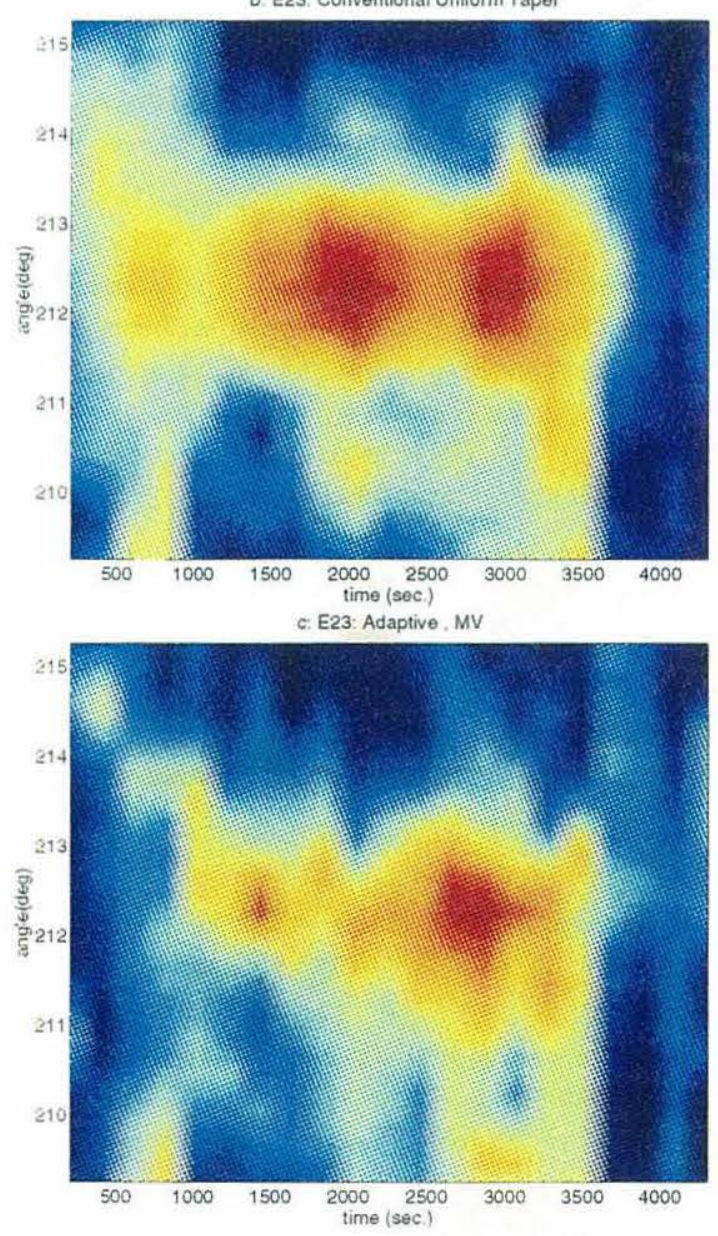

d. E23: MUSIC 1 eval
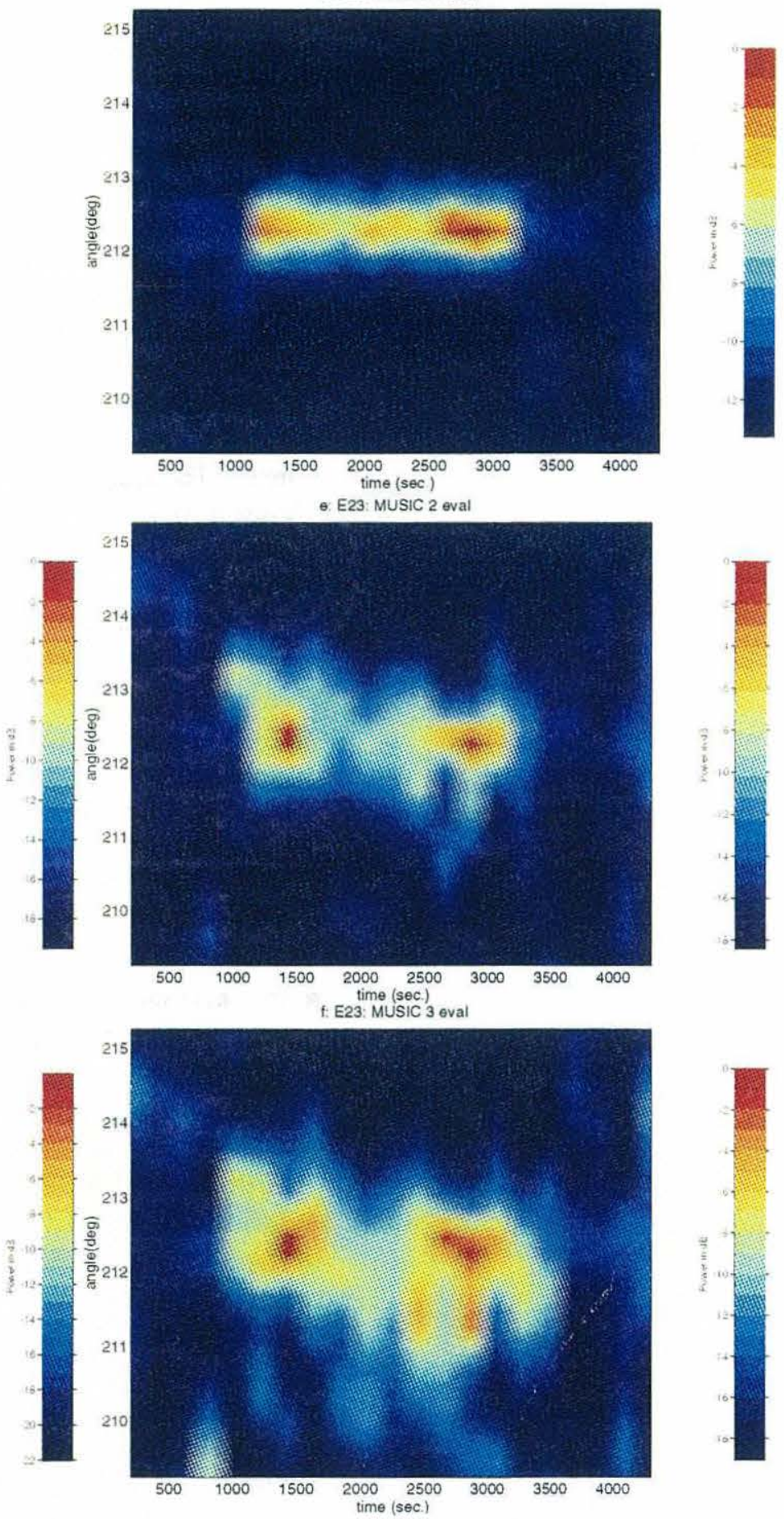


\subsubsection{Discussion}

a. Eigenvalue Spectrums

- The temporal fluctuations of the power level carried by the first eigenvalue are consistent with the temporal fluctuations of the power response from the conventional beamformer in all events. The power levels in the second through fourth eigenvalues fall off at the end of the signal reception at approximately $3600 \mathrm{sec}-$ onds. In all events except 22 there is also a fall off in power of the fifth and sixth eigenvalues at the end of the signal.

- Examining the relative power levels between eigenvalue does not clearly distinguish the transition between the signal space and the noise space in these eigenvalue spectrum plots. The first eigenvalue has a large step to the next lower eigenvalue consistently in all events, but not at all times during the reception. On events 15 and 18 the second eigenvalue also has a large step to the next lower eigenvalue at certain times.

- On all events the fluctuations in the middle eigenvalues follow similar temporal trends. The actual number of eigenvalues that show this similarity varies from event to event, but encompasses the range from the third to the seventh eigenvalue.

- The effective number of degrees of freedom approaches unity when the signal is at its maximum power and increases with decreasing signal to noise ratio. This is evidence for one dominant arrival.

b. Conventional Beamformer

- The temporal power fluctuations from the signal direction are independent from event to event. The most significant feature of the conventional beamformer response is there appears to be only one arrival direction present. A second feature of conventional beamformer is the spreading of the power in angle. This is most noticeable at the beginning of event 22 . These areas of spreading are well correlated in time when the turning and curvature indices are high.

c. MV beamformer

- The angle spreading that is visible in the conventional beamformer is visible as spreading and as multiple peaks in the higher resolution MV beamformer. This is consistent with the sensitivity analysis to array curvature.

- The temporal power fluctuations from the MV beamformer follow similar trends as the conventional beamformer, and the MV processor shows similar signal to noise levels as the conventional processor.

d. MUSIC beamformer

m The MUSIC response due to the first eigenvalue is stable in direction and narrow in resolution. Its temporal power fluctuations are consistent with those of the conventional beamformer.

- In the events 15,18 , and 22 , with significant signal spreading multiple peaks occur with the addition of second and third eigenvalue responses. The response due to three eigenvalues contains most of the features of the MV processor.

- The angular width of the MUSIC response implies that it is difficult to resolve multipath arrivals seperated by less than $0.5^{\circ}$ if such arrivals exsist. 


\subsection{Beamforming conclusions}

1. There is one dominant arrival as shown by the first Eigenvalue response of the MUSIC beamformer and the center angle peak of the conventional beamformer at a mean angle of $212^{\circ} \mathrm{T}$.

2. There is no stable secondary horizontal mutlipath which would be visible as a parallel arrival to the first.

3. There are uncorrelated signal elements arriving on the array as seen by the eigenvalue structure. The fall off in power of the lower order eigenvalues at the end of the transmissions indicates that these signals are not ambient noise. Many factors could contribute to this effect. As shown in chapter 3, array curvature can cause poor performance of the beamformer, but does not alter the eigenvalue spectrum considerably. The time windows for forming the covariance matrices were 410 seconds long, so the uncorrelated signals could be caused by loss of coherence over this time interval or possibly by the spatial structure of the signal. The most likely explanation of this is that fluctuations of the doppler shift about its mean causes the one arrival to decorrelate over the time window. One way to possibly overcome this is to demodulate the doppler shift more accurately. This requires not only demodulating the mean, but also the fluctuations about the mean shift. With the low SNR on one hydrophone used for estimating the doppler shift it is not possible to track the fluctuations in the doppler shift. If the beamformer is used as a spatial filter it may be possible to achieve high enough SNR to track the fluctuations.

The two most important results of this thesis are there is no evidence of any horizontal multipath, and the mean arrival angle is estimated to be from $212^{\circ} \mathrm{T} \pm 1.5^{\circ}$.

It is also important to note at this stage that it is not proven that horizontal multipath does not exist. It is only proven that multipath is undetectable on this data set with the methods of analysis that are employed. As mentioned in the introduction McDonald et al. [2] predicted two eigenray bundles reaching the receiving station. The expected arrival at $214^{\circ} \mathrm{T}$ has been detected at a slightly lower angle. The second arrival was predicted at $219^{\circ} \mathrm{T}$ with power $40 \mathrm{~dB}$ lower than the first. If this signal exists it can not be detected with the available arrays and processing techniques given the SNR. Within each eigenray bundle the predicted individual vertical modes of propagation had horizontal arrival angles differing from a tenth to half a degree. This small separation is unresolved even with the adaptive processors.

Time compression results will yield an interesting second view of the horizontal multipath problem. If arrival peaks are separated by time intervals larger than can be explained by vertical mode structure, then further work on this issue is warranted. Fixed arrays with known geometries as planned in the Acoustic Thermography of Ocean Climate (ATOC) experiments may also provide information on horizontal multipath. The ATOC experiments will be on a basin scale as opposed to the global scale of the HIFT. 
[1] G.J. Heard. Personal Communication

[2] B. E. McDonald, M. D. Collins, W. A. Kuperman and K. D. Heaney (1993),"Comparison of Data and Model Predictions For Heard Island Acoustic Transmissions," J. Acoust. Soc. Am. (HIFT special issue.) 